POLLYANA GIL CUNHA

ANÁLISE DINÂMICA DE PONTES FERROVIÁRIAS: UMA METODOLOGIA SIMPLIFICADA 
POLLYANA GIL CUNHA

\section{ANÁLISE DINÂMICA DE PONTES FERROVIÁRIAS: UMA METODOLOGIA SIMPLIFICADA}

Dissertação apresentada à Escola Politécnica da Universidade de São Paulo para obtenção do título de Mestre em Engenharia.

Área de Concentração: Engenharia de Estruturas

Orientador: Prof. Dr. Carlos Eduardo de Nigro Mazzilli 
POLLYANA GIL CUNHA

\title{
ANÁLISE DINÂMICA DE PONTES FERROVIÁRIAS: UMA METODOLOGIA SIMPLIFICADA
}

\author{
Dissertação apresentada à Escola \\ Politécnica da Universidade de São Paulo \\ para obtenção do título de Mestre em \\ Engenharia.
}


AUTORIZO A REPRODUÇÃO E DIVULGAÇÃO TOTAL OU PARCIAL DESTE TRABALHO, POR QUALQUER MEIO CONVENCIONAL OU ELETRÔNICO, PARA FINS DE ESTUDO E PESQUISA, DESDE QUE CITADA A FONTE.

Este exemplar foi revisado e alterado em relação à versão original, sob responsabilidade única do autor e com a anuência de seu orientador.

São Paulo, 26 de abril de 2011.

Assinatura do autor

Assinatura do orientador

FICHA CATALOGRÁFICA

Cunha, Pollyana Gil

Análise dinâmica de pontes ferroviárias: uma metodologia simplificada / P.G. Cunha. -- ed.rev. -- São Paulo, 2011.

p.

Dissertação (Mestrado) - Escola Politécnica da Universidade de São Paulo. Departamento de Engenharia de Estruturas e Geotécnica.

1. Dinâmica das estruturas I. Universidade de São Paulo. Escola Politécnica. Departamento de Engenharia de Estruturas e Geotécnica II. t. 


\section{FICHA DE APROVAÇÃO}

Pollyana Gil Cunha

Análise dinâmica de pontes ferroviárias: uma metodologia simplificada.

Dissertação apresentada à Escola Politécnica da Universidade de São Paulo para obtenção do título de Mestre em Engenharia.

Área de Concentração: Engenharia de Estruturas.

Aprovado em:

\section{BANCA EXAMINADORA}

Prof. Dr.

Instituição: Assinatura:

Prof. Dr.

Instituição: Assinatura:

Prof. Dr. Instituição: Assinatura: 
Ao meu futuro esposo, por toda ajuda, incentivo e apoio. 


\section{AGRADECIMENTOS}

Agradeço em primeiro lugar a Deus, por me amparar nos momentos difíceis e me dar forças para superar as dificuldades.

Agradeço à Escola Politécnica, por me proporcionar conhecimento, espaço físico e as ferramentas necessárias para desenvolver este trabalho.

Ao Prof. Dr. Carlos Eduardo de Nigro Mazzilli por toda orientação, ensinamento, incentivo e amizade.

À minha família, que sempre me apoiou, incentivou e esteve ao meu lado.

Ao meu futuro esposo Carlos Eduardo, por toda paciência, ajuda, apoio e incentivo em todos os momentos de desenvolvimento deste trabalho.

Aos amigos da Themag Engenharia, que me dispuseram o tempo necessário para a realização deste trabalho.

Ao amigo Sérgio Cifú, pelos ensinamentos, paciência e incentivo.

À minha amiga Érika, que esteve presente em todos os momentos, me dando força e incentivo.

Às minhas amigas Carol e Bruna, que sempre se dispuseram a me ajudar. 
A paciência e a perseverança é um dos métodos mais eficientes que usamos para conquistar um objetivo.

(Michel Franklly) 


\section{RESUMO}

CUNHA, P.G. Análise dinâmica de pontes ferroviárias: uma metodologia simplificada, 2011. Dissertação (Mestrado) - Escola Politécnica, Universidade de São Paulo, 2011.

Este trabalho trata do problema das vibrações induzidas em estruturas de concreto de pontes ferroviárias, produzidas pela passagem da composição de um TUE (Trem Unidade Elétrico) típico. Partiu-se de um modelo do veículo com nove graus de liberdade, referentes aos deslocamentos verticais e rotações em torno dos eixos longitudinal e transversal, introduzindo-se os graus de liberdade de deslocamentos transversais horizontais e rotações em torno do eixo vertical. Foram calculados os carregamentos provenientes do trem modelado sobre tabuleiro rígido indeslocável, considerando as irregularidades geométricas apresentadas nos trilhos e nas rodas, sendo diferenciada para cada linha de trilho. Estes carregamentos foram condensados estaticamente e aplicados em um modelo estrutural simplificado (unifilar) de uma ponte de seção celular, visando a identificar a resposta dinâmica proveniente das irregularidades geométricas. Finalmente, avalia-se a amplificação dinâmica, a qual é comparada com o valor do coeficiente de impacto prescrito na norma brasileira para projeto de pontes ferroviárias.

Palavra Chave: Dinâmica das Estruturas, Pontes Ferroviárias, Irregularidades Geométricas. 


\begin{abstract}
CUNHA, P.G. Dynamic analysis of railway bridges: a simplified methodology, 2011. Dissertation (Master of Engineering) - Escola Politécnica, Universidade de São Paulo, 2011.

This dissertation discusses the problem of induced vibrations in concrete structures of railway bridges, produced by the passing of a typical composition of electric urban trains. It started from a vehicle model with nine degree-of-freedom, related to vertical displacements and rotations around the longitudinal and transverse axes, introducing the degrees-of-freedom of horizontal transverse displacements and rotations around the vertical axis. The train's loads applied to the bridge deck were evaluated, assuming initially a rigid structure under the board, considering the geometric irregularities present in rails and wheels and different for each rail line. These loads were statically reduced to the vehicle center of mass and applied to a simplified bridge structural model, aiming at identifying the dynamic response due to geometric irregularities. Finally the dynamic amplification is evaluated and compared to the value of the impact coefficient prescribed by the Brazilian Standards for design of railway bridges.
\end{abstract}

Keywords: Dynamics of Structures, Railway Bridges, Geometric Irregularities. 


\section{LISTA DE FIGURAS}

Figura 1: Ponte da São Paulo Railway - Trecho da Serra [29] .................................16

Figura 2: Graus de liberdade considerados neste estudo ...........................................20

Figura 3: Trem urbano em São Paulo (CPTM) [23] …….........................................24

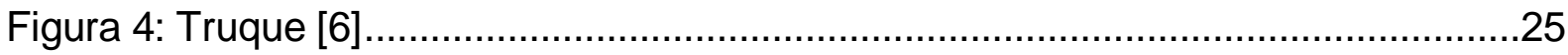

Figura 5: Esquema de uma típica locomotiva diesel-elétrica [22] ................................26

Figura 6: Locomotivas diesel-elétricas utilizadas no Brasil [22] .................................26

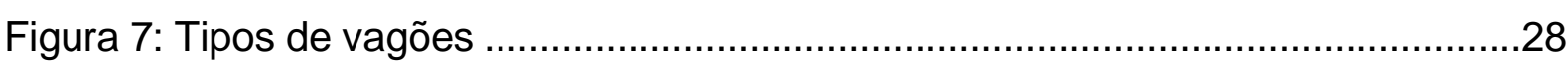

Figura 8: Características geométricas e cargas dos trens-tipo ..................................29

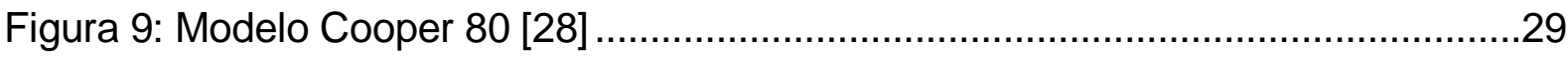

Figura 10: Diagrama do Coeficiente de impacto x Comprimento do vão da ponte .......30

Figura 11: Modelo dinâmico do carro ....................................................................33

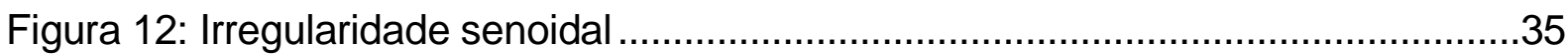

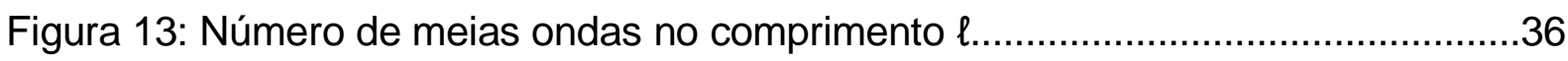

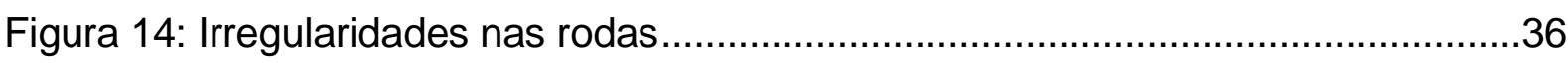

Figura 15: Detalhe da roda com achatamento ……...............................................

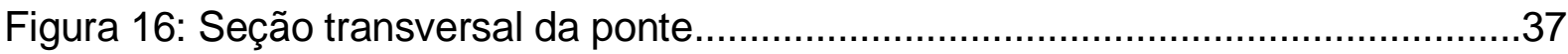

Figura 17: Redução de esforços de interação para o centro de gravidade ...................52

Figura 18: Modelo unifilar da ponte ......................................................................54

Figura 19: Modelo veicular utilizando o ADINA.......................................................55

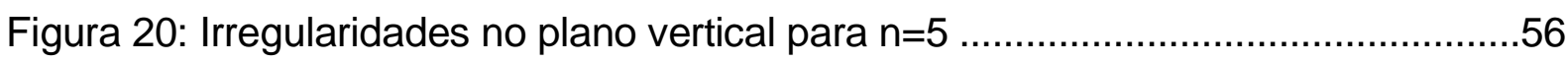

Figura 21: Irregularidades no plano horizontal transversal para $n=5 \ldots \ldots \ldots \ldots \ldots \ldots \ldots \ldots \ldots . . .56$

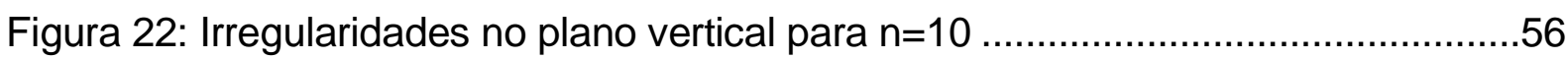

Figura 23: Irregularidades no plano horizontal transversal para $n=10 \ldots \ldots \ldots \ldots \ldots \ldots \ldots \ldots . . .57$

Figura 24: Irregularidades no plano vertical para $n=20 \ldots \ldots \ldots \ldots \ldots \ldots \ldots \ldots \ldots \ldots \ldots \ldots \ldots \ldots . . .57$

Figura 25: Irregularidades no plano horizontal transversal para $n=20 \ldots \ldots \ldots \ldots \ldots \ldots \ldots \ldots . .57$

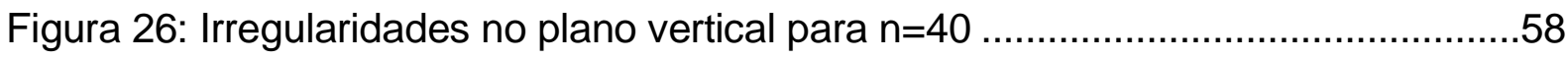

Figura 27: Irregularidades no plano horizontal transversal para $n=40 \ldots \ldots \ldots \ldots \ldots \ldots \ldots \ldots . . .58$

Figura 28: Modos de vibração do carro .................................................................61

Figura 29: Modelo de casca utilizado para a ponte .......................................................

Figura 30: Modos de vibração da ponte .................................................................

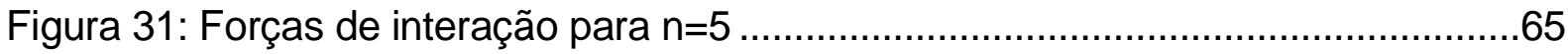

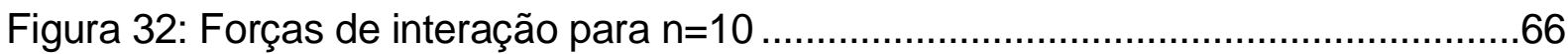




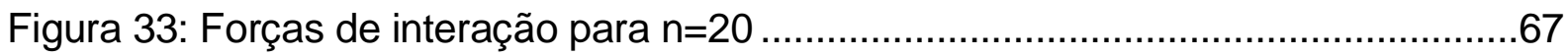

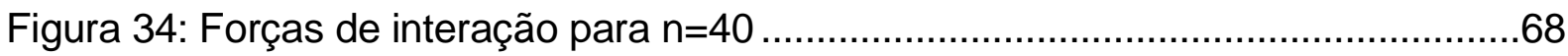

Figura 35: Resultados - análise quase-estática ...................................................

Figura 36: Linha de influência de momento fletor ....................................................71

Figura 37: Situação em que um segundo carro entra na ponte ..................................71

Figura 38: Situação com dois carros sobre a ponte..................................................

Figura 39: Detalhes dos digramas de momentos fletores ..........................................72

Figura 40: Redução na reação vertical máxima......................................................73

Figura 41: Esquema de carregamento utilizado na análise dinâmica............................74

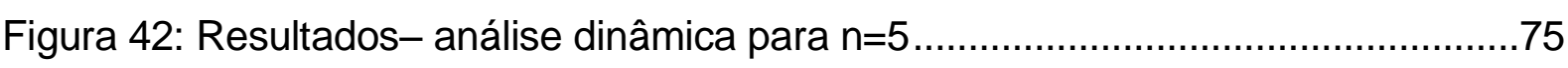

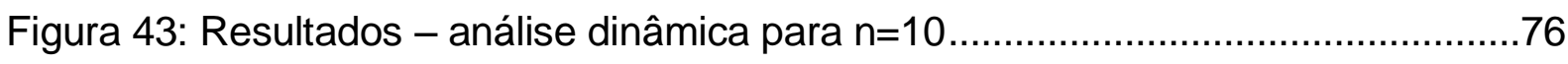

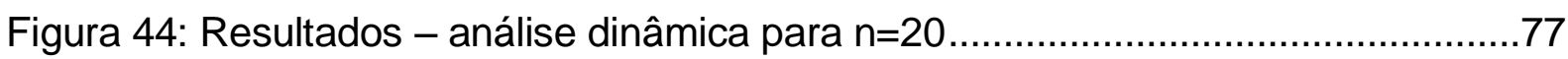

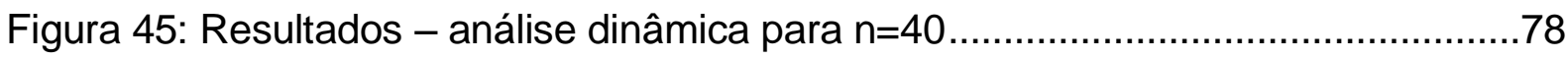

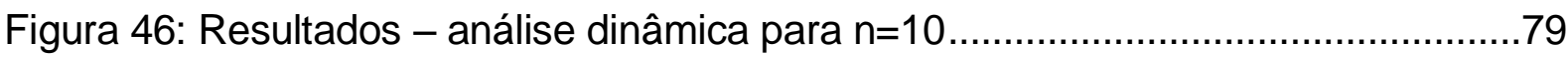




\section{LISTA DE TABELAS}

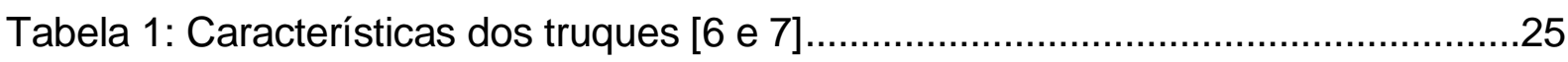

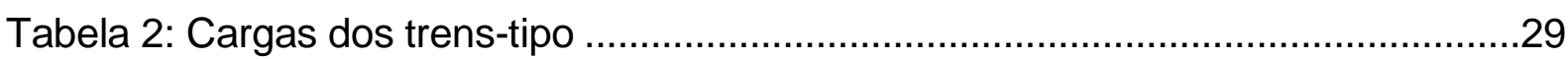

Tabela 3: Parâmetros mecânicos e dinâmicos do carro.................................................34

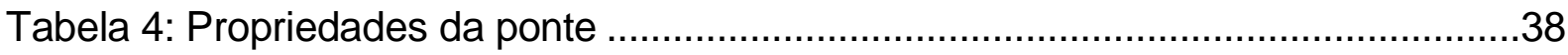

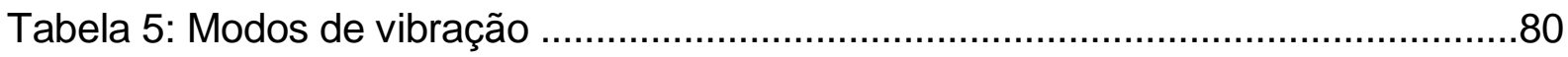

Tabela 6: Valores máximos obtidos em cada análise $[\mathrm{kN} . \mathrm{m}, \mathrm{kN}$ e mm] .....................81

Tabela 7: Acréscimo da resposta referente ao coeficiente de impacto e análise

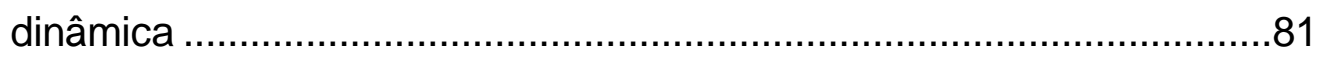

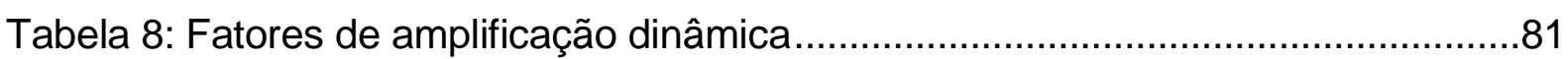

Tabela 9: Relação entre esforços da análise dinâmica e os da análise quase-

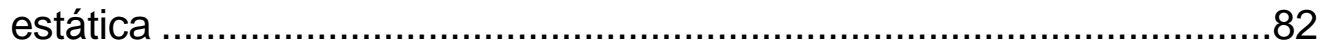

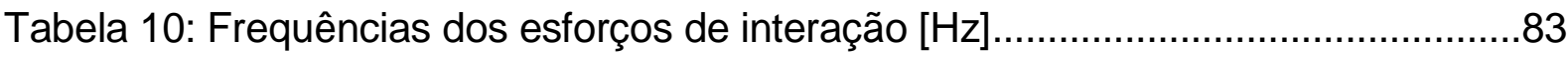




\section{SUMÁRIO}

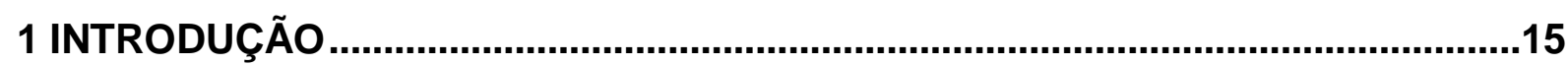

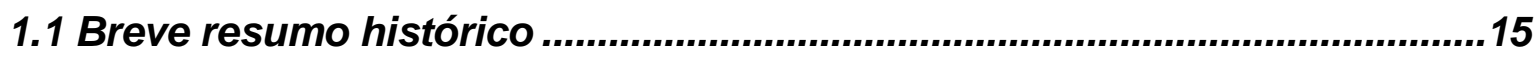

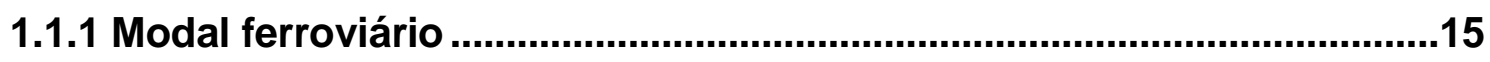

1.1.2 Efeito dinâmico em estruturas de pontes...............................................17

1.2 Objetivos e justificativas ..................................................................................21

1.3 Metodologia............................................................................................................22

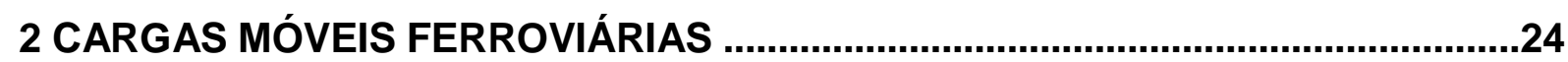

2.1 Descrição dos tipos de carros ou vagões ferroviários utilizados no Brasil

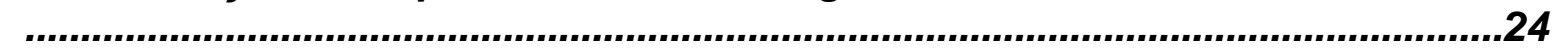

2.2 Modelo de Carga Móvel Prescrito na NBR-7189 _.......................................28

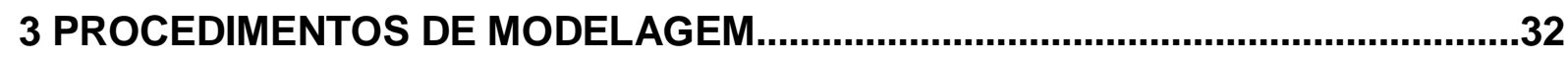

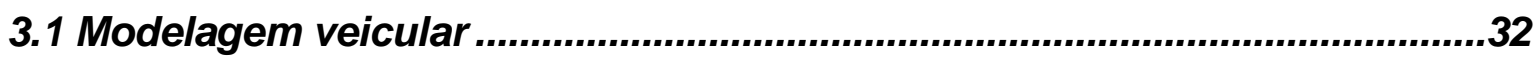

3.2 Irregularidades geométricas nos trilhos e nas rodas...................................34

3.3 Modelagem estrutural ....................................................................................37

4 FORÇAS DE INTERAÇÃO E EQUAÇÕES DE MOVIMENTO ……........................39

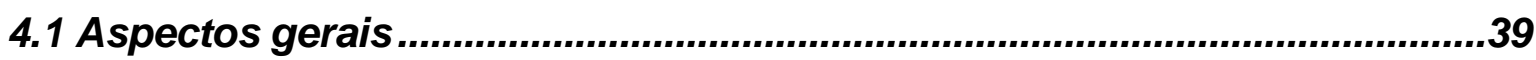

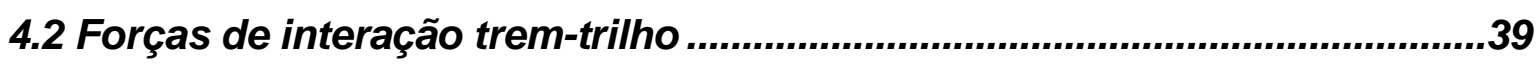

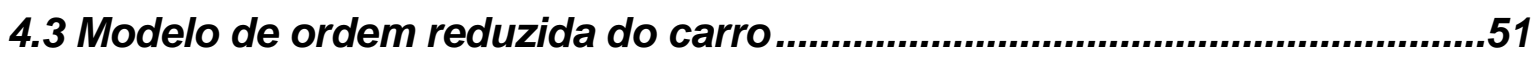

4.4 Aplicação dos esforços de contato no modelo estrutural.............................53

5 ESTUDO DE CASO: FORÇAS NO CONTATO RODA-TRILHO E ESFORÇOS MÓVEIS ESTATICAMENTE REDUZIDOS AO CENTRO DE GRAVIDADE DO CARRO

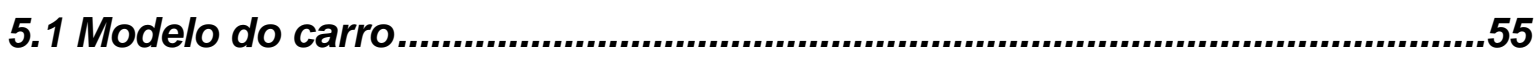

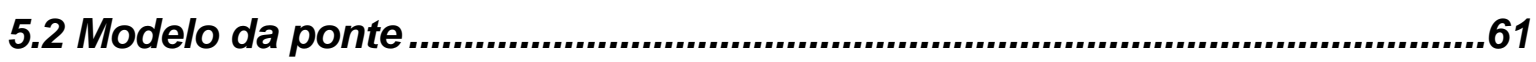

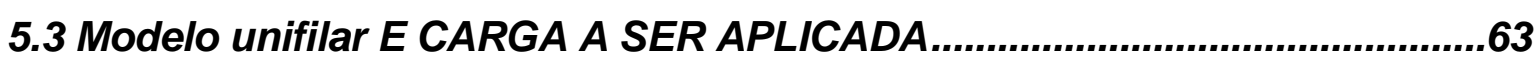

6 ESTUDO DE CASO: RESPOSTA DINÂMICA ESTRUTURAL EM DECORRÊNCIA DA PASSAGEM DO TREM 
8 SUGESTÕES PARA TRABALHOS FUTUROS

.85

9 REFERÊNCIAS BIBLIOGRÁFICAS. 


\section{INTRODUÇÃO}

\subsection{BREVE RESUMO HISTÓRICO}

\subsubsection{Modal ferroviário}

$O$ processo de implantação da estrada de ferro como meio de transporte terrestre ocorreu entre o fim do século XVIII e início do século XIX. A origem das estradas de ferro está ligada ao trabalho nas minas de carvão da Inglaterra.

Muitos experimentos surgiram através da força expansiva do vapor d'água e depois da máquina a vapor, em 1770, por James Watt. Várias tentativas foram feitas até que se tornasse possível a construção de um veículo que pudesse se locomover em uma estrada e que fosse movido pela força de expansão do vapor d'água. Muitas dessas tentativas não tiveram êxito. Somente em julho de 1814, George Stephenson finalmente conseguiu movimentar alguns vagões com sua máquina, utilizando o vapor d'água. Essa primeira locomotiva recebeu o nome de "The Rocket".

Até aquele momento haviam sido realizados apenas experimentos, porém em 25 de setembro de 1825 foi feita a primeira viagem, em caráter inaugural, no interior da Inglaterra (Stockton \& Darlington Railway). A partir desta data ficou consagrada definitivamente a estrada de ferro como meio de transporte terrestre.

No Brasil, a primeira tentativa, sem sucesso, para implantação de uma estrada de ferro ocorreu em 1835, após incentivo do Regente Diogo Antônio Feijó, que disponibilizou recursos para a sua construção e exploração, que possibilitaria ligações entre a cidade do Rio de Janeiro e as capitais de Minas Gerais, Rio Grande do Sul e Bahia. Outras tentativas ocorreram igualmente sem sucesso.

A produção agrícola, mais precisamente o café, precisava neste período de um meio de transporte mais eficiente e que reduzisse os gastos excessivos. É de 1852 , durante o Segundo Império, a lei número 641 que autorizou a construção de estradas de ferro no Brasil. A partir de então, muitas outras estradas surgiram.

Em 1852, o Barão de Mauá construiu a ligação entre o Porto de Mauá (interior da Baía de Guanabara) e a raiz da Serra (Petrópolis). A primeira seção, de $145 \mathrm{~km}$, foi inaugurada em 30 de abril de 1854 por D. Pedro II. O trem foi tracionado pela locomotiva "Baronesa", nome este dado em homenagem à esposa do Barão de Mauá. 
No ano de 1858 inaugurou-se a segunda ferrovia brasileira, que ligava Recife e São Francisco e ainda, a Estrada de Ferro D. Pedro II, que em 1889 tornou-se a Estrada de Ferro Central do Brasil.

Em 1860, iniciaram-se os trabalhos de construção da estrada de ferro que ligava Santos à Jundiaí. O transporte de cargas até então era feito por tropas de muares, extremamente precário e limitado, o que inibia o desenvolvimento tanto do Planalto quanto da Baixada. A distância entre as cidades contava ainda com uma declividade abrupta da Serra, o que tornava o custo da obra altíssimo. Esta ferrovia, inaugurada em 1867 e nomeada São Paulo Railway, foi realizada com recursos ingleses, ficando sob seu monopólio até 1946. A partir deste ano, foi tomada pelo governo brasileiro, sendo transformada em 1947 na Estrada de Ferro Santos-Jundiaí (EFSJ).

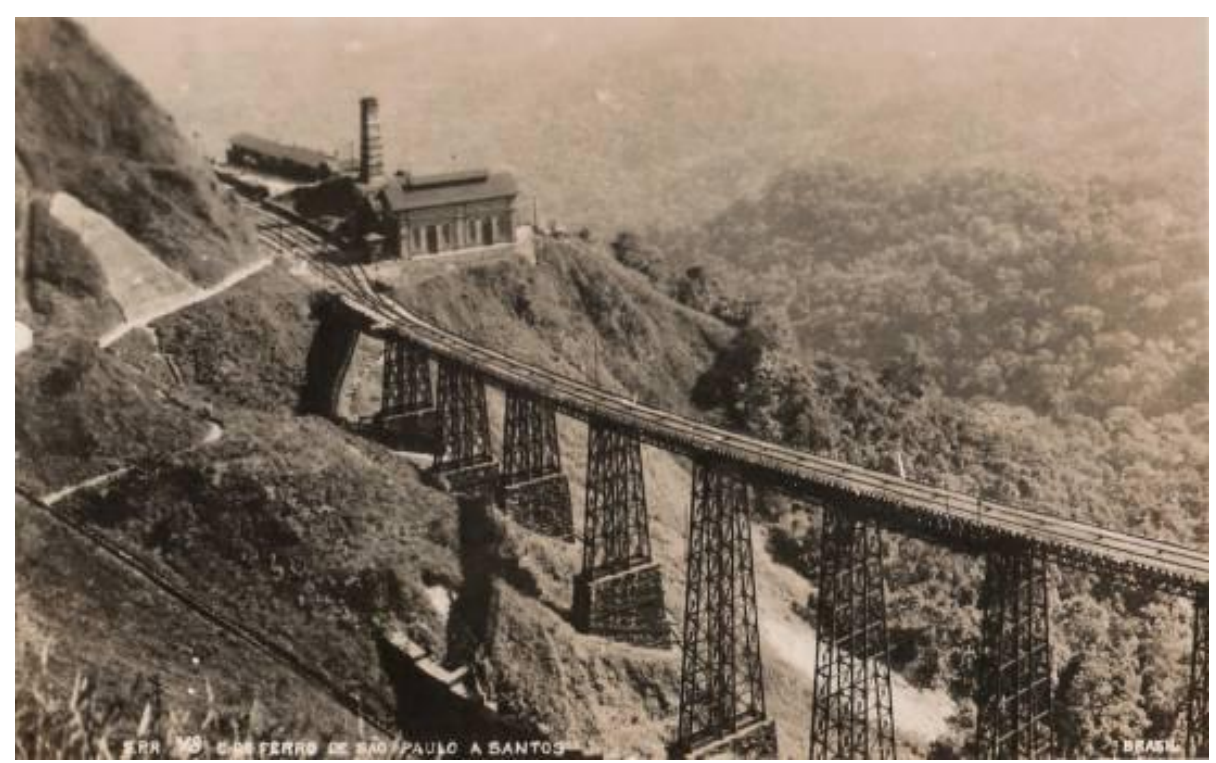

Figura 1: Ponte da São Paulo Railway - Trecho da Serra [29]

Em 30 de janeiro de 1868 foi fundada a Companhia Paulista de Estradas de Ferro, ligando Jundiaí à Campinas, sob a presidência de Clemente Falcão de Sousa Filho; porém sua inauguração aconteceu em 11 de agosto de1882.

No ano de 1872, um grupo de fazendeiros cria uma ferrovia para propiciar o escoamento da produção de café em direção ao interior de São Paulo, nas regiões de Mogi-Mirim e Amparo, ficando conhecida como Companhia Mogiana de Estradas de Ferro.

Com o objetivo de se estabelecer uma ligação ferroviária entre os oceanos Atlântico e Pacífico, através do Estado de Mato Grosso e da Bolívia, em 1904 foi fundada a Estrada de Ferro Noroeste do Brasil. 
Em 30 de setembro de 1957 nasce a RFFSA (Rede Ferroviária Federal Sociedade Anônima), formada pela EFSJ juntamente com a Estrada de Ferro Central do Brasil, responsável por unificar as 42 ferrovias existentes, criando um sistema regional composto por 18 estradas de ferro. Porém, em 1996, a RFFSA foi privatizada e suas linhas foram distribuídas para diversas empresas.

A malha ferroviária chegou a atingir cerca de $38.000 \mathrm{~km}$ por volta de 1960 , porém, a partir de então entrou em declínio e hoje atinge cerca de $30.000 \mathrm{~km}$ em toda sua extensão, sendo mais concentrada na costa brasileira, de Norte a Sul. O transporte de carga por ferrovia corresponde a $26 \%$ de toda a carga transportada. Já é uma realidade no Brasil o aumento de produtividade do modal ferroviário, porém este número passa a ser baixo quando comparado, por exemplo, com os Estados Unidos, que têm $45 \%$ da carga transportada por ferrovia, que é uma matriz extremamente equilibrada e competitiva.

Desde o processo de desestatização das ferrovias, o setor vem em ritmo crescente, apesar de ainda existirem muitos problemas a serem resolvidos, como: conflitos do tráfego ferroviário com veículos rodoviários e pedestres; crescimento desordenado das cidades, impossibilitando manobras dos trens de carga; comprometimento do acesso aos portos pela inexistência de retroáreas capazes de atender à demanda atual e futura; invasões na faixa de domínio, a maior parte ocorrida na época da estatal; e passagens de nível críticas, em média, uma a cada 2,3 quilômetros de ferrovia.

O modal ferroviário tem boas perspectivas de se firmar como meio de transporte bastante competitivo quando se tratar de redução de custos, agilidade e capacidade no transporte de cargas. Porém há necessidade ainda de grandes investimentos para que o País possa ampliar cada vez mais a sua capacidade de transporte ferroviário, a fim de tornar a sua matriz mais eficiente e competitiva.

\subsubsection{Efeito dinâmico em estruturas de pontes}

A preocupação com o problema dinâmico em pontes teve seu início a partir da metade do século XIX, em função do surgimento de veículos mais pesados e velozes. O objetivo dos pesquisadores era propor soluções para o problema de forma simplificada. Em 1849, R. WILLIS deduziu uma equação de movimento com base em um modelo formado por uma massa deslocando-se com velocidade constante sobre 
uma viga simplesmente apoiada, flexível e de massa desprezível. No mesmo ano, STOKES obteve uma solução da equação de movimento por meio de expansão em séries.

INGLIS (1934) estudou o efeito dinâmico em pontes ferroviárias, utilizando o método de análise de harmônicos, que apresenta rápida convergência das séries envolvidas. O autor admitiu que a resposta dinâmica de uma viga bi-apoiada possuía sempre a forma do seu primeiro modo de vibração.

Após a década de 1950, com o surgimento das ferramentas computacionais, e a partir dos anos 70, com o Método dos Elementos Finitos, a análise de vibrações em pontes passou a ser desenvolvida utilizando hipóteses mais sofisticadas.

Em 1979, BLEJWAS et al desenvolveram um procedimento para simular a interação dinâmica veículo-estrutura com o emprego de Multiplicadores de Lagrange, que foram utilizados para satisfazer as restrições cinemáticas; esses multiplicadores não são eliminados, mas determinados como parte integrante da solução numérica. Os autores fazem comparação de soluções analíticas e experimentais, obtendo resultados precisos para todas as análises.

FRÝBA, em 1972, apresentou a solução analítica para problemas de vibração em vigas submetidas a cargas em movimento. $O$ autor também realizou estudos em pontes ferroviárias, analisando sua vida útil, considerando vários parâmetros, como a velocidade do comboio, o amortecimento das vibrações das pontes, a variabilidade no comprimento e na altura da carga em movimento e sua magnitude. Segundo FRÝBA (1980), o aumento da velocidade dos vagões e da distribuição das cargas no eixo longitudinal da ponte, e portanto o aumento do número de ciclos, diminuem a vida útil da estrutura, ou a vida à fadiga; já a consideração do amortecimento mostra um efeito positivo, aumentando a sua vida útil.

Em 1985, OLSSON utilizou o Método dos Elementos Finitos para a resolução de problemas de carga em movimento em pontes. $O$ autor considerou a interação veículo-ponte por meio de elementos finitos, com matrizes dependentes do tempo. A resposta da ponte foi formulada em termos de coordenadas modais, reduzindo assim o número de equações a serem resolvidas dentro de cada intervalo de tempo.

LIN et al (1990) também utilizaram o Método dos Elementos Finitos para a análise de vigas em regime elástico submetidas a cargas dinâmicas induzidas pelo movimento arbitrário de um sistema massa-mola-amortecedor. 
XIA et al (2000) aplicaram o Método dos Elementos Finitos para estudar a interação dinâmica em pontes ferroviárias suspensas. Os autores consideraram um modelo com 27 graus de liberdade para o carro ou vagão, sendo eles os deslocamentos nas direções vertical e horizontal transversal e as rotações em torno dos eixos vertical e horizontais transversal e longitudinal, para a massa suspensa e para cada um dos dois truques, resultando em 15 graus de liberdade e ainda, os deslocamentos horizontal transversal e vertical e rotação em torno do eixo horizontal longitudinal para cada um dos quatro eixos, somando-se mais 12 graus de liberdade. O autor analisou a interação dinâmica entre a ponte e o comboio por meio de forças de contato entre as rodas e os trilhos.

No mesmo ano, ZHANG et al estudaram a interação dinâmica trem-ponte através de modelação numérica, considerando as irregularidades nos trilhos, descritas por funções de densidade espectral geradas pela transformada inversa de Fourier. Segundo os autores, as irregularidades nos planos vertical e horizontal dos trilhos têm importante influência na interação dinâmica trem-ponte.

Em 2003, XIA et al estudaram a resposta dinâmica em pontes pênseis, considerando a passagem dos trens e a influência do vento. De acordo com este trabalho, para velocidades do vento acima de $60 \mathrm{~m} / \mathrm{s}$, as respostas dinâmicas da ponte são dominadas pelas forças do vento, além do que a presença de ventos fortes afeta consideravelmente a segurança da passagem do trem e o conforto dos passageiros; já para velocidades menores do vento $(25 \mathrm{~m} / \mathrm{s})$, a passagem do trem passa a dominar a resposta dinâmica em relação aos seus deslocamentos verticais.

GARINEl et al (2007) analisaram as deformações em pontes de pequeno e médio porte utilizando um modelo de viga simplesmente apoiada submetida a cargas variáveis no tempo. Os autores consideraram a influência do número de vagões, os efeitos da velocidade, da frequência e da fase de harmônicos. A presença de vários componentes harmônicos conduz a fenômenos de amplificação muito mais frequentes do que quando apenas um componente está presente. Os resultados evidenciaram a possibilidade de efeitos de grande amplificação devido às condições de ressonância, que ocorrem em altas velocidades. Concluíram que é necessário reduzir os componentes harmônicos transmitidos pelos eixos dos carros ou vagões através da introdução de amortecedores no sistema de suspensão dos trens, e garantir uma boa manutenção para o sistema trem-trilho. 
Em 2008, CORREA utilizou um modelo com nove graus de liberdade para o vagão a fim de estudar o problema das vibrações induzidas em estruturas de aço em pontes ferroviárias. $O$ autor considerou a influência da interação dinâmica trem-trilhosdormentes-estrutura, as irregularidades geométricas, aleatórias e determinísticas nas rodas e nos trilhos, e a utilização de sistemas alternativos para atenuação das vibrações. Forte influência na resposta dinâmica da estrutura se deve às irregularidades geométricas nos trilhos e nas rodas. Percebe-se que as irregularidades nas rodas são mais prejudiciais à estrutura devido aos impactos causados pelas "mossas" das rodas sobre os trilhos, resultando em grandes picos de deslocamentos e de esforços solicitantes na estrutura.

Em 2009, MAJKA et al realizaram uma pesquisa a fim de avaliar a resposta dinâmica de uma ponte existente submetida à passagem de diferentes comboios e diferentes velocidades, considerando as irregularidades nos trilhos. De acordo com este trabalho, as respostas laterais da ponte foram fortemente afetadas pelas irregularidades, uma vez que estas foram as principais fontes de excitação na direção lateral; os graus de liberdade bounce ou heave (deslocamento vertical) e pitch (rotação em torno do eixo transversal) foram menos sensíveis às irregularidades que os graus sway (deslocamento transversal), yaw (rotação em torno do eixo vertical) e roll (deslocamento em torno do eixo longitudinal). As respostas da ponte cresceram consideravelmente quando a velocidade do trem estava próxima à velocidade crítica, em que ocorreu ressonância com um harmônico de elevada ordem, sendo esta velocidade muito maior do que a máxima operacional. A Figura 2 Apresenta um esquema com os graus de liberdade citados.

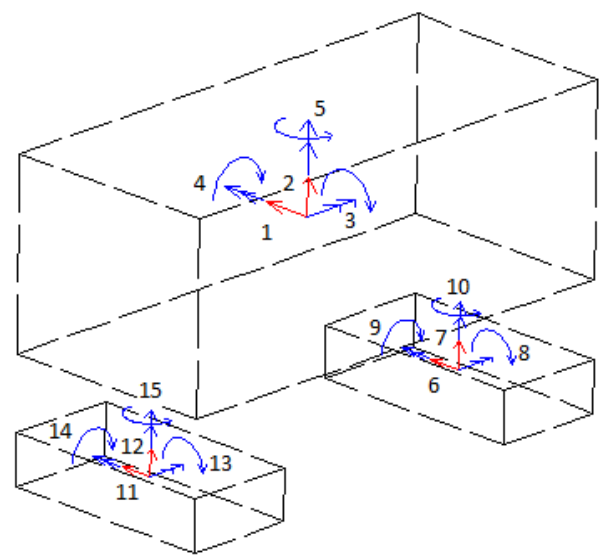

1,6 e 11: Deslocamento horizontal transversal (sway)

2, 7 e 12: Deslocamento vertical (bounce)

3, 8 e 13: Rotação em torno do eixo horizontal longitudinal (roll)

4, 9 e 14: Rotação em torno do eixo horizontal transversal ( $p$ itch)

5, 10 e 11: Rotação em torno do eixo vertical (yaw)

Figura 2: Graus de liberdade considerados neste estudo 
Ainda em 2009, LIU et al também avaliaram os efeitos da interação dinâmica ponte-trem na resposta da ponte durante a passagem do trem, considerando os fatores: relação entre a massa do carro ou vagão e da ponte, relação entre a frequência natural do carro ou vagão e da ponte, a velocidade do trem e a taxa de amortecimento da ponte. Este estudo mostra que a amplificação dinâmica alcança seu valor máximo quando o trem está em sua velocidade crítica, em que a passagem de uma série de eixos regularmente espaçados excita algum modo da ponte.

\subsection{OBJETIVOS E JUSTIFICATIVAS}

O sistema de transporte sobre trilho exerce um papel fundamental no desenvolvimento das cidades e regiões servidas, podendo movimentar cargas ou conduzir pessoas de sua origem a seu destino no menor tempo possível, com o menor consumo energético, sendo meio alternativo ao consumo de petróleo, determinando uma menor emissão de gases nocivos para a atmosfera e ainda, com condições adequadas de segurança e conforto. O transporte sobre trilhos é um modal que apresenta características importantes para se alcançar este desenvolvimento, como a utilização de vias exclusivas que evitem a perda de tempo com engarrafamentos, o emprego de um sistema de monitoramento que permita otimizar o uso das vias, um baixo nível de poluição ambiental e uma grande capacidade de transporte de usuários e mercadorias, se comparado com o modal rodoviário. Todos estes fatores evidenciam o transporte ferroviário como um meio de fácil e rápida movimentação de pessoas e cargas entre cidades, países e continentes, conduzindo ao crescimento econômico e à coesão social.

O uso do modal ferroviário exige a construção de pontes, viadutos e túneis para desvio de construções e obstáculos existentes no traçado da linha férrea e, como os sistemas estruturais empregados estão se tronando cada vez mais esbeltos e, portanto, mais suscetíveis a vibrações, a análise dinâmica é cada vez mais requerida como ferramenta de projeto.

Este trabalho definiu como objetivo específico estudar as vibrações em pontes ferroviárias, produzidas pela passagem da composição de um trem de passageiros, ou TUE (Trem Unidade Elétrico) típico. Para isto, será apresentada uma metodologia simplificada para a análise dinâmica, considerando o veículo e a ponte desacoplados, 
de forma a facilitar a análise de interação de esforços e ainda, podendo utilizar as ferramentas disponíveis no mercado.

Para simulação da carga dinâmica produzida pelos vagões de uma composição, partir-se-á do modelo proposto por CORREA (2008), em que foram considerados os graus de liberdade de deslocamento vertical (bounce) e rotações em torno dos eixos longitudinal (roll) e transversal (pitch), tanto para o vagão, quanto para os dois truques, totalizando nove graus liberdade. Serão acrescentados aqui, os graus de liberdade referentes ao deslocamento transversal horizontal (sway) e a rotações em torno do eixo vertical (yaw) da composição, sendo esses de particular interesse para a excitação de modos de flexão no plano horizontal da ponte, obtendo-se assim um modelo de quinze graus de liberdade.

Será analisada a influência das irregularidades geométricas nos trilhos sobre a interação dinâmica trem-trilhos, nos planos horizontal e vertical, e nas rodas.

Por fim, as respostas dinâmicas obtidas serão comparadas com os valores de referência utilizados na norma brasileira para projeto de pontes ferroviárias (NBR 7187).

\subsection{METODOLOGIA}

Neste trabalho, será apresentada uma metodologia de análise dinâmica simplificada para o estudo das vibrações em pontes ferroviárias produzidas pela passagem da composição de um TUE. Será utilizado um estudo de caso, como aplicação da metodologia apresentada, considerando um modelo aprimorado do vagão de CORREA (2008), com quinze graus de liberdade, a saber, os deslocamentos vertical (bounce) e transversal (sway) e as rotações em torno dos eixos transversal (pitch), longitudinal (roll) e vertical (yaw) da massa suspensa e de cada um dos dois truques.

A interação dinâmica trem-trilhos será estudada com a utilização do software de modelagem estrutural e análise dinâmica ADINA - Automatic Dynamic Incremental Nonlinear Analysis, disponibilizado no Laboratório de Mecânica Computacional da Escola Politécnica da Universidade de São Paulo.

As investigações se iniciarão com a análise modal do sistema mecânicoestrutural do carro, com os quinze graus de liberdade mencionados, bem como de um modelo de alta hierarquia da ponte, usando elementos finitos de casca. Na sequência, 
com a consideração de trilhos e rodas rígidos, porém com imperfeições geométricas, será proposto um modelo de carregamento móvel com cinco graus de liberdade, decorrentes de um processo de condensação estática ao centro de gravidade do carro, sendo dois relativos aos deslocamentos vertical e horizontal transversal e três relativos às rotações com relação aos eixos transversal, longitudinal e vertical do carro, aplicados em um ponto que percorre com uma determinada velocidade um modelo unifilar da ponte, construído com elementos finitos de barra 3D.

As irregularidades geométricas dos trilhos e rodas poderão potencialmente excitar os modos de vibração da ponte. Assim, poderão ser analisados os efeitos dinâmicos nos tabuleiros de pontes ferroviárias, confrontando-os com os decorrentes da utilização dos coeficientes de impacto adotados pela norma brasileira (NBR 7187). 


\section{CARGAS MÓVEIS FERROVIÁRIAS}

\subsection{DESCRIÇÃO DOS TIPOS DE CARROS OU VAGÕES FERROVIÁRIOS UTILIZADOS NO BRASIL}

Os carros ferroviários destinados ao transporte urbano de passageiros, denominados TUE (Trem Unidade Elétrico), são o objeto específico desta dissertação. São compostos por três a quatro vagões, sendo um carro motor, um ou dois carrosreboque e outro carro-reboque com cabine de condução. Uma composição ferroviária normalmente é constituída por dois TUE's (Figura 3). Estes carros são constituídos basicamente por: rodas, eixos, truques, engates, caixa e sistema de controle.

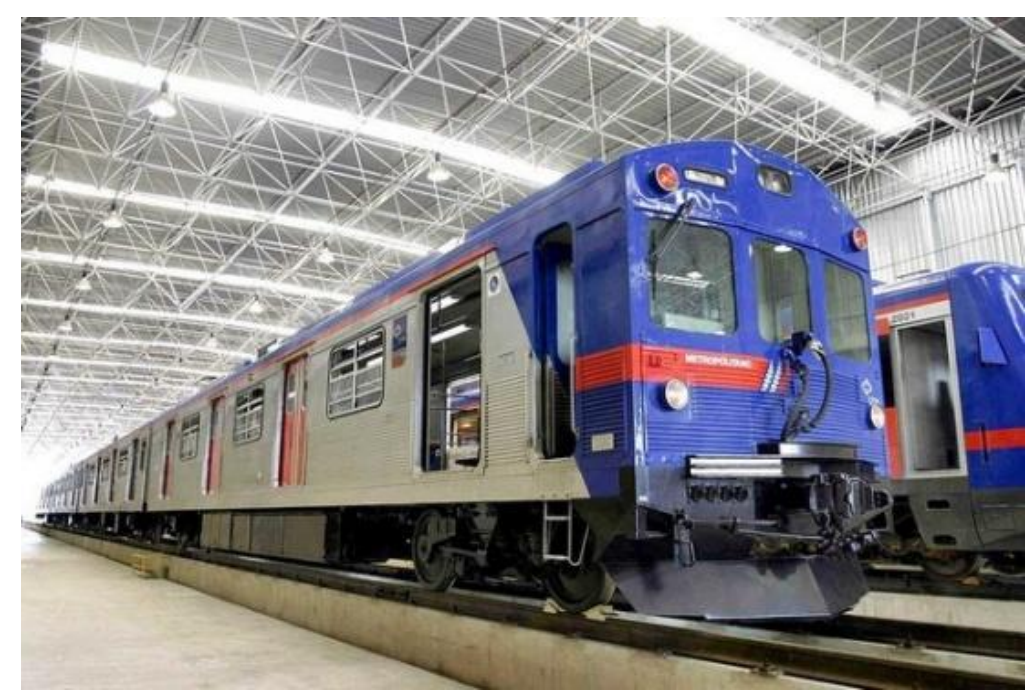

Figura 3: Trem urbano em São Paulo (CPTM) [23]

As rodas são em aço especial e possuem diâmetro de $965 \mathrm{~mm}$. O conjunto de um eixo e duas rodas, uma em cada extremidade, é chamado de rodeiro, que recebe as cargas oriundas da caixa do carro através dos mancais. Dois conjuntos de rodeiros e mais o sistema de suspensão formam os truques, sobre os quais repousa a caixa do carro por meio de pivôs e piões.

O truque do trem é uma estrutura giratória, de base rígida, sobre a qual se apóiam as estruturas (caixas) dos carros, vagões e locomotivas. A suspensão é constituída por molas e guias colocadas entre a armação e os mancais de rolamentos. A armação se apóia em peças com ajuste nas molas e estas, por sua vez, se apóiam na caixa que envolve os rolamentos colocados na ponta de cada eixo. A absorção dos 
impactos laterais na deformação das molas e das guias permite tráfego em velocidade mais uniforme. Com isso, a acomodação do truque é feita com menos desgastes e menor possibilidade de danos.

Os truques são divididos em truque motor e truque reboque. De maneira geral os dois truques são semelhantes, com exceção de os truques motores apresentarem dois motores de tração.

A título de exemplo, a Figura 4 ilustra o desenho do projeto de um truque motor fabricado pela Cobrasma e a Tabela 1 apresenta suas características.

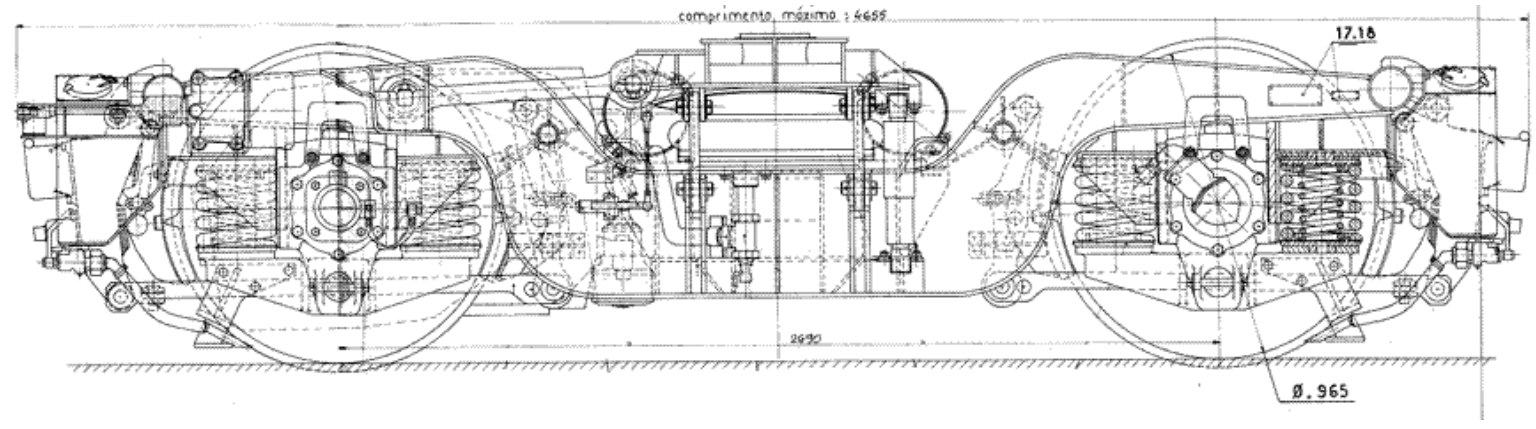

Figura 4: Truque [6]

Tabela 1: Características dos truques [6 e 7]

\begin{tabular}{|l|c|c|}
\hline \multicolumn{1}{|c|}{ Características } & Truque Motor & Truque Reboque \\
\hline Distância entre eixos & $2,69 \mathrm{~m}$ & $2,42 \mathrm{~m}$ \\
\hline Comprimento máximo & $4,655 \mathrm{~m}$ & $4,36 \mathrm{~m}$ \\
\hline Altura máxima & $1,00 \mathrm{~m}$ & $1,00 \mathrm{~m}$ \\
\hline Largura máxima & $2,922 \mathrm{~m}$ & $2,896 \mathrm{~m}$ \\
\hline Diâmetro das rodas & $0,965 \mathrm{~m}$ & $0,965 \mathrm{~m}$ \\
\hline Peso total & $140 \mathrm{kN}$ & $75 \mathrm{kN}$ \\
\hline Rigidez suspensão primária & $6548,78 \mathrm{kN} / \mathrm{m}$ & $5115,2 \mathrm{kN} / \mathrm{m}$ \\
\hline Rigidez suspensão secundária & $1751,31 \mathrm{kN} / \mathrm{m}$ & $1550,38 \mathrm{kN} / \mathrm{m}$ \\
\hline
\end{tabular}

Durante a movimentação dos veículos (locomotivas e vagões), uns transmitem aos outros diferentes esforços de tração e compressão, devido às mudanças de velocidade (aceleração e frenagem). Os engates elásticos são utilizados para acoplar um vagão ao outro, absorvendo esforço de tração ou compressão e evitando choques violentos.

Para efeito de completude, mencionam-se, agora, os trens para o transporte de cargas (vagões), embora não sejam objeto específico desta dissertação. São constituídos por uma locomotiva, sendo no Brasil a mais usual a diesel-elétrica, e uma grande quantidade de vagões. 
Nas locomotivas diesel-elétricas, o motor diesel aciona um gerador que produz a energia elétrica destinada aos motores de tração localizados nos truques e acoplados às rodas motrizes por engrenagens. Especialmente a partir da década de 1970 passou-se a utilizar amplamente no Brasil o alternador, produzindo corrente alternada a ser retificada e enviada aos motores de tração de corrente contínua. Uma tecnologia mais recente é a dos motores de tração à corrente alternada, já comum em diversas ferrovias da América do Norte, mas ainda não utilizada no Brasil.

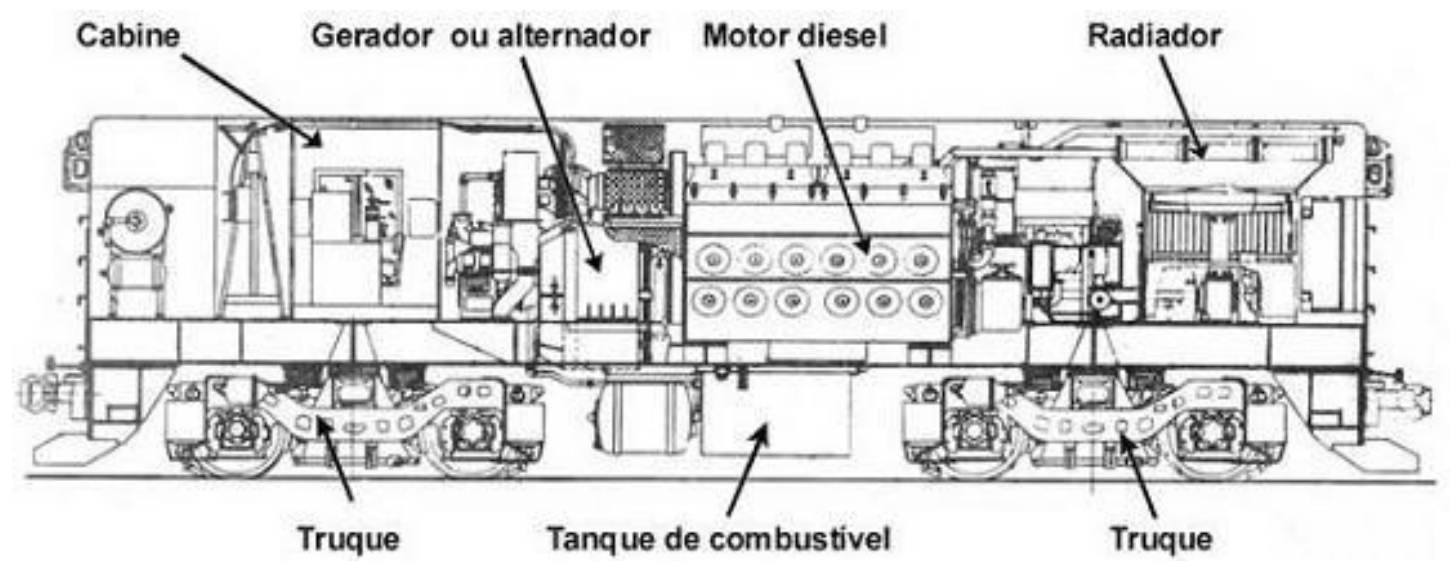

Figura 5: Esquema de uma típica locomotiva diesel-elétrica [22]

As figuras seguintes apresentam alguns dos principais modelos de locomotivas diesel-elétricas em operação em várias ferrovias do Brasil.
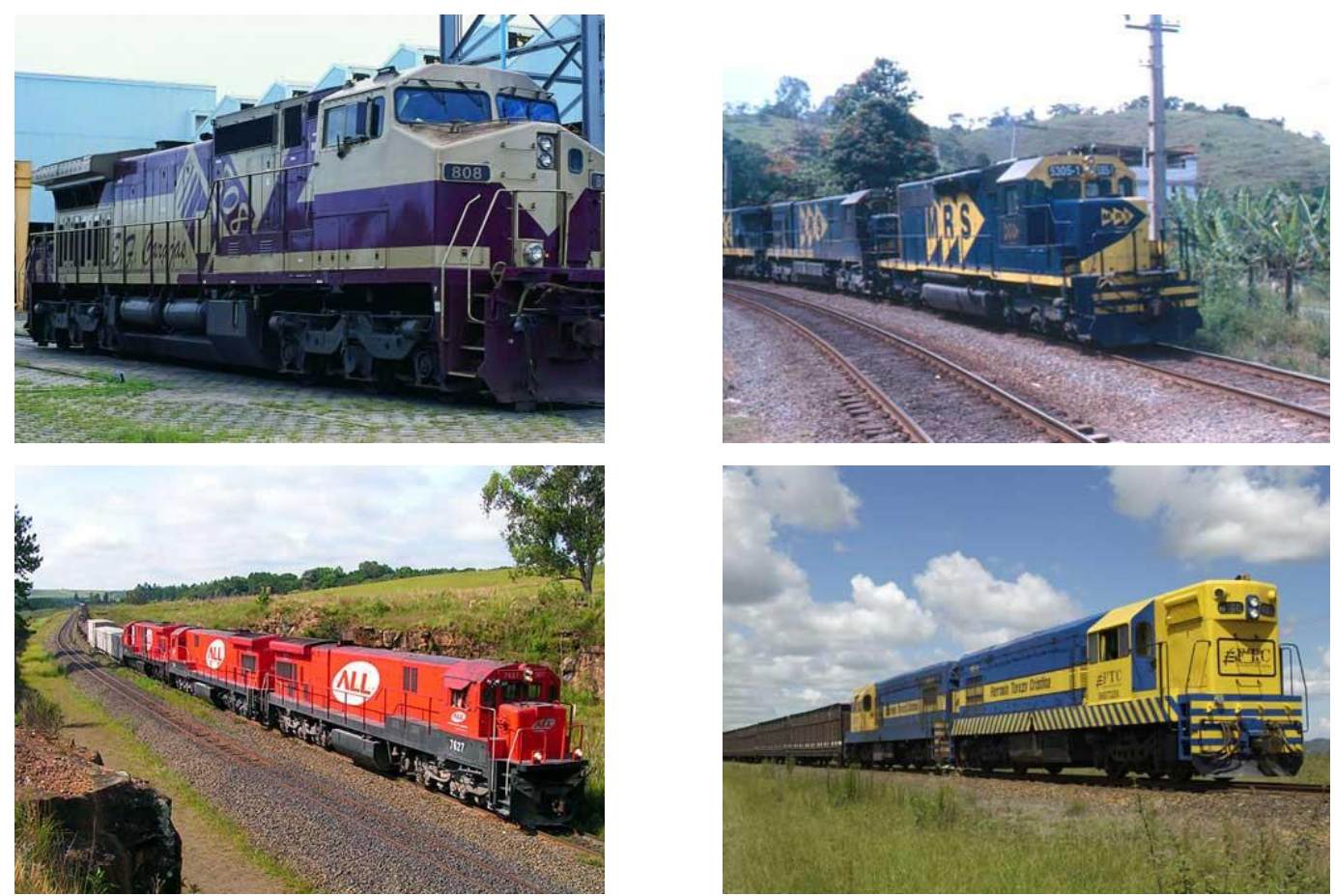

Figura 6: Locomotivas diesel-elétricas utilizadas no Brasil [22] 
O vagão é a parte do material rodante que é rebocada e também responsável pelo transporte da carga. Os vagões são basicamente classificados em função do tipo de produto a ser transportado (grãos, minérios, líquidos, ensacados), tipo de terminal de carga e descarga (equipamentos, pontes, correias), tipo de proteção exigida pelo cliente final, condições geométricas e operacionais da ferrovia ou ferrovias nas quais ele irá circular e interação com os demais vagões existentes nas frotas de seu proprietário. De acordo com a ANTF (Associação Nacional de Transporte Ferroviário) [22], os tipos de vagões mais utilizados no Brasil são:

a) Vagões tipo gôndola - utilizados no transportes de granéis sólidos e produtos diversos que podem ser expostos ao tempo;

b) Vagões fechados - utilizados no transportes de granéis sólidos, ensacados, caixarias, cargas unitizadas e transporte de produtos em geral que não podem ser expostos ao tempo;

c) Vagões tipo hopper - fechados, para o transporte de granéis corrosivos e granéis sólidos que não podem ser expostos ao tempo, e abertos, para o transporte de granéis que podem ser expostos ao tempo;

d) Vagões tipo isotérmico - utilizados no transporte de produtos congelados em geral;

e) Vagões tipo plataforma - utilizados no transporte de conteiners, produtos siderúrgicos, grandes volumes, madeiras, peças de grandes dimensões;

f) Vagões tipo tanque - utilizados no transporte de soja, derivados de petróleo claros e líquidos não corrosivos em geral;

g) Vagões especiais - utilizados no transporte de produtos com características de transporte bem distintas das anteriores. 


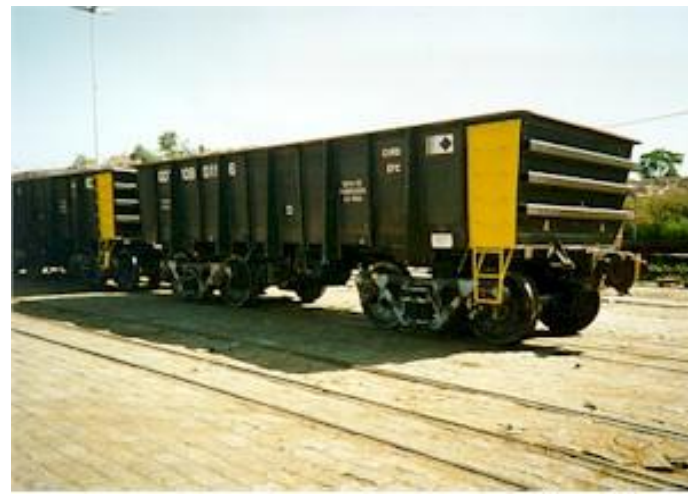

Vagão gôndola [25]

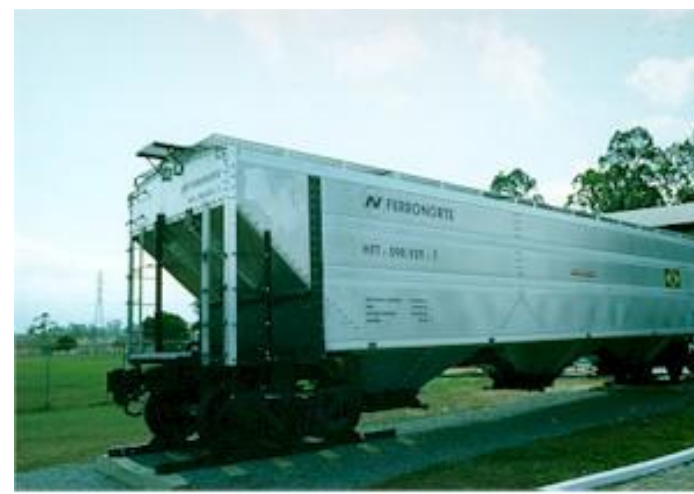

Vagão Hopper [27]

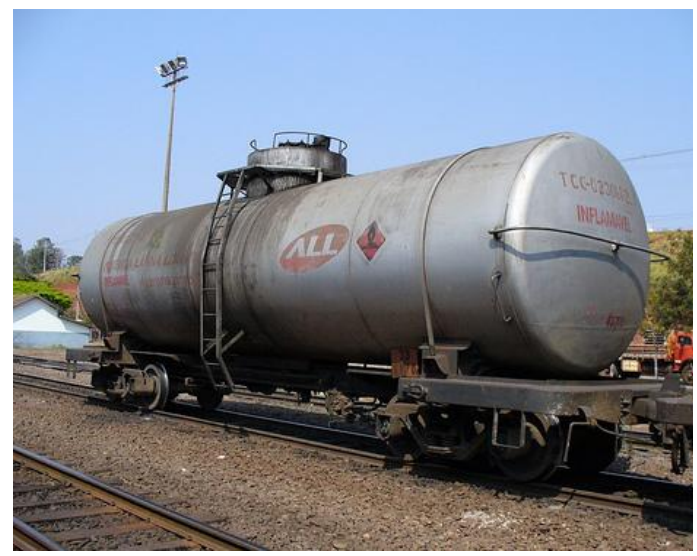

Vagão tanque [26]

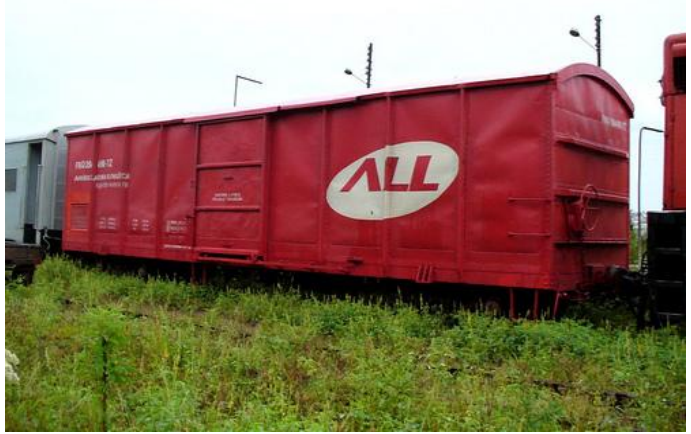

Vagão fechado [26]

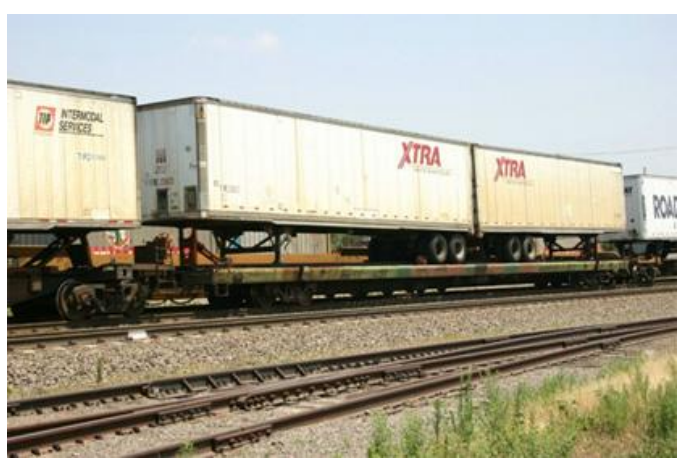

Vagão plataforma [27]

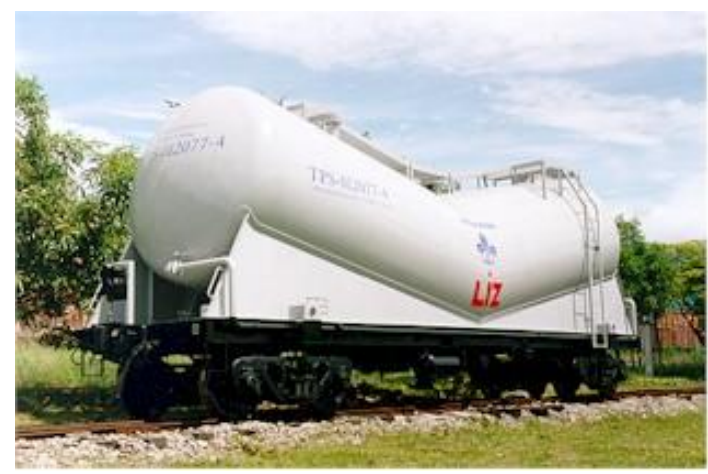

Vagão especial [25]

Figura 7: Tipos de vagões

\subsection{MODELO DE CARGA MÓVEL PRESCRITO NA NBR-7189}

A NBR-7189 Cargas móveis para o projeto estrutural de ferrovias [1] estabelece quatro classes de trens-tipo que são relacionadas a seguir:

a) TB-360: para ferrovias sujeitas a transporte de minério de ferro ou outros carregamentos equivalentes;

b) TB-270: para ferrovias sujeitas a transporte de carga geral; 
c) TB-240: para ser adotado somente na verificação de estabilidade e projeto de reforço de obras existentes;

d) TB-170: para vias sujeitas exclusivamente ao transporte de passageiros em regiões metropolitanas ou suburbanas.

As características geométricas e os valores das cargas estão mostrados na Figura 8 e na Tabela 2, respectivamente

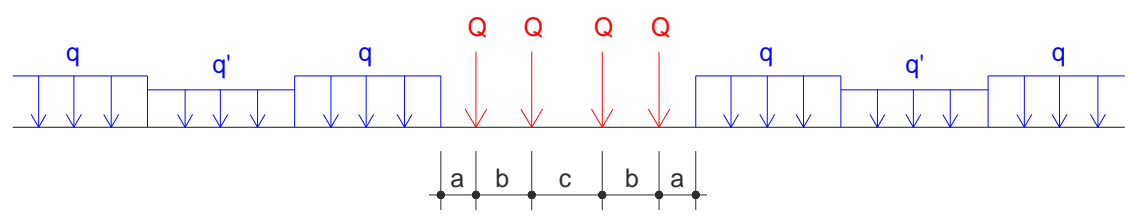

Figura 8: Características geométricas e cargas dos trens-tipo

onde:

$Q=$ peso por eixo;

q e $q^{\prime}=$ forças distribuídas na via, simulando, respectivamente, vagões carregados e descarregados.

Tabela 2: Cargas dos trens-tipo

\begin{tabular}{|c|c|c|c|c|c|c|}
\hline TB & $\mathbf{Q}(\mathbf{k N})$ & $\mathbf{q}(\mathbf{k N} / \mathbf{m})$ & $\mathbf{q}^{\prime}(\mathbf{k N} / \mathbf{m})$ & $\mathbf{a}(\mathbf{m})$ & $\mathbf{b}(\mathbf{m})$ & $\mathbf{c ~ ( m )}$ \\
\hline 360 & 360 & 120 & 20 & 1,00 & 2,00 & 2,00 \\
\hline 270 & 270 & 90 & 15 & 1,00 & 2,00 & 2,00 \\
\hline 240 & 240 & 80 & 15 & 1,00 & 2,00 & 2,00 \\
\hline 170 & 170 & 25 & 15 & 11,00 & 2,50 & 5,00 \\
\hline
\end{tabular}

Segundo a A.R.E.A (American Railway Engineering Association), existe ainda o modelo Cooper 80, utilizado pela Companhia Vale do Rio Doce (CVRD), conforme a Figura 9.

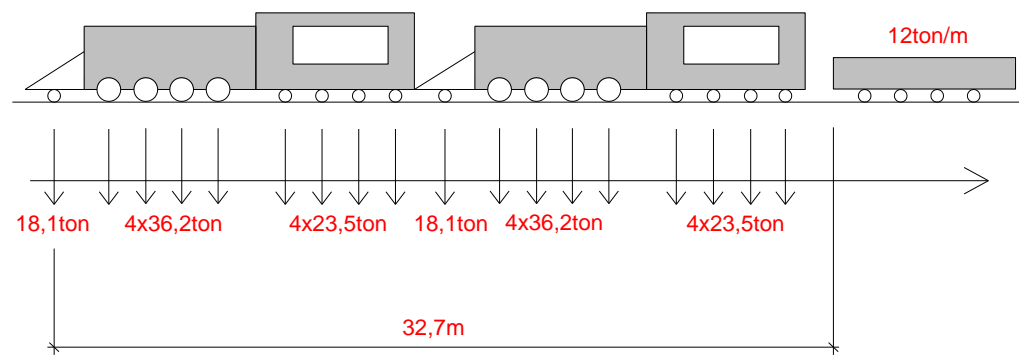

Figura 9: Modelo Cooper 80 [28]

A NBR-7187 Projeto de pontes de concreto armado e de concreto protendido Procedimento [2] considera que o efeito dinâmico de cargas móveis pode ser 
modelado com cargas quase-estáticas, por meio da multiplicação de valores de referência pelo coeficiente de impacto, nos elementos estruturais de obras ferroviárias, definido a seguir:

$$
\varphi=0,001 \times(1600-60 \sqrt{\ell}+2,25 \ell) \geq 1,2
$$

onde:

$\ell=$ comprimento de cada vão teórico do elemento carregado, qualquer que seja o sistema estrutural, em metros.

Note que para

$\ell>150,0 m \quad \varphi \cong 1,2$

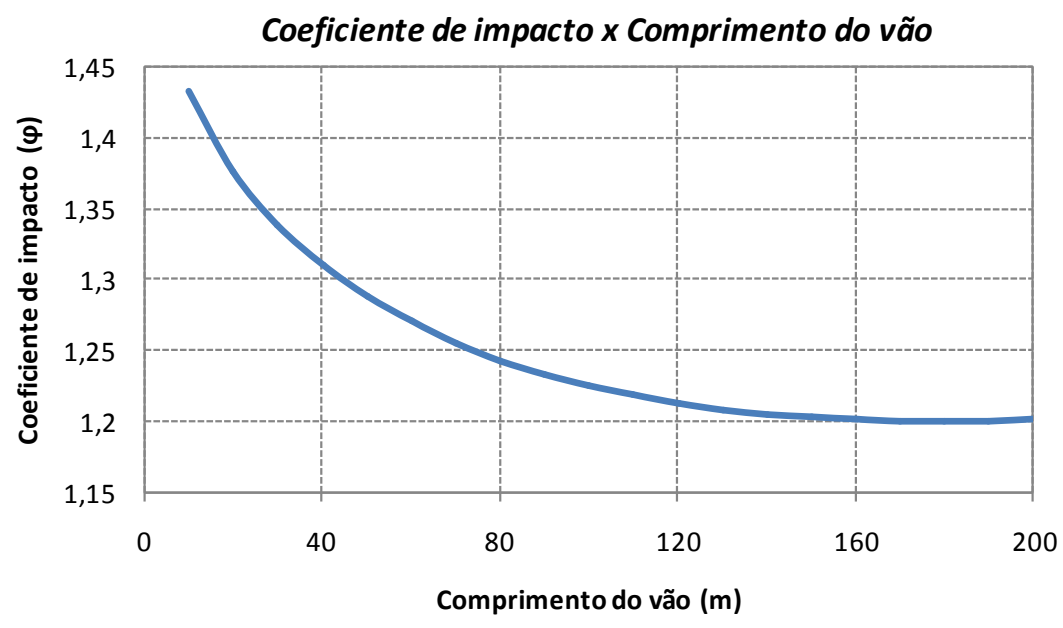

Figura 10: Diagrama do Coeficiente de impacto x Comprimento do vão da ponte

No caso de vãos desiguais, em que o menor vão seja igual ou superior a 70\% do maior, permite-se considerar um vão ideal equivalente à média aritmética dos vãos teóricos. No caso de vigas em balanço, $\ell$ é tomado igual a duas vezes o seu comprimento.

Com relação ao choque lateral provocado pelas rodas dos trens, este é equiparado a uma força horizontal móvel, aplicada na altura do topo dos trilhos, normal ao eixo da linha, com um valor característico igual a $20 \%$ da carga do eixo mais pesado. Em pontes com mais de uma linha, esta ação só é considerada em uma delas.

Cabe comentar ainda que, diferentemente do que a norma brasileira especifica, o Eurocode EN 1991-2 considera um modelo de carregamento específico para cada condição, com amplificadores embutidos nos valores das cargas prescritas. No caso 
de carregamento dinâmico, é utilizado o modelo de carga HSLM, para representar trens de alta velocidade. 


\section{PROCEDIMENTOS DE MODELAGEM}

\subsection{MODELAGEM VEICULAR}

Para a modelagem do carro, partir-se-á do modelo utilizado por CORREA (2008), com nove graus de liberdade, sendo três graus referentes ao deslocamento vertical (bounce), três graus de rotações em torno do eixo transversal (pitch) e três rotações em torno do eixo longitudinal (roll), todos os movimentos analisados para o vagão e os dois truques. Serão acrescentados seis graus de liberdade: três de deslocamento horizontal transversal (sway) e três de rotação em torno do eixo vertical (yaw). A Figura 11 apresenta o modelo dinâmico utilizado; os parâmetros encontramse definidos na Tabela 3.

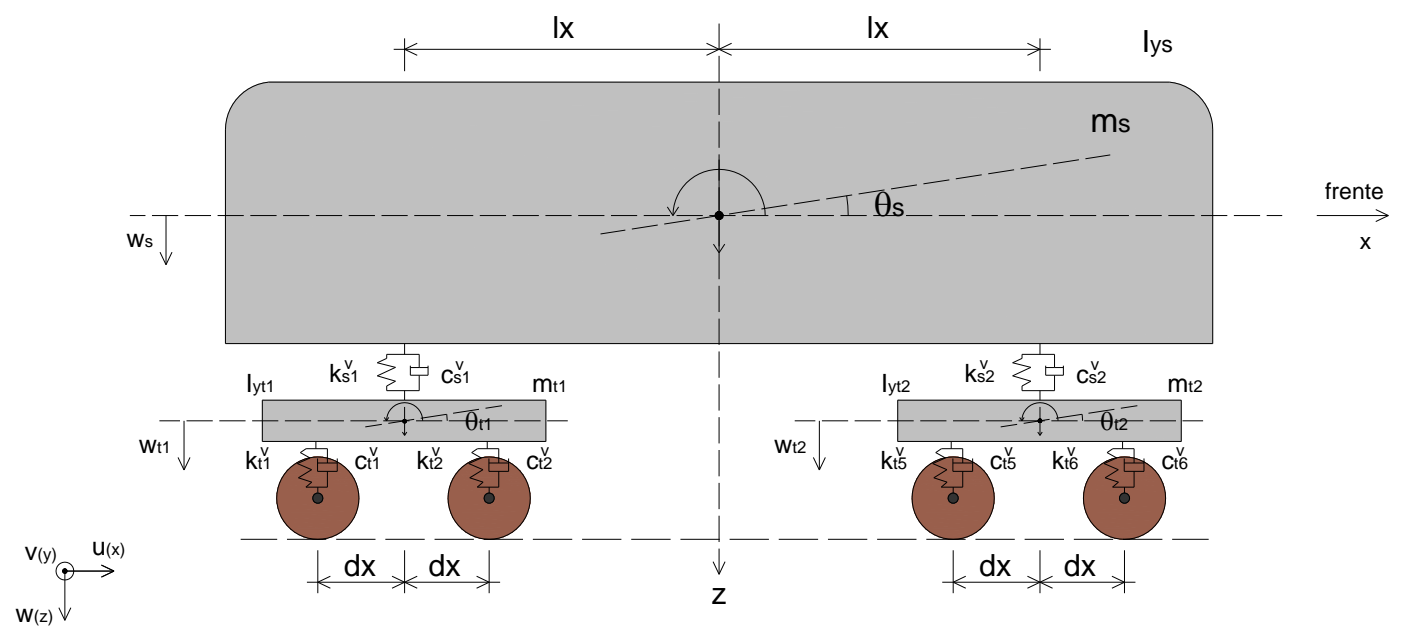

a)

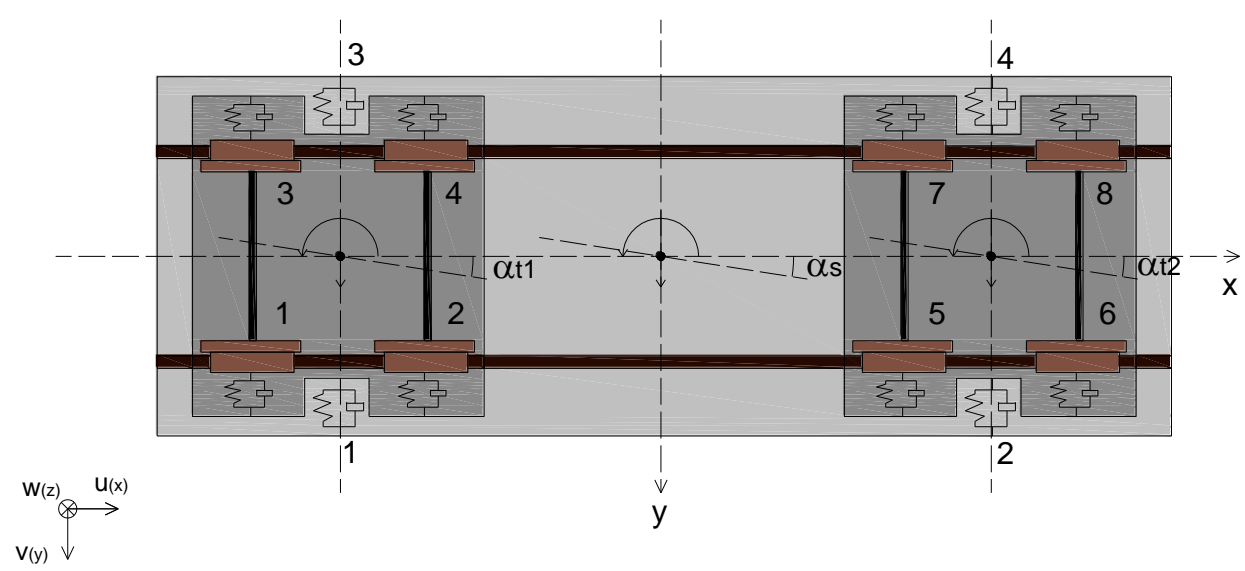

b) 


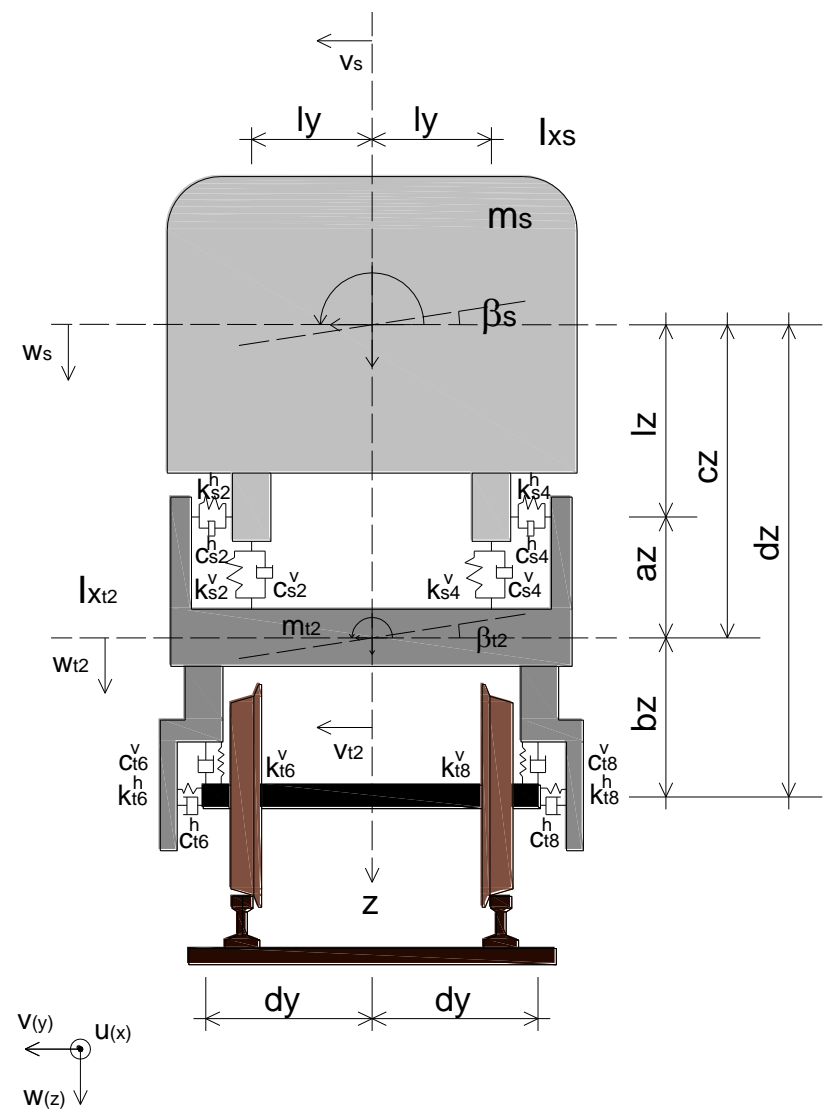

c)

Figura 11: Modelo dinâmico do carro

Em geral, as companhias de trens urbanos utilizam a composição de dois TUE's, sendo cada um deles constituído por dois carros motor e dois carros reboque. Neste trabalho, cujo objetivo é apresentar uma metodologia para análise dinâmica de pontes ferroviárias, serão utilizados dois TUE's compostos por quatro vagões iguais, sem a distinção entre carros motor e reboque.

Neste modelo dinâmico tridimensional, com quinze graus de liberdade para cada carro, são consideradas as massas suspensa e as dos dois truques (dianteiro e traseiro), sendo tratados como corpos rígidos e não se levando em conta a massa das rodas. Foi admitido que as rodas apresentam contato contínuo com os trilhos e que são indeformáveis, sendo deslocáveis devido à presença das irregularidades nas rodas e nos trilhos.

$\mathrm{Na}$ falta de informações geométricas e mecânicas completas para vagões utilizados nas ferrovias brasileiras, será utilizado o modelo veicular apresentado em XIA et al (2000), cujos parâmetros mecânicos e dinâmicos são apresentados na Tabela 3. 
Tabela 3: Parâmetros mecânicos e dinâmicos do carro

\begin{tabular}{|l|c|c|}
\hline \multicolumn{1}{|c|}{ Parâmetro } & Unidade & Valor \\
\hline Massa do vagão $\left(\mathrm{m}_{\mathrm{s}}\right)$ & $\mathrm{kg}$ & 50990 \\
\hline Momento de inércia roll do vagão $\left(\mathrm{I}_{\mathrm{xs}}\right)$ & $\mathrm{t} \cdot \mathrm{m}^{2}$ & 154,83 \\
\hline Momento de inércia pitch do vagão $\left(\mathrm{I}_{\mathrm{ys}}\right)$ & $\mathrm{t} \cdot \mathrm{m}^{2}$ & 1958,7 \\
\hline Momento de inércia yaw do vagão $\left(\mathrm{I}_{z \mathrm{~s}}\right)$ & $\mathrm{t} \cdot \mathrm{m}^{2}$ & 1875,3 \\
\hline Massa do truque $\left(\mathrm{m}_{\mathrm{t}}\right)$ & $\mathrm{kg}$ & 4360 \\
\hline Momento de inércia roll do truque $\left(\mathrm{I}_{\mathrm{xt}}\right)$ & $\mathrm{t} \cdot \mathrm{m}^{2}$ & 1,47 \\
\hline Momento de inércia pitch do truque $\left(\mathrm{I}_{\mathrm{yt}}\right)$ & $\mathrm{t} \cdot \mathrm{m}^{2}$ & 3,43 \\
\hline Momento de inércia yaw do truque $\left(\mathrm{I}_{z \mathrm{zt}}\right)$ & $\mathrm{t} \cdot \mathrm{m}^{2}$ & 5,07 \\
\hline Rigidez vertical da suspensão primária $\left(\mathrm{k}_{\mathrm{t}}{ }^{\mathrm{v}}\right)$ & $\mathrm{kN} / \mathrm{m}$ & 2976 \\
\hline Rigidez lateral da suspensão primária $\left(\mathrm{k}_{\mathrm{t}}{ }^{\mathrm{h}}\right)$ & $\mathrm{kN} / \mathrm{m}$ & 20000 \\
\hline Rigidez vertical da suspensão secundária $\left(\mathrm{k}_{\mathrm{s}}{ }^{\mathrm{v}}\right)$ & $\mathrm{kN} / \mathrm{m}$ & 1060 \\
\hline Rigidez lateral da suspensão secundária $\left(\mathrm{k}_{\mathrm{s}}{ }^{\mathrm{h}}\right)$ & $\mathrm{kN} / \mathrm{m}$ & 460 \\
\hline Amortecedor vertical da suspensão primária $\left(\mathrm{c}_{\mathrm{t}}^{\mathrm{v}}\right)$ & $\mathrm{kNs} / \mathrm{m}$ & 15 \\
\hline Amortecedor lateral da suspensão primária $\left(\mathrm{c}_{\mathrm{t}}{ }^{\mathrm{h}}\right)$ & $\mathrm{kNs} / \mathrm{m}$ & 15 \\
\hline Amortecedor vertical da suspensão secundária $\left(\mathrm{c}_{\mathrm{s}}{ }^{\mathrm{v}}\right)$ & $\mathrm{kNs} / \mathrm{m}$ & 30 \\
\hline Amortecedor lateral da suspensão secundária $\left(\mathrm{c}_{\mathrm{s}}{ }^{\mathrm{h}}\right)$ & $\mathrm{kNs} / \mathrm{m}$ & 30 \\
\hline Comprimento total do veículo & $\mathrm{m}$ & 22,5 \\
\hline Distância entre dois truques $\left(2 \mathrm{I}_{\mathrm{x}}\right)$ & $\mathrm{m}$ & 15,6 \\
\hline Distância entre dois eixos $\left(2 \mathrm{~d}_{\mathrm{x}}\right)$ & $\mathrm{m}$ & 2,5 \\
\hline Distância $\mathrm{I}_{z}$ & $\mathrm{~m}$ & 0,98 \\
\hline Distância $a_{z}$ & $\mathrm{~m}$ & 0,36 \\
\hline Distância $\mathrm{b}_{z}$ & 0,07 \\
\hline Distância $\mathrm{d}_{\mathrm{y}}$ & 0,98 \\
\hline Distância $\mathrm{I}_{\mathrm{y}}$ & $\mathrm{m}$ & 1,12 \\
\hline
\end{tabular}

\subsection{IRREGULARIDADES GEOMÉTRICAS NOS TRILHOS E NAS RODAS}

As vibrações de pontes ferroviárias podem ser afetadas pela presença das irregularidades, que são desvios na faixa de contorno da geometria ideal. As irregularidades resultam em um aumento da carga dinâmica a ser transferida do carro para a ponte. Segundo MAJKA et al (2009), as irregularidades ao longo da via podem ter distribuição quase-periódica ou serem isoladas e distribuídas aleatoriamente. As irregularidades periódicas (Figura 12), como ondulações na superfície do trilho, podem causar vibrações significantes no carro, principalmente quando seu comprimento de 
onde $\lambda$ percorrido a uma velocidade $V$ levar a uma frequência $\frac{2 \pi V}{\lambda}$ coincidente com alguma frequência natural do vagão.

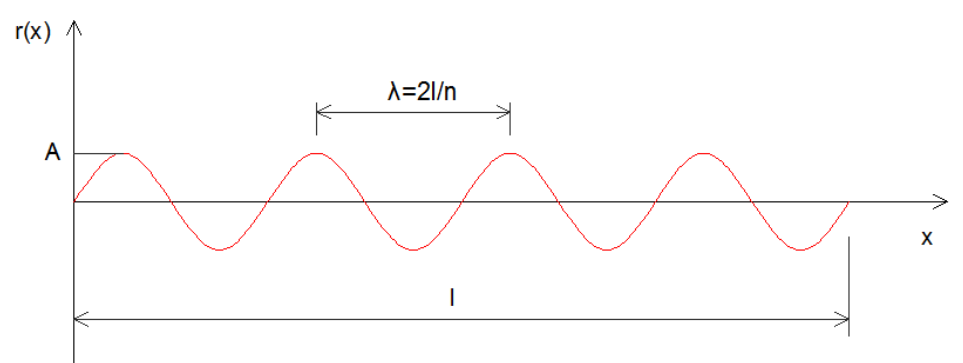

Figura 12: Irregularidade senoidal

Neste trabalho, foram consideradas as irregularidades periódicas nos trilhos, nos planos vertical e horizontal, e nas rodas, sendo defasadas para cada trilho e roda. As irregularidades no plano vertical dos trilhos são causadas pela ação das rodas e ação da corrosão causada pelas intempéries, além da deformação plástica da superfície de rodagem dos trilhos. Já no plano horizontal, as irregularidades são causadas devido à dificuldade de manter o alinhamento dos trilhos durante sua fase de construção e também, devido à flambagem lateral de um trecho da via ou de desvios de traçado no assentamento dos trilhos. Segundo MAJKA et al (2009), as respostas laterais da ponte são fortemente influenciadas pelas irregularidades no plano horizontal, já que estas são as principais fontes de excitação nessa direção, em trechos retilíneos, quando não há esforços centrífugos. No caso das rodas, podem existir achatamentos localizados, ou mossas, na superfície de contato dos aros das rodas devido ao material que as constitui ser menos duro quando comparado ao dos trilhos. Para caracterizar essas imperfeições, são considerados os modelos matemáticos a seguir, conforme apresentado em CORREA (2008).

a) Irregularidade Longitudinal:

Para as irregularidades longitudinais nos planos vertical e horizontal, será considerada a função:

$r(x)=\operatorname{Asen}\left(\frac{2 \pi x}{\lambda}+\phi\right)$

onde:

$A$ : amplitude da irregularidade em $\mathrm{m}$;

$x=V t$ : espaço percorrido pelo carro em $m$;

$V$ : velocidade do carro em $\mathrm{m} / \mathrm{s}$; 
$\lambda=\frac{2 \ell}{n}:$ comprimento de onda;

$\ell$ : comprimento com irregularidades;

$n$ : número de meias ondas em $\ell$ (ver Figura 13);

$\phi$ : ângulo de fase.

Considerando que a frequência da irregularidade depende de $n$ e da velocidade $V$ do trem, conforme indica a expressão:

$\omega_{n}=\frac{2 \pi V}{\lambda}=\frac{n \pi V}{\ell}$

tem-se para o caso de $n$ meias-ondas:

$r(x)=A_{n} \operatorname{sen}\left(\omega_{n} t+\phi\right)$

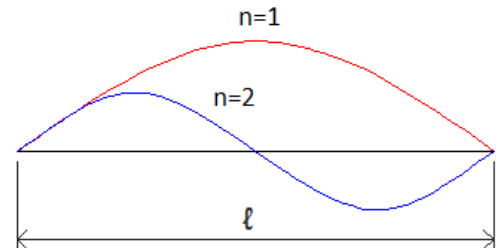

Figura 13: Número de meias ondas no comprimento $\ell$

b) Irregularidade nas rodas

As irregularidades nas rodas, ou mossas, são descritas pela função:

$r(x)$

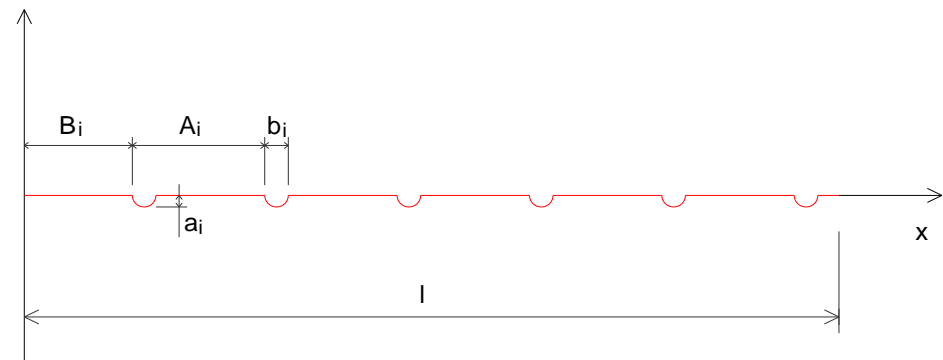

Figura 14: Irregularidades nas rodas

$r(x)=\left\{\begin{array}{cl}\frac{1}{2} a_{i}\left[1-\cos \frac{2 \Pi}{b_{i}}\left(x-k A_{i}-B_{i}\right)\right], & \text { se } \quad B_{i}+k A_{i} \leq x \leq B_{i}+k A_{i}+b_{i} \\ 0, & \text { se } \quad B_{i}+k A_{i}+b_{i}<x<B_{i}+(k+1) A\end{array}\right.$

onde:

$A_{i}, B_{i}, a_{i}$ e $b_{i}$ : indicados na Figura 14;

$i$ : a i-ésima roda com achatamento; 
$k: 0,1,2 \ldots$

Adota-se a seguinte notação (Figura 15):

$A$ : comprimento da circunferência;

$B$ : distância do primeiro impacto da roda em relação ao início da ponte;

$a$ : profundidade do achatamento;

$b$ : comprimento do achatamento.

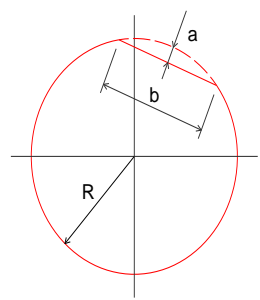

Figura 15: Detalhe da roda com achatamento

\subsection{MODELAGEM ESTRUTURAL}

A ponte utilizada no modelo foi extraída de STUCCHI et al (1989). Trata-se de uma ponte metroviária em concreto armado, com pista dupla, cuja excentricidade da via é de $2,4 \mathrm{~m}$, e com um vão bi-apoiado de $36 \mathrm{~m}$ de comprimento. A seção transversal é em caixão, cujas dimensões e propriedades são apresentadas na Figura 16 e na Tabela 4.

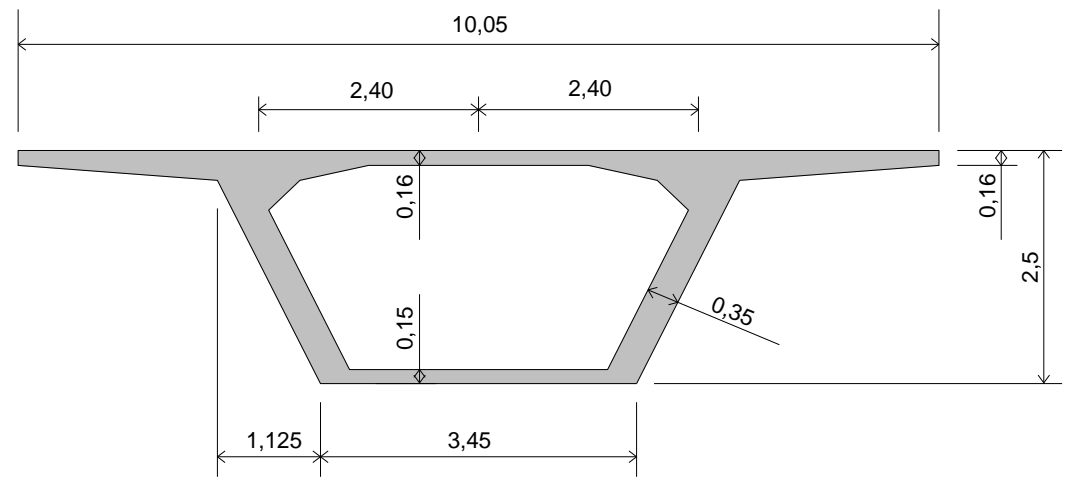

Figura 16: Seção transversal da ponte 
Tabela 4: Propriedades da ponte

\begin{tabular}{|c|c|c|}
\hline$A$ & 4,56 & $\mathrm{~m}^{2}$ \\
\hline $\mathrm{Ix}$ & 3,52 & $\mathrm{~m}^{4}$ \\
\hline $\mathrm{Iy}$ & 28,88 & $\mathrm{~m}^{4}$ \\
\hline $\mathrm{It}$ & 6,68 & $\mathrm{~m}^{4}$ \\
\hline $\mathrm{E}$ & $2,38.10^{10}$ & $\mathrm{~N} / \mathrm{m}^{2}$ \\
\hline $\mathrm{m}$ & $11.169,84$ & $\mathrm{~kg} / \mathrm{m}$ \\
\hline $\mathrm{Ir}_{\mathrm{x}}$ & $39.273,16$ & $\mathrm{~kg} / \mathrm{m}^{2}$ \\
\hline $\mathrm{Ir}_{\mathrm{y}}$ & $1.134 .090,97$ & $\mathrm{~kg} / \mathrm{m}^{2}$ \\
\hline
\end{tabular}

onde:

$A$ : área da seção transversal;

$I x$ : momento de inércia com relação ao eixo horizontal transversal;

$I y:$ momento de inércia com relação ao eixo vertical;

It : momento de inércia à torção;

$E$ : módulo de elasticidade;

$m$ : massa da ponte por unidade de comprimento.

$I r_{x}$ : inércia rotacional em relação ao eixo horizontal transversal;

$I r_{y}$ : inércia rotacional em relação ao eixo vertical. 


\section{FORÇAS DE INTERAÇÃO E EQUAÇÕES DE MOVIMENTO}

\subsection{ASPECTOS GERAIS}

Para a obtenção das forças de interação, realiza-se o estudo do modelo veicular adotando, simplificadamente, tabuleiro rígido indeslocável e contato contínuo entre roda e trilho. A hipótese de tabuleiro rígido pode ser corrigida por um procedimento iterativo, conforme será oportunamente comentado nesta dissertação.

O comboio utilizado é composto por dois conjuntos de quatro carros trafegando a uma velocidade de $20 \mathrm{~m} / \mathrm{s}$, equivalente a $72 \mathrm{~km} / \mathrm{h}$. Evidentemente, trata-se de apenas um estudo de caso para efeito de ilustrar a metodologia de análise.

As irregularidades periódicas para cada um dos trilhos foram consideradas defasadas em $90^{\circ}$, conforme expressões apresentadas no item 3.2. Para as rodas, a variável utilizada para que cada achatamento não ocorresse ao mesmo tempo em todas as rodas foi $B$, conforme já definido em 3.2.

Os esforços obtidos para as reações das rodas são condensados estaticamente no centro de gravidade do carro segundo cinco esforços principais, sendo eles: momentos em torno dos eixos $x, y$ e $z$ e forças nas direções y e $z$. Esses esforços serão aplicados em um modelo estrutural unifilar de baixa hierarquia para a ponte.

\subsection{FORÇAS DE INTERAÇÃO TREM-TRILHO}

As equações de movimento que descrevem o comportamento do trem seguem os parâmetros apresentados na Figura 11. As irregularidades nos trilhos e nas rodas entraram como excitações de suporte aplicadas no contato roda-trilho.

A seguir, são apresentadas as equações de movimento para os quinze graus de liberdade estudados.

a) Para o grau de liberdade de deslocamento vertical (bounce) da massa $\mathrm{m}_{\mathrm{s}}$ :

$$
\begin{aligned}
& m_{s} \ddot{w}_{s}+c_{s 1}^{v}\left(\dot{w}_{s}+\ell_{x} \dot{\theta}_{s}+\ell_{y} \dot{\beta}_{s}-\dot{w}_{t 1}-\ell_{y} \dot{\beta}_{t 1}\right)+c_{s 2}^{v}\left(\dot{w}_{s}-\ell_{x} \dot{\theta}_{s}+\ell_{y} \dot{\beta}_{s}-\dot{w}_{t 2}-\ell_{y} \dot{\beta}_{t 2}\right)+ \\
& c_{s 3}^{v}\left(\dot{w}_{s}+\ell_{x} \dot{\theta}_{s}-\ell_{y} \dot{\beta}_{s}-\dot{w}_{t 1}+\ell_{y} \dot{\beta}_{t 1}\right)+c_{s 4}^{v}\left(\dot{w}_{s}-\ell_{x} \dot{\theta}_{s}-\ell_{y} \dot{\beta}_{s}-\dot{w}_{t 2}+\ell_{y} \dot{\beta}_{t 2}\right)+ \\
& k_{s 1}^{v}\left(w_{s}+\ell_{x} \theta_{s}+\ell_{y} \beta_{s}-w_{t 1}-\ell_{y} \beta_{t 1}\right)+k_{s 2}^{v}\left(w_{s}-\ell_{x} \theta_{s}+\ell_{y} \beta_{s}-w_{t 2}-\ell_{y} \beta_{t 2}\right)+ \\
& k_{s 3}^{v}\left(w_{s}+\ell_{x} \theta_{s}-\ell_{y} \beta_{s}-w_{t 1}+\ell_{y} \beta_{t 1}\right)+k_{s 4}^{v}\left(w_{s}-\ell_{x} \theta_{s}-\ell_{y} \beta_{s}-w_{t 2}+\ell_{y} \beta_{t 2}\right)=F_{w_{s}}
\end{aligned}
$$


b) Para o grau de liberdade de deslocamento transversal (sway) da massa $\mathrm{m}_{\mathrm{s}}$ :

$$
\begin{aligned}
& m_{s} \ddot{v}_{s}+c_{s 1}^{h}\left(\dot{v}_{s}-\ell_{z} \dot{\beta}_{s}-\ell_{x} \dot{\alpha}_{s}-\dot{v}_{t 1}-a_{z} \dot{\beta}_{t 1}\right)+c_{s 2}^{h}\left(\dot{v}_{s}-\ell_{z} \dot{\beta}_{s}+\ell_{x} \dot{\alpha}_{s}-\dot{v}_{t 2}-a_{z} \dot{\beta}_{t 2}\right)+ \\
& c_{s 3}^{h}\left(\dot{v}_{s}-\ell_{z} \dot{\beta}_{s}-\ell_{x} \dot{\alpha}_{s}-\dot{v}_{t 1}-a_{z} \dot{\beta}_{t 1}\right)+c_{s 4}^{h}\left(\dot{v}_{s}-\ell_{z} \dot{\beta}_{s}+\ell_{x} \dot{\alpha}_{s}-\dot{v}_{t 2}-a_{z} \dot{\beta}_{t 2}\right)+ \\
& k_{s 1}^{h}\left(v_{s}-\ell_{z} \beta_{s}-\ell_{x} \alpha_{s}-v_{t 1}-a_{z} \beta_{t 1}\right)+k_{s 2}^{h}\left(v_{s}-\ell_{z} \beta_{s}+\ell_{x} \alpha_{s}-v_{t 2}-a_{z} \beta_{t 2}\right)+ \\
& k_{s 3}^{h}\left(v_{s}-\ell_{z} \beta_{s}-\ell_{x} \alpha_{s}-v_{t 1}-a_{z} \beta_{t 1}\right)+k_{s 4}^{h}\left(v_{s}-\ell_{z} \beta_{s}+\ell_{x} \alpha_{s}-v_{t 2}-a_{z} \beta_{t 2}\right)=F_{v_{s}}
\end{aligned}
$$

c) Para o grau de liberdade de rotação em torno do eixo transversal (pitch) da massa $\mathrm{m}_{\mathrm{s}}$ :

$$
\begin{aligned}
& I_{y s} \ddot{\theta}_{s}+c_{s 1}^{v}\left(\dot{w}_{s}+\ell_{x} \dot{\theta}_{s}+\ell_{y} \dot{\beta}_{s}-\dot{w}_{t 1}-\ell_{y} \dot{\beta}_{t 1}\right) \ell_{x}-c_{s 2}^{v}\left(\dot{w}_{s}-\ell_{x} \dot{\theta}_{s}+\ell_{y} \dot{\beta}_{s}-\dot{w}_{t 2}-\ell_{y} \dot{\beta}_{t 2}\right) \ell_{x}+ \\
& c_{s 3}^{v}\left(\dot{w}_{s}+\ell_{x} \dot{\theta}_{s}-\ell_{y} \dot{\beta}_{s}-\dot{w}_{t 1}+\ell_{y} \dot{\beta}_{t 1}\right) \ell_{x}-c_{s 4}^{v}\left(\dot{w}_{s}-\ell_{x} \dot{\theta}_{s}-\ell_{y} \dot{\beta}_{s}-\dot{w}_{t 2}+\ell_{y} \dot{\beta}_{t 2}\right) \ell_{x}+ \\
& k_{s 1}^{v}\left(w_{s}+\ell_{x} \theta_{s}+\ell_{y} \beta_{s}-w_{t 1}-\ell_{y} \beta_{t 1}\right) \ell_{x}-k_{s 2}^{v}\left(w_{s}-\ell_{x} \theta_{s}+\ell_{y} \beta_{s}-w_{t 2}-\ell_{y} \beta_{t 2}\right) \ell_{x}+ \\
& k_{s 3}^{v}\left(w_{s}+\ell_{x} \theta_{s}-\ell_{y} \beta_{s}-w_{t 1}+\ell_{y} \beta_{t 1}\right) \ell_{x}-k_{s 4}^{v}\left(w_{s}-\ell_{x} \theta_{s}-\ell_{y} \beta_{s}-w_{t 2}+\ell_{y} \beta_{t 2}\right) \ell_{x}=F_{\theta_{s}}
\end{aligned}
$$

d) Para o grau de liberdade de rotação em torno do eixo longitudinal (roll) da massa $\mathrm{m}_{\mathrm{s}}$ :

$$
\begin{aligned}
& I_{x s} \ddot{\beta}_{s}+c_{s 1}^{v}\left(\dot{w}_{s}+\ell_{x} \dot{\theta}_{s}+\ell_{y} \dot{\beta}_{s}-\dot{w}_{t 1}-\ell_{y} \dot{\beta}_{t 1}\right) \ell_{y}+c_{s 2}^{v}\left(\dot{w}_{s}-\ell_{x} \dot{\theta}_{s}+\ell_{y} \dot{\beta}_{s}-\dot{w}_{t 2}-\ell_{y} \dot{\beta}_{t 2}\right) \ell_{y}- \\
& c_{s 3}^{v}\left(\dot{w}_{s}+\ell_{x} \dot{\theta}_{s}-\ell_{y} \dot{\beta}_{s}-\dot{w}_{t 1}+\ell_{y} \dot{\beta}_{t 1}\right) \ell_{y}-c_{s 4}^{v}\left(\dot{w}_{s}-\ell_{x} \dot{\theta}_{s}-\ell_{y} \dot{\beta}_{s}-\dot{w}_{t 2}+\ell_{y} \dot{\beta}_{t 2}\right) \ell_{y}+ \\
& k_{s 1}^{v}\left(w_{s}+\ell_{x} \theta_{s}+\ell_{y} \beta_{s}-w_{t 1}-\ell_{y} \beta_{t 1}\right) \ell_{y}+k_{s 2}^{v}\left(w_{s}-\ell_{x} \theta_{s}+\ell_{y} \beta_{s}-w_{t 2}-\ell_{y} \beta_{t 2}\right) \ell_{y}- \\
& k_{s 3}^{v}\left(w_{s}+\ell_{x} \theta_{s}-\ell_{y} \beta_{s}-w_{t 1}+\ell_{y} \beta_{t 1}\right) \ell_{y}-k_{s 4}^{v}\left(w_{s}-\ell_{x} \theta_{s}-\ell_{y} \beta_{s}-w_{t 2}+\ell_{y} \beta_{t 2}\right) \ell_{y}- \\
& c_{s 1}^{h}\left[\left(\dot{v}_{s}-\ell_{z} \dot{\beta}_{s}-\ell_{x} \dot{\alpha}_{s}\right) \ell_{z}+\left(-\dot{v}_{t 1}-a_{z} \dot{\beta}_{t 1}\right) \ell_{z}\right]-c_{s 2}^{h}\left[\left(\dot{v}_{s}-\ell_{z} \dot{\beta}_{s}+\ell_{x} \dot{\alpha}_{s}\right) \ell_{z}+\left(-\dot{v}_{t 2}-a_{z} \dot{\beta}_{t 2}\right) \ell_{z}\right]- \\
& c_{s 3}^{h}\left[\left(\dot{v}_{s}-\ell_{z} \dot{\beta}_{s}-\ell_{x} \dot{\alpha}_{s}\right) \ell_{z}+\left(-\dot{v}_{t 1}-a_{z} \dot{\beta}_{t 1}\right) \ell_{z}\right]-c_{s 4}^{h}\left[\left(\dot{v}_{s}-\ell_{z} \dot{\beta}_{s}+\ell_{x} \dot{\alpha}_{s}\right) \ell_{z}+\left(-\dot{v}_{t 2}-a_{z} \dot{\beta}_{t 2}\right) \ell_{z}\right]- \\
& k_{s 1}^{h}\left[\left(v_{s}-\ell_{z} \beta_{s}-\ell_{x} \alpha_{s}\right) \ell_{z}+\left(-v_{t 1}-a_{z} \beta_{t 1}\right) \ell_{z}\right]-k_{s 2}^{h}\left[\left(v_{s}-\ell_{z} \beta_{s}+\ell_{x} \alpha_{s}\right) \ell_{z}+\left(-v_{t 2}-a_{z} \beta_{t 2}\right) \ell_{z}\right]- \\
& k_{s 3}^{h}\left[\left(v_{s}-\ell_{z} \beta_{s}-\ell_{x} \alpha_{s}\right) \ell_{z}+\left(-v_{t 1}-a_{z} \beta_{t 1}\right) \ell_{z}\right]-k_{s 4}^{h}\left[\left(v_{s}-\ell_{z} \beta_{s}+\ell_{x} \alpha_{s}\right) \ell_{z}+\left(-v_{t 2}-a_{z} \beta_{t 2}\right) \ell_{z}\right]=F_{\beta_{s}}
\end{aligned}
$$

e) Para o grau de liberdade de rotação em torno do eixo vertical (yaw) da massa $\mathrm{m}_{\mathrm{s}}$ :

$$
\begin{aligned}
& I_{z s} \ddot{\alpha}_{s}+c_{s 1}^{h}\left(-\dot{v}_{s}+\ell_{z} \dot{\beta}_{s}+\ell_{x} \dot{\alpha}_{s}+\dot{v}_{t 1}+a_{z} \dot{\beta}_{t 1}\right) \ell_{x}-c_{s 2}^{h}\left(-\dot{v}_{s}+\ell_{z} \dot{\beta}_{s}-\ell_{x} \dot{\alpha}_{s}+\dot{v}_{t 2}+a_{z} \dot{\beta}_{t 2}\right) \ell_{x}+ \\
& c_{s 3}^{h}\left(-\dot{v}_{s}+\ell_{z} \dot{\beta}_{s}+\ell_{x} \dot{\alpha}_{s}+\dot{v}_{t 1}+a_{z} \dot{\beta}_{t 1}\right) \ell_{x}-c_{s 4}^{h}\left(-\dot{v}_{s}+\ell_{z} \dot{\beta}_{s}-\ell_{x} \dot{\alpha}_{s}+\dot{v}_{t 2}+a_{z} \dot{\beta}_{t 2}\right) \ell_{x}+ \\
& k_{s 1}^{h}\left(-v_{s}+\ell_{z} \beta_{s}+\ell_{x} \alpha_{s}+v_{t 1}+a_{z} \beta_{t 1}\right) \ell_{x}-k_{s 2}^{h}\left(-v_{s}+\ell_{z} \beta_{s}-\ell_{x} \alpha_{s}+v_{t 2}+a_{z} \beta_{t 2}\right) \ell_{x}+ \\
& k_{s 3}^{h}\left(-v_{s}+\ell_{z} \beta_{s}+\ell_{x} \alpha_{s}+v_{t 1}+a_{z} \beta_{t 1}\right) \ell_{x}-k_{s 4}^{h}\left(-v_{s}+\ell_{z} \beta_{s}-\ell_{x} \alpha_{s}+v_{t 2}+a_{z} \beta_{t 2}\right) \ell_{x}=F_{\alpha_{s}}
\end{aligned}
$$


f) Para o grau de liberdade de deslocamento vertical (bounce) da massa $m_{t 1}$ :

$$
\begin{aligned}
& m_{t 1} \ddot{w}_{t 1}-c_{s 1}^{v}\left(\dot{w}_{s}+\ell_{x} \dot{\theta}_{s}+\ell_{y} \dot{\beta}_{s}-\dot{w}_{t 1}-\ell_{y} \dot{\beta}_{t 1}\right)-c_{s 3}^{v}\left(\dot{w}_{s}+\ell_{x} \dot{\theta}_{s}-\ell_{y} \dot{\beta}_{s}-\dot{w}_{t 1}+\ell_{y} \dot{\beta}_{t 1}\right)- \\
& k_{s 1}^{v}\left(w_{s}+\ell_{x} \theta_{s}+\ell_{y} \beta_{s}-w_{t 1}-\ell_{y} \beta_{t 1}\right)-k_{s 3}^{v}\left(w_{s}+\ell_{x} \theta_{s}-\ell_{y} \beta_{s}-w_{t 1}+\ell_{y} \beta_{t 1}\right)+ \\
& c_{t 1}^{v}\left(\dot{w}_{t 1}+d_{x} \dot{\theta}_{t 1}+d_{y} \dot{\beta}_{t 1}\right)+c_{t 2}^{v}\left(\dot{w}_{t 1}-d_{x} \dot{\theta}_{t 1}+d_{y} \dot{\beta}_{t 1}\right)+c_{t 3}^{v}\left(\dot{w}_{t 1}+d_{x} \dot{\theta}_{t 1}-d_{y} \dot{\beta}_{t 1}\right)+ \\
& c_{t 4}^{v}\left(\dot{w}_{t 1}-d_{x} \dot{\theta}_{t 1}-d_{y} \dot{\beta}_{t 1}\right)+k_{t 1}^{v}\left(w_{t 1}+d_{x} \theta_{t 1}+d_{y} \beta_{t 1}\right)+k_{t 2}^{v}\left(w_{t 1}-d_{x} \theta_{t 1}+d_{y} \beta_{t 1}\right)+ \\
& k_{t 3}^{v}\left(w_{t 1}+d_{x} \theta_{t 1}-d_{y} \beta_{t 1}\right)+k_{t 4}^{v}\left(w_{t 1}-d_{x} \theta_{t 1}-d_{y} \beta_{t 1}\right)=F_{w_{t 1}}
\end{aligned}
$$

g) Para o grau de liberdade de deslocamento transversal (sway) da massa $\mathrm{m}_{\mathrm{t} 1}$ :

$$
\begin{aligned}
& m_{t 1} \ddot{v}_{t 1}-c_{s 1}^{h}\left(\dot{v}_{s}+\ell_{z} \dot{\beta}_{s}-\ell_{x} \dot{\alpha}_{s}-\dot{v}_{t 1}-a_{z} \dot{\beta}_{t 1}\right)-c_{s 3}^{h}\left(\dot{v}_{s}+\ell_{z} \dot{\beta}_{s}-\ell_{x} \dot{\alpha}_{s}-\dot{v}_{t 1}-a_{z} \dot{\beta}_{t 1}\right)- \\
& k_{s 1}^{h}\left(v_{s}+\ell_{z} \beta_{s}-\ell_{x} \alpha_{s}-v_{t 1}-a_{z} \beta_{t 1}\right)-k_{s 3}^{h}\left(v_{s}+\ell_{z} \beta_{s}-\ell_{x} \alpha_{s}-v_{t 1}-a_{z} \beta_{t 1}\right)+c_{t 1}^{h}\left(\dot{v}_{t 1}-b_{z} \dot{\beta}_{t 1}-d_{x} \dot{\alpha}_{t 1}\right)+ \\
& c_{t 2}^{h}\left(\dot{v}_{t 1}-b_{z} \dot{\beta}_{t 1}+d_{x} \dot{\alpha}_{t 1}\right)+c_{t 3}^{h}\left(\dot{v}_{t 1}-b_{z} \dot{\beta}_{t 1}-d_{x} \dot{\alpha}_{t 1}\right)+c_{t 4}^{h}\left(\dot{v}_{t 1}-b_{z} \dot{\beta}_{t 1}+d_{x} \dot{\alpha}_{t 1}\right)+k_{t 1}^{h}\left(v_{t 1}-b_{z} \beta_{t 1}-d_{x} \alpha_{t 1}\right)+ \\
& k_{t 2}^{h}\left(v_{t 1}-b_{z} \beta_{t 1}+d_{x} \alpha_{t 1}\right)+k_{t 3}^{h}\left(v_{t 1}-b_{z} \beta_{t 1}-d_{x} \alpha_{t 1}\right)+k_{t 4}^{h}\left(v_{t 1}-b_{z} \beta_{t 1}+d_{x} \alpha_{t 1}\right)=F_{v_{t 1}}
\end{aligned}
$$

h) Para o grau de liberdade de rotação em torno do eixo transversal (pitch) da massa $m_{\mathrm{t} 1}$ :

$$
\begin{aligned}
& I_{y t 1} \ddot{\theta}_{t 1}+c_{t 1}^{v}\left(\dot{w}_{t 1}+d_{x} \dot{\theta}_{t 1}+d_{y} \dot{\beta}_{t 1}\right) d_{x}-c_{t 2}^{v}\left(\dot{w}_{t 1}-d_{x} \dot{\theta}_{t 1}+d_{y} \dot{\beta}_{t 1}\right) d_{x}+c_{t 3}^{v}\left(\dot{w}_{t 1}+d_{x} \dot{\theta}_{t 1}-d_{y} \dot{\beta}_{t 1}\right) d_{x}- \\
& c_{t 4}^{v}\left(\dot{w}_{t 1}-d_{x} \dot{\theta}_{t 1}-d_{y} \dot{\beta}_{t 1}\right) d_{x}+k_{t 1}^{v}\left(w_{t 1}+d_{x} \theta_{t 1}+d_{y} \beta_{t 1}\right) d_{x}-k_{t 2}^{v}\left(w_{t 1}-d_{x} \theta_{t 1}+d_{y} \beta_{t 1}\right) d_{x}+ \\
& k_{t 3}^{v}\left(w_{t 1}+d_{x} \theta_{t 1}-d_{y} \beta_{t 1}\right) d_{x}-k_{t 4}^{v}\left(w_{t 1}-d_{x} \theta_{t 1}-d_{y} \beta_{t 1}\right) d_{x}=F_{\theta_{t 1}}
\end{aligned}
$$

i) Para o grau de liberdade de rotação em torno do eixo longitudinal (roll) da massa $\mathrm{m}_{\mathrm{t} 1}$ :

$$
\begin{aligned}
& I_{x t 1} \ddot{\beta}_{t 1}-c_{s 1}^{v}\left(\dot{w}_{s}+\ell_{x} \dot{\theta}_{s}+\ell_{y} \dot{\beta}_{s}-\dot{w}_{t 1}-\ell_{y} \dot{\beta}_{t 1}\right) \ell_{y}+c_{s 3}^{v}\left(\dot{w}_{s}+\ell_{x} \dot{\theta}_{s}-\ell_{y} \dot{\beta}_{s}-\dot{w}_{t 1}+\ell_{y} \dot{\beta}_{t 1}\right) \ell_{y}- \\
& k_{s 1}^{v}\left(w_{s}+\ell_{x} \theta_{s}+\ell_{y} \beta_{s}-w_{t 1}-\ell_{y} \beta_{t 1}\right) \ell_{y}+k_{s 3}^{v}\left(w_{s}+\ell_{x} \theta_{s}-\ell_{y} \beta_{s}-w_{t 1}+\ell_{y} \beta_{t 1}\right) \ell_{y}+ \\
& c_{s 1}^{h}\left[\left(-\dot{v}_{s}+\ell_{z} \dot{\beta}_{s}+\ell_{x} \dot{\alpha}_{s}\right) a_{z}+\left(\dot{v}_{t 1}+a_{z} \dot{\beta}_{t 1}\right) a_{z}\right]+c_{s 3}^{h}\left[\left(-\dot{v}_{s}+\ell_{z} \dot{\beta}_{s}+\ell_{x} \dot{\alpha}_{s}\right) a_{z}+\left(\dot{v}_{t 1}+a_{z} \dot{\beta}_{t 1}\right) a_{z}\right]+ \\
& k_{s 1}^{h}\left[\left(-v_{s}+\ell_{z} \beta_{s}+\ell_{x} \alpha_{s}\right) a_{z}+\left(v_{t 1}+a_{z} \beta_{t 1}\right) a_{z}\right]+k_{s 3}^{h}\left[\left(-v_{s}+\ell_{z} \beta_{s}+\ell_{x} \alpha_{s}\right) a_{z}+\left(v_{t 1}+a_{z} \beta_{t 1}\right) a_{z}\right]+ \\
& c_{t 1}^{v}\left(\dot{w}_{t 1}+d_{x} \dot{\theta}_{t 1}+d_{y} \dot{\beta}_{t 1}\right) d_{y}+c_{t 2}^{v}\left(\dot{w}_{t 1}-d_{x} \dot{\theta}_{t 1}+d_{y} \dot{\beta}_{t 1}\right) d_{y}-c_{t 3}^{v}\left(\dot{w}_{t 1}+d_{x} \dot{\theta}_{t 1}-d_{y} \dot{\beta}_{t 1}\right) d_{y}- \\
& c_{t 4}^{v}\left(\dot{w}_{t 1}-d_{x} \dot{\theta}_{t 1}-d_{y} \dot{\beta}_{t 1}\right) d_{y}+k_{t 1}^{v}\left(w_{t 1}+d_{x} \theta_{t 1}+d_{y} \beta_{t 1}\right) d_{y}+k_{t 2}^{v}\left(w_{t 1}-d_{x} \theta_{t 1}+d_{y} \beta_{t 1}\right) d_{y}- \\
& k_{t 3}^{v}\left(w_{t 1}+d_{x} \theta_{t 1}-d_{y} \beta_{t 1}\right) d_{y}-k_{t 4}^{v}\left(w_{t 1}-d_{x} \theta_{t 1}-d_{y} \beta_{t 1}\right) d_{y}-c_{t 1}^{h}\left(\dot{v}_{t 1}-b_{z} \dot{\beta}_{t 1}-d_{x} \dot{\alpha}_{t 1}\right) b_{z}- \\
& c_{t 2}^{h}\left(\dot{v}_{t 1}-b_{z} \dot{\beta}_{t 1}+d_{x} \dot{\alpha}_{t 1}\right) b_{z}-c_{t 3}^{h}\left(\dot{v}_{t 1}-b_{z} \dot{\beta}_{t 1}-d_{x} \dot{\alpha}_{t 1}\right) b_{z}-c_{t 4}^{h}\left(\dot{v}_{t 1}-b_{z} \dot{\beta}_{t 1}+d_{x} \dot{\alpha}_{t 1}\right) b_{z}- \\
& k_{t 1}^{h}\left(v_{t 1}-b_{z} \beta_{t 1}-d_{x} \alpha_{t 1}\right) b_{z}-k_{t 2}^{h}\left(v_{t 1}-b_{z} \beta_{t 1}+d_{x} \alpha_{t 1}\right) b_{z}-k_{t 3}^{h}\left(v_{t 1}-b_{z} \beta_{t 1}-d_{x} \alpha_{t 1}\right) b_{z}- \\
& k_{t 4}^{h}\left(v_{t 1}-b_{z} \beta_{t 1}+d_{x} \alpha_{t 1}\right) b_{z}=F_{\beta_{t 1}}
\end{aligned}
$$


j) Para o grau de liberdade de rotação em torno do eixo vertical (yaw) da massa $m_{t 1}$ :

$I_{z t 1} \ddot{\alpha}_{t 1}+c_{t 1}^{h}\left(-\dot{v}_{t 1}+b_{z} \dot{\beta}_{t 1}+d_{x} \dot{\alpha}_{t 1}\right) d_{x}-c_{t 2}^{h}\left(-\dot{v}_{t 1}+b_{z} \dot{\beta}_{t 1}-d_{x} \dot{\alpha}_{t 1}\right) d_{x}+c_{t 3}^{h}\left(-\dot{v}_{t 1}+b_{z} \dot{\beta}_{t 1}+d_{x} \dot{\alpha}_{t 1}\right) d_{x}-$ $c_{t 4}^{h}\left(-\dot{v}_{t 1}+b_{z} \dot{\beta}_{t 1}-d_{x} \dot{\alpha}_{t 1}\right) d_{x}+k_{t 1}^{h}\left(-v_{t 1}+b_{z} \beta_{t 1}+d_{x} \alpha_{t 1}\right) d_{x}-k_{t 2}^{h}\left(-v_{t 1}+b_{z} \beta_{t 1}-d_{x} \alpha_{t 1}\right) d_{x}+$ $k_{t 3}^{h}\left(-v_{t 1}+b_{z} \beta_{t 1}+d_{x} \alpha_{t 1}\right) d_{x}-k_{t 4}^{h}\left(-v_{t 1}+b_{z} \beta_{t 1}-d_{x} \alpha_{t 1}\right) d_{x}=F_{\alpha t 1}$

k) Para o grau de liberdade de deslocamento vertical (bounce) da massa $\mathrm{m}_{\mathrm{t} 2}$ :

$$
\begin{aligned}
& m_{t 2} \ddot{w}_{t 2}-c_{s 2}^{v}\left(\dot{w}_{s}-\ell_{x} \dot{\theta}_{s}+\ell_{y} \dot{\beta}_{s}-\dot{w}_{t 2}-\ell_{y} \dot{\beta}_{t 2}\right)-c_{s 4}^{v}\left(\dot{w}_{s}-\ell_{x} \dot{\theta}_{s}-\ell_{y} \dot{\beta}_{s}-\dot{w}_{t 2}+\ell_{y} \dot{\beta}_{t 2}\right)- \\
& k_{s 2}^{v}\left(w_{s}-\ell_{x} \theta_{s}+\ell_{y} \beta_{s}-w_{t 2}-\ell_{y} \beta_{t 2}\right)-k_{s 4}^{v}\left(w_{s}-\ell_{x} \theta_{s}-\ell_{y} \beta_{s}-w_{t 2}+\ell_{y} \beta_{t 2}\right)+ \\
& c_{t 5}^{v}\left(\dot{w}_{t 2}+d_{x} \dot{\theta}_{t 2}+d_{y} \dot{\beta}_{t 2}\right)+c_{t 6}^{v}\left(\dot{w}_{t 2}-d_{x} \dot{\theta}_{t 2}+d_{y} \dot{\beta}_{t 2}\right)+c_{t 7}^{v}\left(\dot{w}_{t 2}+d_{x} \dot{\theta}_{t 2}-d_{y} \dot{\beta}_{t 2}\right)+ \\
& c_{t 8}^{v}\left(\dot{w}_{t 2}-d_{x} \dot{\theta}_{t 2}-d_{y} \dot{\beta}_{t 2}\right)+k_{t 5}^{v}\left(w_{t 2}+d_{x} \theta_{t 2}+d_{y} \beta_{t 2}\right)+k_{t 6}^{v}\left(w_{t 2}-d_{x} \theta_{t 2}+d_{y} \beta_{t 2}\right)+ \\
& k_{t 7}^{v}\left(w_{t 2}+d_{x} \theta_{t 2}-d_{y} \beta_{t 2}\right)+k_{t 8}^{v}\left(w_{t 2}-d_{x} \theta_{t 2}-d_{y} \beta_{t 2}\right)=F_{w_{t 2}}
\end{aligned}
$$

I) Para o grau de liberdade de deslocamento transversal (sway) da massa $\mathrm{m}_{\mathrm{t} 2}$ :

$$
\begin{aligned}
& m_{t 2} \ddot{v}_{t 2}-c_{s 2}^{h}\left(\dot{v}_{s}-\ell_{z} \dot{\beta}_{s}+\ell_{x} \dot{\alpha}_{s}-\dot{v}_{t 2}-a_{z} \dot{\beta}_{t 2}\right)-c_{s 4}^{h}\left(\dot{v}_{s}-\ell_{z} \dot{\beta}_{s}+\ell_{x} \dot{\alpha}_{s}-\dot{v}_{t 2}-a_{z} \dot{\beta}_{t 2}\right)- \\
& k_{s 2}^{h}\left(v_{s}-\ell_{z} \beta_{s}+\ell_{x} \alpha_{s}-v_{t 2}-a_{z} \beta_{t 2}\right)-k_{s 4}^{h}\left(v_{s}-\ell_{z} \beta_{s}+\ell_{x} \alpha_{s}-v_{t 2}-a_{z} \beta_{t 2}\right)+c_{t 5}^{h}\left(\dot{v}_{t 2}-b_{z} \dot{\beta}_{t 2}-d_{x} \dot{\alpha}_{t 2}\right)+ \\
& c_{t 6}^{h}\left(\dot{v}_{t 2}-b_{z} \dot{\beta}_{t 2}+d_{x} \dot{\alpha}_{t 2}\right)+c_{t 7}^{h}\left(\dot{v}_{t 2}-b_{z} \dot{\beta}_{t 2}-d_{x} \dot{\alpha}_{t 2}\right)+c_{t 8}^{h}\left(\dot{v}_{t 2}-b_{z} \dot{\beta}_{t 2}+d_{x} \dot{\alpha}_{t 2}\right)+k_{t 5}^{h}\left(v_{t 2}-b_{z} \beta_{t 2}-d_{x} \alpha_{t 2}\right)+ \\
& k_{t 6}^{h}\left(v_{t 2}-b_{z} \beta_{t 2}+d_{x} \alpha_{t 2}\right)+k_{t 7}^{h}\left(v_{t 2}-b_{z} \beta_{t 2}-d_{x} \alpha_{t 2}\right)+k_{t 8}^{h}\left(v_{t 2}-b_{z} \beta_{t 2}+d_{x} \alpha_{t 2}\right)=F_{v_{t 2}}
\end{aligned}
$$

m) Para o grau de liberdade de rotação em torno do eixo transversal (pitch) da massa $\mathrm{m}_{\mathrm{t} 2}$ :

$$
\begin{aligned}
& I_{y t 2} \ddot{\theta}_{t 2}+c_{t 5}^{v}\left(\dot{w}_{t 2}+d_{x} \dot{\theta}_{t 2}+d_{y} \dot{\beta}_{t 2}\right) d_{x}-c_{t 6}^{v}\left(\dot{w}_{t 2}-d_{x} \dot{\theta}_{t 2}+d_{y} \dot{\beta}_{t 2}\right) d_{x}+c_{t 7}^{v}\left(\dot{w}_{t 2}+d_{x} \dot{\theta}_{t 2}-d_{y} \dot{\beta}_{t 2}\right) d_{x}- \\
& c_{t 8}^{v}\left(\dot{w}_{t 2}-d_{x} \dot{\theta}_{t 2}-d_{y} \dot{\beta}_{t 2}\right) d_{x}+k_{t 5}^{v}\left(w_{t 2}+d_{x} \theta_{t 2}+d_{y} \beta_{t 2}\right) d_{x}-k_{t 6}^{v}\left(w_{t 2}-d_{x} \theta_{t 2}+d_{y} \beta_{t 2}\right) d_{x}+ \\
& k_{t 7}^{v}\left(w_{t 2}+d_{x} \theta_{t 2}-d_{y} \beta_{t 2}\right) d_{x}-k_{t 8}^{v}\left(w_{t 2}-d_{x} \theta_{t 2}-d_{y} \beta_{t 2}\right) d_{x}=F_{\theta_{t 2}}
\end{aligned}
$$


n) Para o grau de liberdade de rotação em torno do eixo longitudinal (roll) da massa $\mathrm{m}_{\mathrm{t} 2}$ :

$$
\begin{aligned}
& I_{x t 2} \ddot{\beta}_{t 2}-c_{s 2}^{v}\left(\dot{w}_{s}-\ell_{x} \dot{\theta}_{s}+\ell_{y} \dot{\beta}_{s}-\dot{w}_{t 2}-\ell_{y} \dot{\beta}_{t 2}\right) \ell_{y}+c_{s 4}^{v}\left(\dot{w}_{s}-\ell_{x} \dot{\theta}_{s}-\ell_{y} \dot{\beta}_{s}-\dot{w}_{t 2}+\ell_{y} \dot{\beta}_{t 2}\right) \ell_{y}- \\
& k_{s 2}^{v}\left(w_{s}-\ell_{x} \theta_{s}+\ell_{y} \beta_{s}-w_{t 2}-\ell_{y} \beta_{t 2}\right) \ell_{y}+k_{s 4}^{v}\left(w_{s}-\ell_{x} \theta_{s}-\ell_{y} \beta_{s}-w_{t 2}+\ell_{y} \beta_{t 2}\right) \ell_{y}+ \\
& c_{s 2}^{h}\left[\left(-\dot{v}_{s}+\ell_{z} \dot{\beta}_{s}-\ell_{x} \dot{\alpha}_{s}\right) a_{z}+\left(\dot{v}_{t 2}+a_{z} \dot{\beta}_{t 2}\right) a_{z}\right]+c_{s 4}^{h}\left[\left(-\dot{v}_{s}+\ell_{z} \dot{\beta}_{s}-\ell_{x} \dot{\alpha}_{s}\right) a_{z}+\left(\dot{v}_{t 2}+a_{z} \dot{\beta}_{t 2}\right) a_{z}\right]+ \\
& k_{s 2}^{h}\left[\left(-v_{s}+\ell_{z} \beta_{s}-\ell_{x} \alpha_{s}\right) a_{z}+\left(v_{t 2}+a_{z} \beta_{t 2}\right) a_{z}\right]+k_{s 4}^{h}\left[\left(-v_{s}+\ell_{z} \beta_{s}-\ell_{x} \alpha_{s}\right) a_{z}+\left(v_{t 2}+a_{z} \beta_{t 2}\right) a_{z}\right]+ \\
& c_{t 5}^{v}\left(\dot{w}_{t 2}+d_{x} \dot{\theta}_{t 2}+d_{y} \dot{\beta}_{t 2}\right) d_{y}+c_{t 6}^{v}\left(\dot{w}_{t 2}-d_{x} \dot{\theta}_{t 2}+d_{y} \dot{\beta}_{t 2}\right) d_{y}-c_{t 7}^{v}\left(\dot{w}_{t 2}+d_{x} \dot{\theta}_{t 2}-d_{y} \dot{\beta}_{t 2}\right) d_{y}- \\
& c_{t 8}^{v}\left(\dot{w}_{t 2}-d_{x} \dot{\theta}_{t 2}-d_{y} \dot{\beta}_{t 2}\right) d_{y}+k_{t 5}^{v}\left(w_{t 2}+d_{x} \theta_{t 2}+d_{y} \beta_{t 2}\right) d_{y}+k_{t 6}^{v}\left(w_{t 2}-d_{x} \theta_{t 2}+d_{y} \beta_{t 2}\right) d_{y}- \\
& k_{t 7}^{v}\left(w_{t 2}+d_{x} \theta_{t 2}-d_{y} \beta_{t 2}\right) d_{y}-k_{t 8}^{v}\left(w_{t 2}-d_{x} \theta_{t 2}-d_{y} \beta_{t 2}\right) d_{y}-c_{t 5}^{h}\left(\dot{v}_{t 2}-b_{z} \dot{\beta}_{t 2}-d_{x} \dot{\alpha}_{t 2}\right) b_{z}- \\
& c_{t 6}^{h}\left(\dot{v}_{t 2}-b_{z} \dot{\beta}_{t 2}+d_{x} \dot{\alpha}_{t 2}\right) b_{z}-c_{t 7}^{h}\left(\dot{v}_{t 2}-b_{z} \dot{\beta}_{t 2}-d_{x} \dot{\alpha}_{t 2}\right) b_{z}-c_{t 8}^{h}\left(\dot{v}_{t 2}-b_{z} \dot{\beta}_{t 2}+d_{x} \dot{\alpha}_{t 2}\right) b_{z}- \\
& k_{t 5}^{h}\left(v_{t 2}-b_{z} \beta_{t 2}-d_{x} \alpha_{t 2}\right) b_{z}-k_{t 6}^{h}\left(v_{t 2}-b_{z} \beta_{t 2}+d_{x} \alpha_{t 2}\right) b_{z}-k_{t 7}^{h}\left(v_{t 2}-b_{z} \beta_{t 2}-d_{x} \alpha_{t 2}\right) b_{z}- \\
& k_{t 8}^{h}\left(v_{t 2}-b_{z} \beta_{t 2}+d_{x} \alpha_{t 2}\right) b_{z}=F_{\beta_{t 2}}
\end{aligned}
$$

o) Para o grau de liberdade de rotação em torno do eixo vertical (yaw) da massa $m_{\mathrm{t} 2}:$

$$
\begin{aligned}
& I_{z t 2} \ddot{\alpha}_{t 2}+c_{t 5}^{h}\left(-\dot{v}_{t 2}+b_{z} \dot{\beta}_{t 2}+d_{x} \dot{\alpha}_{t 2}\right) d_{x}-c_{t 6}^{h}\left(-\dot{v}_{t 2}+b_{z} \dot{\beta}_{t 2}-d_{x} \dot{\alpha}_{t 2}\right) d_{x}+c_{t 7}^{h}\left(-\dot{v}_{t 2}+b_{z} \dot{\beta}_{t 2}+d_{x} \dot{\alpha}_{t 2}\right) d_{x}- \\
& c_{t 8}^{h}\left(-\dot{v}_{t 2}+b_{z} \dot{\beta}_{t 2}-d_{x} \dot{\alpha}_{t 2}\right) d_{x}+k_{t 5}^{h}\left(-v_{t 2}+b_{z} \beta_{t 2}+d_{x} \alpha_{t 2}\right) d_{x}-k_{t 6}^{h}\left(-v_{t 2}+b_{z} \beta_{t 2}-d_{x} \alpha_{t 2}\right) d_{x}+ \\
& k_{t 7}^{h}\left(-v_{t 2}+b_{z} \beta_{t 2}+d_{x} \alpha_{t 2}\right) d_{x}-k_{t 8}^{h}\left(-v_{t 2}+b_{z} \beta_{t 2}-d_{x} \alpha_{t 2}\right) d_{x}=F_{\alpha t 2}
\end{aligned}
$$

Em notação matricial, é possível escrever as equações da seguinte forma:

$[M]\{\ddot{U}\}+[C]\{\dot{U}\}+[K]\{U\}=\{F\}$,

com:

$\{U\}^{T}=\left[\begin{array}{lllllllllllllll}w_{s} & v_{s} & \theta_{s} & \beta_{s} & \alpha_{s} & w_{t 1} & v_{t 1} & \theta_{t 1} & \beta_{t l} & \alpha_{t 1} & w_{t 2} & v_{t 2} & \theta_{t 2} & \beta_{t 2} & \alpha_{t 2}\end{array}\right]$

$\{\dot{U}\}^{T}=\left[\begin{array}{lllllllllllllll}\dot{w}_{s} & \dot{v}_{s} & \dot{\theta}_{s} & \dot{\beta}_{s} & \dot{\alpha}_{s} & \dot{w}_{t l} & \dot{v}_{t l} & \dot{\theta}_{t l} & \dot{\beta}_{t 1} & \dot{\alpha}_{t 1} & \dot{w}_{t 2} & \dot{v}_{t 2} & \dot{\theta}_{t 2} & \dot{\beta}_{t 2} & \dot{\alpha}_{t 2}\end{array}\right]$

$\{\ddot{U}\}^{T}=\left[\begin{array}{lllllllllllllll}\ddot{w}_{s} & \ddot{v}_{s} & \ddot{\theta}_{s} & \ddot{\beta}_{s} & \ddot{\alpha}_{s} & \ddot{w}_{t l} & \ddot{v}_{t l} & \ddot{\theta}_{t l} & \ddot{\beta}_{t l} & \ddot{\alpha}_{t l} & \ddot{w}_{t 2} & \ddot{v}_{t 2} & \ddot{\theta}_{t 2} & \ddot{\beta}_{t 2} & \ddot{\alpha}_{t 2}\end{array}\right]$

e

$[M]=\left\lfloor M_{i, j}\right\rfloor$

onde:

$\begin{array}{lllll}M_{1,1}=m_{s} & M_{2,2}=m_{s} & M_{3,3}=I_{y s} & M_{4,4}=I_{x s} & M_{5,5}=I_{z s} \\ M_{6,6}=m_{t 1} & M_{7,7}=m_{t 1} & M_{8,8}=I_{y t 1} & M_{9,9}=I_{x t 1} & M_{10,10}=I_{z t 1} \\ M_{11,11}=m_{t 2} & M_{12,12}=m_{t 2} & M_{13,13}=I_{y t 2} & M_{14,14}=I_{x t 2} & M_{15,15}=I_{z t 2}\end{array}$




$$
\begin{aligned}
& M_{i, j}=0 \text { para } i \neq j \\
& {[C]=\left\lfloor C_{i, j}\right\rfloor}
\end{aligned}
$$

onde:

$$
\begin{aligned}
& C_{1,1}=\sum_{i=1}^{4} c_{s i}^{v} \\
& C_{1,2}=C_{2,1}=0 \\
& C_{1,3}=C_{3,1}=\sum_{i=1}^{4}(-1)^{i+1} c_{s i}^{v} \ell_{x} \\
& C_{1,4}=C_{4,1}=\sum_{i=1}^{2} c_{s i}^{v} \ell_{y}-\sum_{i=3}^{4} c_{s i}^{v} \ell_{y} \\
& C_{1,5}=C_{5,1}=0 \\
& C_{1,6}=C_{6,1}=-\sum_{i=1}^{2} c_{s(2 i-1)}^{v} \\
& C_{1,7}=C_{7,1}=0 \\
& C_{1,8}=C_{8,1}=0 \\
& C_{1,9}=C_{9,1}=\sum_{i=1}^{2}(-1)^{i} c_{s(2 i-1)}^{v} \ell_{y} \\
& C_{1,10}=C_{10,1}=0 \\
& C_{1,11}=C_{11,1}=-\sum_{i=1}^{2} c_{s(2 i)}^{v} \\
& C_{1,12}=C_{12,1}=0 \\
& C_{1,13}=C_{13,1}=0 \\
& C_{1,14}=C_{14,1}=\sum_{i=1}^{2}(-1)^{i} c_{s(2 i)}^{v} \ell_{y} \\
& C_{1,15}=C_{15,1}=0 \\
& C_{2,2}=\sum_{i=1}^{4} c_{s i}^{h} \\
& C_{2,3}=C_{3,2}=0 \\
& C_{2,4}=C_{4,2}=-\sum_{i=1}^{4} c_{s i}^{h} \ell_{z} \\
& C_{2,5}=C_{5,2}=\sum_{i=1}^{4}(-1)^{i} c_{s i}^{h} \ell_{x} \\
& C_{2,6}=C_{6,2}=0 \\
& C_{2,7}=C_{7,2}=-\sum_{i=1}^{2} c_{s(2 i-1)}^{h} \\
& C_{2,8}=C_{8,2}=0 \\
& C_{2,9}=C_{9,2}=-\sum_{i=1}^{2} c_{s(2 i-1)}^{h} a_{z} \\
& C_{2,10}=C_{10,2}=0 \\
& C_{2,11}=C_{11,2}=0 \\
& C_{2,12}=C_{12,2}=-\sum_{i=1}^{2} c_{s(2 i)}^{h} \\
& C_{2,13}=C_{13,2}=0 \\
& C_{2,14}=C_{14,2}=-\sum_{i=1}^{2} c_{s(2 i)}^{h} a_{z} \\
& C_{2,15}=C_{15,2}=0 \\
& C_{3,3}=\sum_{i=1}^{4} c_{s i}^{v} \ell_{x}^{2} \\
& C_{3,4}=C_{4,3}=\sum_{i=1}^{2}(-1)^{i+1} c_{s i}^{v} \ell_{x} \ell_{y}+\sum_{i=3}^{4}(-1)^{i} c_{s i}^{v} \ell_{x} \ell_{y} \\
& C_{3,5}=C_{5,3}=0
\end{aligned}
$$




$$
\begin{aligned}
& C_{3,6}=C_{6,3}=-\sum_{i=1}^{2} c_{s(2 i-1)}^{v} \ell_{x} \\
& C_{3,7}=C_{7,3}=0 \\
& C_{3,8}=C_{8,3}=0 \\
& C_{3,9}=C_{9,3}=\sum_{i=1}^{2}(-1)^{i} c_{s(2 i-1)}^{v} \ell_{x} \ell_{y} \\
& C_{3,10}=C_{10,3}=0 \\
& C_{3,11}=C_{11,3}=\sum_{i=1}^{2} c_{s(2 i)}^{v} \ell_{x} \\
& C_{3,12}=C_{12,3}=0 \\
& C_{3,13}=C_{13,3}=0 \\
& C_{3,14}=C_{14,3}=\sum_{i=1}^{2}(-1)^{i+1} c_{s(2 i)}^{v} \ell_{x} \ell_{y} \\
& C_{3,15}=C_{15,3}=0 \\
& C_{4,4}=\sum_{i=1}^{4} c_{s i}^{v} \ell_{y}{ }^{2}+\sum_{i=1}^{4} c_{s i}^{h} \ell_{z}^{2} \\
& C_{4,5}=C_{5,4}=\sum_{i=1}^{4}(-1)^{i+1} c_{s i}^{h} \ell_{x} \ell_{z} \\
& C_{4,6}=C_{6,4}=\sum_{i=1}^{2}(-1)^{i} c_{s(2 i-1)}^{v} \ell_{y} \\
& C_{4,7}=C_{7,4}=-\sum_{i=1}^{2} c_{s(2 i-1)}^{h} \ell_{z} \\
& C_{4,8}=C_{8,4}=0 \\
& C_{4,9}=C_{9,4}=-\sum_{i=1}^{2} c_{s(2 i-1)}^{v} \ell_{y}{ }^{2}+\sum_{i=1}^{2} c_{s(2 i-1)}^{h} a_{z} \ell_{z} \\
& C_{4,10}=C_{10,4}=0 \\
& C_{4,11}=C_{11,4}=\sum_{i=1}^{2}(-1)^{i} c_{s(2 i)}^{v} \ell_{y} \\
& C_{4,12}=C_{12,4}=\sum_{i=1}^{2} c_{s(2 i)}^{h} \ell_{z} \\
& C_{4,13}=C_{13,4}=0 \\
& C_{4,14}=C_{14,4}=-\sum_{i=1}^{2} c_{s(2 i)}^{v} \ell_{y}{ }^{2}+\sum_{i=1}^{2} c_{s(2 i)}^{h} a_{z} \ell_{z} \\
& C_{4,15}=C_{15,4}=0 \\
& C_{5,5}=\sum_{i=1}^{4} c_{s i}^{h} \ell_{x}^{2} \\
& C_{5,6}=C_{6,5}=0 \\
& C_{5,7}=C_{7,5}=\sum_{i=1}^{2} c_{s(2 i-1)}^{h} \ell_{x} \\
& C_{5,8}=C_{8,5}=0 \\
& C_{5,9}=C_{9,5}=\sum_{i=1}^{2} c_{s(2 i-1)}^{h} a_{z} \ell_{x} \\
& C_{5,10}=C_{10,5}=0 \\
& C_{5,11}=C_{11,5}=0 \\
& C_{5,12}=C_{12,5}=-\sum_{i=1}^{2} c_{s(2 i)}^{h} \ell_{x} \\
& C_{5,13}=C_{13,5}=0 \\
& C_{5,14}=-\sum_{i=1}^{2} c_{s(2 i)}^{h} a_{z} \ell_{x} \\
& C_{5,15}=C_{15,5}=0 \\
& C_{6,6}=\sum_{i=1}^{2} c_{s(2 i-1)}^{v}+\sum_{i=1}^{4} c_{t i}^{v} \\
& C_{6,7}=C_{7,6}=0 \\
& C_{6,8}=\sum_{i=1}^{4}(-1)^{i+1} c_{t i}^{v} d_{x}
\end{aligned}
$$




$$
\begin{aligned}
& C_{6,9}=\sum_{i=1}^{2}(-1)^{i+1} c_{s(2 i-1)}^{v} \ell_{y}+\sum_{i=1}^{2} c_{t i}^{v} d_{y}-\sum_{i=3}^{4} c_{t i}^{v} d_{y} \\
& C_{6,10}=C_{10,6}=0 \\
& C_{6,11}=C_{11,6}=0 \\
& C_{6,12}=C_{12,6}=0 \\
& C_{6,13}=C_{13,6}=0 \\
& C_{6,14}=C_{14,6}=0 \\
& C_{6,15}=C_{15,6}=0 \\
& C_{7,7}=\sum_{i=1}^{2} c_{s(2 i-1)}^{h}+\sum_{i=1}^{4} c_{t i}^{h} \\
& C_{7,8}=C_{8,7}=0 \\
& C_{7,9}=\sum_{i=1}^{2} c_{s(2 i-1)}^{h} a_{z}-\sum_{i=1}^{4} c_{t i}^{h} b_{z} \\
& C_{7,10}=\sum_{i=1}^{4}(-1)^{i} c_{t i}^{h} d_{x} \\
& C_{7,11}=C_{11,7}=0 \\
& C_{7,12}=C_{12,7}=0 \\
& C_{7,13}=C_{13,7}=0 \\
& C_{7,14}=C_{14,7}=0 \\
& C_{7,15}=C_{15,7}=0 \\
& C_{8,8}=\sum_{i=1}^{4} c_{t i}^{v} d_{x}^{2} \\
& C_{8,9}=C_{9,8}=\sum_{i=1}^{2}(-1)^{i+1} c_{t i}^{v} d_{x} d_{y}+\sum_{i=3}^{4}(-1)^{i} c_{t i}^{v} d_{x} d_{y} \\
& C_{8,10}=C_{10,8}=0 \\
& C_{8,11}=C_{11,8}=0 \\
& C_{8,12}=C_{12,8}=0 \\
& C_{8,13}=C_{13,8}=0 \\
& C_{8,14}=C_{14,8}=0 \\
& C_{8,15}=C_{15,8}=0 \\
& C_{9,9}=\sum_{i=1}^{2} c_{s(2 i-1)}^{v} l_{y}{ }^{2}+\sum_{i=1}^{2} c_{s(2 i-1)}^{h} a_{z}{ }^{2}+\sum_{i=1}^{4} c_{t i}^{v} d_{y}{ }^{2}+\sum_{i=1}^{4} c_{t i}^{h} b_{z}{ }^{2} \\
& C_{9,10}=C_{10,9}=\sum_{i=1}^{4}(-1)^{i+1} c_{t i}^{h} d_{x} b_{z} \\
& C_{9,11}=C_{11,9}=0 \\
& C_{9,12}=C_{12,9}=0 \\
& C_{9,13}=C_{13,9}=0 \\
& C_{9,14}=C_{14,9}=0 \\
& C_{9,15}=C_{15,9}=0 \\
& C_{10,10}=\sum_{i=1}^{4} c_{t i}^{h} d_{x}{ }^{2} \\
& C_{10,11}=C_{11,10}=0 \\
& C_{10,12}=C_{12,10}=0 \\
& C_{10,13}=C_{13,10}=0 \\
& C_{10,14}=C_{14,10}=0 \\
& C_{10,15}=C_{15,10}=0 \\
& C_{11,11}=\sum_{i=1}^{2} c_{s(2 i)}^{v}+\sum_{i=5}^{8} c_{t i}^{v} \\
& C_{11,12}=C_{12,11}=0 \\
& C_{11,13}=C_{13,11}=\sum_{i=5}^{8}(-1)^{i+1} c_{t i}^{v} d_{x} \\
& C_{11,14}=C_{14,11}=\sum_{i=1}^{2}(-1)^{i+1} c_{s(2 i)}^{v} \ell_{y}+\sum_{i=5}^{6} c_{t i}^{v} d_{y}-\sum_{i=7}^{8} c_{t i}^{v} d_{y} \\
& C_{11,15}=C_{15,11}=0
\end{aligned}
$$




$$
\begin{aligned}
& C_{12,12}=\sum_{i=1}^{2} c_{s(2 i)}^{h}+\sum_{i=5}^{8} c_{t i}^{h} \\
& C_{12,13}=C_{13,12}=0 \\
& C_{12,14}=C_{14,12}=\sum_{i=1}^{2} c_{s(2 i)}^{h} a_{z}-\sum_{i=5}^{8} c_{t i}^{h} b_{z} \\
& C_{12,15}=C_{15,12}=\sum_{i=5}^{8}(-1)^{i} c_{t i}^{h} d_{x} \\
& C_{13,13}=\sum_{i=5}^{8} c_{t i}^{v} d_{x}^{2} \\
& C_{13,14}=C_{14,13}=\sum_{i=5}^{6}(-1)^{i+1} c_{t i}^{h} d_{x} d_{y}+\sum_{i=7}^{8}(-1)^{i} c_{t i}^{h} d_{x} d_{y} \\
& C_{13,15}=C_{15,13}=0 \\
& C_{14,14}=\sum_{i=1}^{2} c_{s(2 i)}^{v} \ell_{y}{ }^{2}+\sum_{i=1}^{2} c_{s(2 i)}^{h} a_{z}{ }^{2}+\sum_{i=5}^{8} c_{t i}^{v} d_{y}{ }^{2}+\sum_{i=5}^{8} c_{t i}^{h} b_{z}^{2} \\
& C_{14,15}=C_{15,14}=\sum_{i=5}^{8}(-1)^{i+1} c_{t i}^{h} b_{z} d_{x} \\
& C_{15,15}=\sum_{i=5}^{8} c_{t i}^{h} d_{x}^{2} \\
& {[K]=\left\lfloor K_{i, j}\right\rfloor} \\
& K_{1,1}=\sum_{i=1}^{4} k_{s i}^{v} \\
& K_{1,3}=K_{3,1}=\sum_{i=1}^{4}(-1)^{i+1} k_{s i}^{v} \ell_{x} \\
& K_{1,5}=K_{5,1}=0 \\
& K_{1,7}=K_{7,1}=0 \\
& K_{1,9}=K_{9,1}=\sum_{i=1}^{2}(-1)^{i} k_{s(2 i-1)}^{v} \ell_{y} \\
& K_{1,11}=K_{11,1}=-\sum_{i=1}^{2} k_{s(2 i)}^{v} \\
& K_{1,13}=K_{13,1}=0 \\
& K_{1,15}=K_{15,1}=0 \\
& K_{2,3}=K_{3,2}=0 \\
& K_{2,5}=K_{5,2}=\sum_{i=1}^{4}(-1)^{i} k_{s i}^{h} \ell_{x} \\
& K_{1,2}=K_{2,1}=0 \\
& K_{1,4}=K_{4,1}=\sum_{i=1}^{2} k_{s i}^{v} \ell_{y}-\sum_{i=3}^{4} k_{s i}^{v} \ell_{y} \\
& K_{1,6}=K_{6,1}=-\sum_{i=1}^{2} k_{s(2 i-1)}^{v} \\
& K_{1,8}=K_{8,1}=0 \\
& K_{1,10}=K_{10,1}=0 \\
& K_{1,12}=K_{12,1}=0 \\
& K_{1,14}=K_{14,1}=\sum_{i=1}^{2}(-1)^{i} k_{s(2 i)}^{v} \ell_{y} \\
& K_{2,2}=\sum_{i=1}^{4} k_{s i}^{h} \\
& k_{2,4}=k_{4,2}=-\sum_{i=1}^{4} k_{s i}^{h} \ell_{z} \\
& K_{2,6}=K_{6,2}=0
\end{aligned}
$$




$$
\begin{aligned}
& K_{2,7}=K_{7,2}=-\sum_{i=1}^{2} k_{s(2 i-1)}^{h} \\
& K_{2,8}=K_{8,2}=0 \\
& K_{2,9}=K_{9,2}=-\sum_{i=1}^{2} k_{s(2 i-1)}^{h} a_{z} \\
& K_{2,10}=K_{10,2}=0 \\
& K_{2,11}=K_{11,2}=0 \\
& K_{2,12}=K_{12,2}=-\sum_{i=1}^{2} k_{s(2 i)}^{h} \\
& K_{2,13}=K_{13,2}=0 \\
& K_{2,14}=K_{14,2}=-\sum_{i=1}^{2} k_{s(2 i)}^{h} a_{z} \\
& K_{2,15}=K_{15,2}=0 \\
& K_{3,3}=\sum_{i=1}^{4} k_{s i}^{v} \ell_{x}^{2} \\
& K_{3,4}=K_{4,3}=\sum_{i=1}^{2}(-1)^{i+1} k_{s i}^{v} \ell_{x} \ell_{y}+\sum_{i=3}^{4}(-1)^{i} k_{s i}^{v} \ell_{x} \ell_{y} \\
& K_{3,5}=K_{5,3}=0 \\
& K_{3,6}=K_{6,3}=-\sum_{i=1}^{2} k_{s(2 i-1)}^{v} \ell_{x} \\
& K_{3,7}=K_{7,3}=0 \\
& K_{3,8}=K_{8,3}=0 \\
& K_{3,9}=K_{9,3}=\sum_{i=1}^{2}(-1)^{i} k_{s(2 i-1)}^{v} \ell_{x} \ell_{y} \\
& K_{3,10}=K_{10,3}=0 \\
& K_{3,11}=K_{11,3}=\sum_{i=1}^{2} k_{s(2 i)}^{v} \ell_{x} \\
& K_{3,12}=K_{12,3}=0 \\
& K_{3,13}=K_{13,3}=0 \\
& K_{3,14}=K_{14,3}=\sum_{i=1}^{2}(-1)^{i+1} k_{s(2 i)}^{v} \ell_{x} \ell_{y} \\
& K_{3,15}=K_{15,3}=0 \\
& K_{4,4}=\sum_{i=1}^{4} k_{s i}^{v} \ell_{y}^{2}+\sum_{i=1}^{4} k_{s i}^{h} \ell_{z}^{2} \\
& K_{4,5}=K_{5,4}=\sum_{i=1}^{4}(-1)^{i+1} k_{s i}^{h} \ell_{x} \ell_{z} \\
& K_{4,6}=K_{6,4}=\sum_{i=1}^{2}(-1)^{i} k_{s(2 i-1)}^{v} \ell_{y} \\
& K_{4,7}=K_{7,4}=-\sum_{i=1}^{2} k_{s(2 i-1)}^{h} \ell_{z} \\
& K_{4,8}=K_{8,4}=0 \\
& K_{4,9}=K_{9,4}=-\sum_{i=1}^{2} k_{s(2 i-1)}^{v} \ell_{y}{ }^{2}+\sum_{i=1}^{2} k_{s(2 i-1)}^{h} a_{z} \ell_{z} \\
& K_{4,10}=K_{10,4}=0 \\
& K_{4,11}=K_{11,4}=\sum_{i=1}^{2}(-1)^{i} k_{s(2 i)}^{v} \ell_{y} \\
& K_{4,12}=K_{12,4}=\sum_{i=1}^{2} k_{s(2 i)}^{h} \ell_{z} \\
& K_{4,13}=K_{13,4}=0 \\
& K_{4,14}=K_{14,4}=-\sum_{i=1}^{2} k_{s(2 i)}^{v} \ell_{y}^{2}+\sum_{i=1}^{2} k_{s(2 i)}^{h} a_{z} \ell_{z} \\
& K_{4,15}=K_{15,4}=0
\end{aligned}
$$




$$
\begin{aligned}
& K_{5,5}=\sum_{i=1}^{4} k_{s i}^{h} \ell_{x}^{2} \\
& K_{5,6}=K_{6,5}=0 \\
& K_{5,7}=K_{7,5}=\sum_{i=1}^{2} k_{s(2 i-1)}^{h} \ell_{x} \\
& K_{5,8}=K_{8,5}=0 \\
& K_{5,9}=K_{9,5}=\sum_{i=1}^{2} k_{s(2 i-1)}^{h} a_{z} \ell_{x} \\
& K_{5,10}=K_{10,5}=0 \\
& K_{5,11}=K_{11,5}=0 \\
& K_{5,12}=K_{12,5}=-\sum_{i=1}^{2} k_{s(2 i)}^{h} \ell_{x} \\
& K_{5,13}=K_{13,5}=0 \\
& K_{5,14}=-\sum_{i=1}^{2} k_{s(2 i)}^{h} a_{z} \ell_{x} \\
& K_{5,15}=K_{15,5}=0 \\
& K_{6,6}=\sum_{i=1}^{2} k_{s(2 i-1)}^{v}+\sum_{i=1}^{4} k_{t i}^{v} \\
& K_{6,7}=K_{7,6}=0 \\
& K_{6,8}=\sum_{i=1}^{4}(-1)^{i+1} k_{t i}^{v} d_{x} \\
& K_{6,9}=\sum_{i=1}^{2}(-1)^{i+1} k_{s(2 i-1)}^{v} \ell_{y}+\sum_{i=1}^{2} k_{t i}^{v} d_{y}-\sum_{i=3}^{4} k_{t i}^{v} d_{y} \\
& K_{6,10}=K_{10,6}=0 \\
& K_{6,11}=K_{11,6}=0 \\
& K_{6,12}=K_{12,6}=0 \\
& K_{6,13}=K_{13,6}=0 \\
& K_{6,14}=K_{14,6}=0 \\
& K_{6,15}=K_{15,6}=0 \\
& K_{7,7}=\sum_{i=1}^{2} k_{s(2 i-1)}^{h}+\sum_{i=1}^{4} k_{t i}^{h} \\
& K_{7,8}=K_{8,7}=0 \\
& K_{7,9}=\sum_{i=1}^{2} k_{s(2 i-1)}^{h} a_{z}-\sum_{i=1}^{4} k_{t i}^{h} b_{z} \\
& K_{7,10}=\sum_{i=1}^{4}(-1)^{i} k_{t i}^{h} d_{x} \\
& K_{7,11}=K_{11,7}=0 \\
& K_{7,12}=K_{12,7}=0 \\
& K_{7,13}=K_{13,7}=0 \\
& K_{7,14}=K_{14,7}=0 \\
& K_{7,15}=K_{15,7}=0 \\
& K_{8,8}=\sum_{i=1}^{4} k_{t i}^{v} d_{x}^{2} \\
& K_{8,9}=K_{9,8}=\sum_{i=1}^{2}(-1)^{i+1} k_{t i}^{v} d_{x} d_{y}+\sum_{i=3}^{4}(-1)^{i} k_{t i}^{v} d_{x} d_{y} \\
& K_{8,10}=K_{10,8}=0 \\
& K_{8,11}=K_{11,8}=0 \\
& K_{8,12}=K_{12,8}=0 \\
& K_{8,13}=K_{13,8}=0 \\
& K_{8,14}=K_{14,8}=0 \\
& K_{8,15}=K_{15,8}=0
\end{aligned}
$$




$$
\begin{aligned}
& K_{9,9}=\sum_{i=1}^{2} k_{s(2 i-1)}^{v} l_{y}{ }^{2}+\sum_{i=1}^{2} k_{s(2 i-1)}^{h} a_{z}{ }^{2}+\sum_{i=1}^{4} k_{t i}^{v} d_{y}{ }^{2}+\sum_{i=1}^{4} k_{t i}^{h} b_{z}{ }^{2} \quad K_{9,10}=K_{10,9}=\sum_{i=1}^{4}(-1)^{i+1} k_{t i}^{h} d_{x} b_{z} \\
& K_{9,11}=K_{11,9}=0 \quad K_{9,12}=K_{12,9}=0 \\
& k_{9,13}=k_{13,9}=0 \quad K_{9,14}=K_{14,9}=0 \\
& K_{9,15}=K_{15,9}=0 \\
& K_{10,11}=K_{11,10}=0 \\
& K_{10,10}=\sum_{i=1}^{4} k_{t i}^{h} k d_{x}^{2} \\
& K_{10,12}=K_{12,10}=0 \\
& K_{10,13}=K_{13,10}=0 \\
& K_{10,14}=K_{14,10}=0 \\
& K_{10,15}=K_{15,10}=0 \\
& K_{11,11}=\sum_{i=1}^{2} k_{s(2 i)}^{v}+\sum_{i=5}^{8} k_{t i}^{v} \\
& K_{11,12}=K_{12,11}=0 \\
& K_{11,13}=K_{13,11}=\sum_{i=5}^{8}(-1)^{i+1} k_{t i}^{v} d_{x} \\
& K_{1,14}=K_{14,11}=\sum_{i=1}^{2}(-1)^{i+1} k_{s(2 i)}^{v} \ell_{y}+\sum_{i=5}^{6} k_{t i}^{v} d_{y}-\sum_{i=7}^{8} k_{t i}^{v} d_{y} \\
& K_{11,15}=K_{15,11}=0 \\
& K_{12,12}=\sum_{i=1}^{2} k_{s(2 i)}^{h}+\sum_{i=5}^{8} k_{t i}^{h} \\
& K_{12,13}=K_{13,12}=0 \\
& K_{12,14}=K_{14,12}=\sum_{i=1}^{2} k_{s(2 i)}^{h} a_{z}-\sum_{i=5}^{8} k_{t i}^{h} b_{z} \\
& K_{12,15}=K_{15,12}=\sum_{i=5}^{8}(-1)^{i} k_{t i}^{h} d_{x} \\
& K_{13,13}=\sum_{i=5}^{8} k_{t i}^{v} d_{x}^{2} \\
& K_{13,14}=K_{14,13}=\sum_{i=5}^{6}(-1)^{i+1} k_{t i}^{h} d_{x} d_{y}+\sum_{i=7}^{8}(-1)^{i} k_{t i}^{h} d_{x} d_{y} \\
& K_{13,15}=K_{15,13}=0 \\
& K_{14,14}=\sum_{i=1}^{2} k_{s(2 i)}^{v} \ell_{y}{ }^{2}+\sum_{i=1}^{2} k_{s(2 i)}^{h} a_{z}{ }^{2}+\sum_{i=5}^{8} k_{t i}^{v} d_{y}{ }^{2}+\sum_{i=5}^{8} k_{t i}^{h} b_{z}{ }^{2} \\
& K_{14,15}=K_{15,14}=\sum_{i=5}^{8}(-1)^{i+1} k_{t i}^{h} b_{z} d_{x} \\
& K_{15,15}=\sum_{i=5}^{8} k_{t i}^{h} d_{x}{ }^{2}
\end{aligned}
$$

$\{F\}^{T}=\left\{\begin{array}{lllllllllllllll}F_{w_{s}} & F_{v_{s}} & F_{\theta_{s}} & F_{\beta_{s}} & F_{\alpha_{s}} & F_{w_{11}} & F_{v_{11}} & F_{\theta_{11}} & F_{\beta_{11}} & F_{\alpha_{11}} & F_{v_{12}} & F_{v_{12}} & F_{\theta_{12}} & F_{\beta_{12}} & F_{\alpha_{12}}\end{array}\right\}[4.23]$

\section{onde:}

$$
\begin{aligned}
& F_{w_{s}}=0 \\
& F_{v_{s}}=0 \\
& F_{\theta_{s}}=0 \\
& F_{\beta_{s}}=0
\end{aligned}
$$




$$
\begin{aligned}
& F_{\alpha_{s}}=0 \\
& F_{w_{t 1}}=\sum_{i=1}^{4} c_{t i}^{v} \dot{w}_{t r(i)}+\sum_{i=1}^{4} k_{t i}^{v} w_{t r(i)}+\sum_{i=1}^{4} c_{t i}^{v} \dot{w}_{r(i)}+\sum_{i=1}^{4} k_{t i}^{v} w_{r(i)} \\
& F_{v_{t 1}}=\sum_{i=1}^{4} c_{t i}^{h} \dot{v}_{t r(i)}+\sum_{i=1}^{4} k_{t i}^{h} v_{t r(i)} \\
& F_{\theta_{t 1}}=\sum_{i=1}^{4}(-1)^{i+1} c_{t i}^{v} d_{x} \dot{w}_{t r(i)}+\sum_{i=1}^{4}(-1)^{i+1} k_{t i}^{v} d_{x} w_{t r(i)}+\sum_{i=1}^{4}(-1)^{i+1} c_{t i}^{v} d_{x} \dot{w}_{r(i)}+\sum_{i=1}^{4}(-1)^{i+1} k_{t i}^{v} d_{x} w_{r(i)} \\
& F_{\beta_{t 1}}=\sum_{i=1}^{4} c_{t i}^{v} d_{y} \dot{w}_{t r(i)}+\sum_{i=1}^{4} k_{t i}^{v} d_{y} w_{t r(i)}+\sum_{i=1}^{4} c_{t i}^{v} d_{y} \dot{w}_{r(i)}+\sum_{i=1}^{4} k_{t i}^{v} d_{y} w_{r(i)}+\sum_{i=1}^{4} c_{t i}^{h} b_{z} \dot{v}_{t r(i)}+\sum_{i=1}^{4} k_{t i}^{h} b_{z} v_{t r(i)} \\
& F_{\alpha_{t 1}}=\sum_{i=1}^{4}(-1)^{i} c_{t i}^{h} b_{z} \dot{v}_{t r(i)}+\sum_{i=1}^{4}(-1)^{i} k_{t i}^{h} b_{z} v_{t r(i)} \\
& F_{w_{t 2}}=\sum_{i=5}^{8} c_{t i}^{v} \dot{w}_{t r(i)}+\sum_{i=5}^{8} k_{t i}^{v} w_{t r(i)}+\sum_{i=5}^{8} c_{t i}^{v} \dot{w}_{r(i)}+\sum_{i=5}^{8} k_{t i}^{v} w_{r(i)} \\
& F_{v_{t 2}}=\sum_{i=5}^{8} c_{t i}^{h} \dot{v}_{t r(i)}+\sum_{i=5}^{8} k_{t i}^{h} v_{t r(i)} \\
& F_{\theta_{t 2}}=\sum_{i=5}^{8}(-1)^{i+1} c_{t i}^{v} d_{x} \dot{w}_{t r(i)}+\sum_{i=5}^{8}(-1)^{i+1} k_{t i}^{v} d_{x} w_{t r(i)}+\sum_{i=5}^{8}(-1)^{i+1} c_{t i}^{v} d_{x} \dot{w}_{r(i)}+\sum_{i=5}^{8}(-1)^{i+1} k_{t i}^{v} d_{x} w_{r(i)} \\
& F_{\beta_{t 2}}=\sum_{i=5}^{8} c_{t i}^{v} d_{y} \dot{w}_{t r(i)}+\sum_{i=5}^{8} k_{t i}^{v} d_{y} w_{t r(i)}+\sum_{i=5}^{8} c_{t i}^{v} d_{y} \dot{w}_{r(i)}+\sum_{i=5}^{8} k_{t i}^{v} d_{y} w_{r(i)}+\sum_{i=5}^{8} c_{t i}^{h} b_{z} \dot{v}_{t r(i)}+\sum_{i=5}^{8} k_{t i}^{h} b_{z} v_{t r(i)} \\
& F_{\alpha_{t 2}}=\sum_{i=5}^{8}(-1)^{i} c_{t i}^{h} b_{z} \dot{v}_{t r(i)}+\sum_{i=5}^{8}(-1)^{i} k_{t i}^{h} b_{z} v_{t r(i)}
\end{aligned}
$$

sendo:

$$
\begin{aligned}
& w_{r(i)}: \text { irregularidade na roda; } \\
& w_{t r} \text { : irregularidade no trilho no plano vertical; } \\
& v_{t r} \text { : irregularidade no trilho no plano horizontal transversal; }
\end{aligned}
$$

\subsection{MODELO DE ORDEM REDUZIDA DO CARRO}

Para a obtenção do modelo de ordem reduzida do carro, realizou-se uma condensação estática ao seu centro de gravidade, a partir das forças de interação entre as rodas e os trilhos. Assim, o modelo de carregamento ficou reduzido a cinco esforços aplicados no centro de gravidade do carro, a saber: bounce, sway, pitch, roll e yaw. De acordo com a Figura 17, os esforços são determinados da seguinte maneira: 

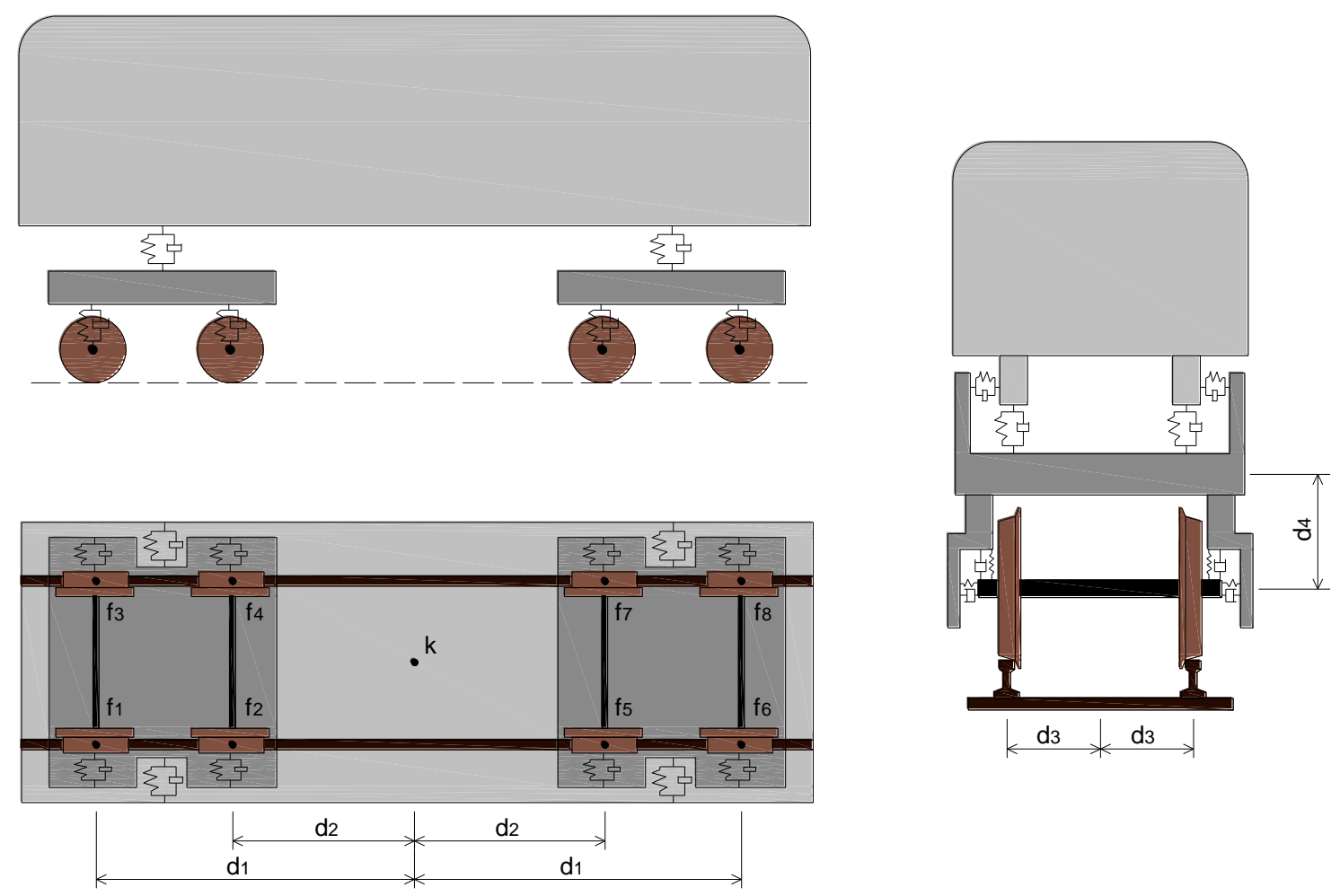

Figura 17: Redução de esforços de interação para o centro de gravidade

$$
\begin{aligned}
& F y_{k}(t)=-\sum_{i=1}^{8} f y_{i}(t) \\
& F z_{k}(t)=-\sum_{i=1}^{8} f z_{i}(t) \\
& M x_{k}(t)=-\left\{\left[f z_{3}(t)+f z_{4}(t)+f z_{7}(t)+f z_{8}(t)\right] \times d_{3}-\left[f z_{1}(t)+f z_{2}(t)+f z_{5}(t)+f z_{6}(t)\right] \times d_{3}\right\} \\
& M y_{k}(t)=-\left\{\left[f z_{1}(t)+f z_{3}(t)-f z_{6}(t)-f z_{8}(t)\right] \times d_{1}+\left[f z_{2}(t)+f z_{4}(t)-f z_{5}(t)-f z_{7}(t)\right] \times d_{2}\right\} \\
& M z_{k}(t)=-\left\{\left[-f y_{1}(t)-f y_{3}(t)+f y_{6}(t)+f y_{8}(t)\right] \times d_{1}+\left[-f y_{2}(t)-f y_{4}(t)+f y_{5}(t)+f y_{7}(t)\right] \times d_{2}\right\}
\end{aligned}
$$

onde:

$F z_{k}(t)$ : é a força na direção $z$ reduzida ao ponto k;

$F y_{k}(t)$ : é a força na direção y reduzida ao ponto k;

$M x_{k}(t)$ : é o momento aplicado em torno do eixo x reduzido ao ponto k;

$M y_{k}(t)$ : é o momento aplicado em torno do eixo y reduzido ao ponto k;

$M z_{k}(t)$ : é o momento aplicado em torno do eixo z reduzido ao ponto k;

$f y_{i}$ : é a força de interação roda-trilho na direção horizontal transversal, definida por: 


$$
\begin{aligned}
& f y_{1}=c_{t 1}^{h}\left(\dot{v}_{t r 1}-\dot{\beta}_{t 1} d_{4}-\dot{\alpha}_{t 1} d_{1}\right)+k_{t 1}^{h}\left(v_{t r 1}-\beta_{t 1} d_{4}-\alpha_{t 1} d_{1}\right) \\
& f y_{2}=c_{t 2}^{h}\left(\dot{v}_{t r 2}-\dot{\beta}_{t 1} d_{4}+\dot{\alpha}_{t 1} d_{2}\right)+k_{t 2}^{h}\left(v_{t r 2}-\beta_{t 1} d_{4}+\alpha_{t 1} d_{2}\right) \\
& f y_{3}=c_{t 3}^{h}\left(\dot{v}_{t r 3}-\dot{\beta}_{t 1} d_{4}-\dot{\alpha}_{t 1} d_{1}\right)+k_{t 3}^{h}\left(v_{t r 3}-\beta_{t 1} d_{4}-\alpha_{t 1} d_{1}\right) \\
& f y_{4}=c_{t 4}^{h}\left(\dot{v}_{t r 4}-\dot{\beta}_{t 1} d_{4}+\dot{\alpha}_{t 1} d_{2}\right)+k_{t 4}^{h}\left(v_{t r 4}-\beta_{t 1} d_{4}+\alpha_{t 1} d_{2}\right) \\
& f y_{5}=c_{t 5}^{h}\left(\dot{v}_{t r 5}-\dot{\beta}_{t 2} d_{4}-\dot{\alpha}_{t 2} d_{2}\right)+k_{t 5}^{h}\left(v_{t r 5}-\beta_{t 2} d_{4}-\alpha_{t 2} d_{2}\right) \\
& f y_{6}=c_{t 6}^{h}\left(\dot{v}_{t r 6}-\dot{\beta}_{t 2} d_{4}+\dot{\alpha}_{t 2} d_{1}\right)+k_{t 6}^{h}\left(v_{t r 6}-\beta_{t 2} d_{4}+\alpha_{t 2} d_{1}\right) \\
& f y_{7}=c_{t 7}^{h}\left(\dot{v}_{t r 7}-\dot{\beta}_{t 2} d_{4}-\dot{\alpha}_{t 2} d_{2}\right)+k_{t 7}^{h}\left(v_{t r 7}-\beta_{t 2} d_{4}-\alpha_{t 2} d_{2}\right) \\
& f y_{8}=c_{t 8}^{h}\left(\dot{v}_{t r 8}-\dot{\beta}_{t 2} d_{4}+\dot{\alpha}_{t 2} d_{1}\right)+k_{t 8}^{h}\left(v_{t r 8}-\beta_{t 2} d_{4}+\alpha_{t 2} d_{1}\right)
\end{aligned}
$$

$f_{z_{k}}$ : é a força de interação roda-trilho na direção vertical, definida por:

$$
\begin{aligned}
& f_{z_{1}}=c_{t 1}^{v}\left(\dot{w}_{t r 1}+\dot{w}_{r 1}+\dot{\theta}_{t 1} d_{1}+\dot{\beta}_{t 1} d_{3}\right)+k_{t 1}^{v}\left(w_{t r 1}+w_{r 1}+\theta_{t 1} d_{1}+\beta_{t 1} d_{3}\right) \\
& f_{z_{2}}=c_{t 2}^{v}\left(\dot{w}_{t r 2}+\dot{w}_{r 2}-\dot{\theta}_{t 1} d_{2}+\dot{\beta}_{t 1} d_{3}\right)+k_{t 2}^{v}\left(w_{t r 2}+w_{r 2}-\theta_{t 1} d_{2}+\beta_{t 1} d_{3}\right) \\
& f_{z_{3}}=c_{t 3}^{v}\left(\dot{w}_{t r 3}+\dot{w}_{r 3}+\dot{\theta}_{t 1} d_{1}-\dot{\beta}_{t 1} d_{3}\right)+k_{t 3}^{v}\left(w_{t r 3}+w_{r 3}+\theta_{t 1} d_{1}-\beta_{t 1} d_{3}\right) \\
& f_{z_{4}}=c_{t 4}^{v}\left(\dot{w}_{t r 4}+\dot{w}_{r 4}-\dot{\theta}_{t 1} d_{2}-\dot{\beta}_{t 1} d_{3}\right)+k_{t 4}^{v}\left(w_{t r 4}+w_{r 4}-\theta_{t 1} d_{2}-\beta_{t 1} d_{3}\right) \\
& f_{z_{5}}=c_{t 5}^{v}\left(\dot{w}_{t r 5}+\dot{w}_{r 5}+\dot{\theta}_{t 2} d_{2}+\dot{\beta}_{t 2} d_{3}\right)+k_{t 5}^{v}\left(w_{t r 5}+w_{r 5}+\theta_{t 2} d_{2}+\beta_{t 2} d_{3}\right) \\
& f_{z_{6}}=c_{t 6}^{v}\left(\dot{w}_{t r 6}+\dot{w}_{r 6}-\dot{\theta}_{t 2} d_{1}+\dot{\beta}_{t 2} d_{3}\right)+k_{t 6}^{v}\left(w_{t r 6}+w_{r 6}-\theta_{t 2} d_{1}+\beta_{t 2} d_{3}\right) \\
& f z_{7}=c_{t 7}^{v}\left(\dot{w}_{t r 7}+\dot{w}_{r 7}+\dot{\theta}_{t 2} d_{2}-\dot{\beta}_{t 2} d_{3}\right)+k_{t 7}^{v}\left(w_{t r 7}+w_{r 7}+\theta_{t 2} d_{2}-\beta_{t 2} d_{3}\right) \\
& f_{z_{8}}=c_{t 8}^{v}\left(\dot{w}_{t r 8}+\dot{w}_{r 8}-\dot{\theta}_{t 2} d_{1}-\dot{\beta}_{t 2} d_{3}\right)+k_{t 8}^{v}\left(w_{t r 8}+w_{r 8}-\theta_{t 2} d_{1}-\beta_{t 2} d_{3}\right)
\end{aligned}
$$

Cabe lembrar que no caso deste estudo de caso, em que se tem uma ponte com duas vias, considerou-se ainda a excentricidade da pista e ainda, a transferência dos esforços ao centro de gravidade da ponte.

\subsection{APLICAÇÃO DOS ESFORÇOS DE CONTATO NO MODELO ESTRUTURAL}

Com os esforços condensados estaticamente no centro de gravidade do carro, partiu-se para a modelagem do trem trafegando sobre a ponte. Esses esforços serão aplicados a um modelo de baixa hierarquia da ponte (estrutura unifilar), dividido em elementos finitos de barras tridimensionais, sendo que em cada nó da ponte discretizada serão especificados os cinco esforços reduzidos. 


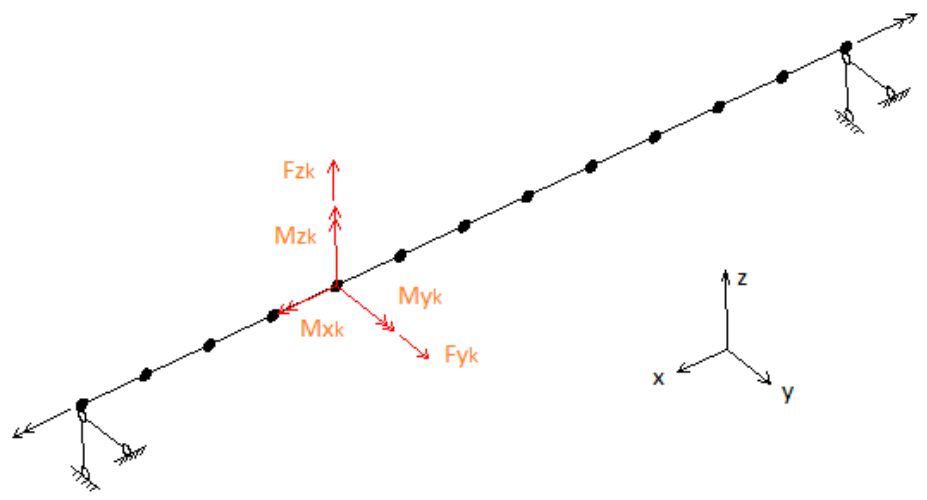

Figura 18: Modelo unifilar da ponte 


\section{ESTUDO DE CASO: FORÇAS NO CONTATO RODA-TRILHO E ESFORÇOS MÓVEIS ESTATICAMENTE REDUZIDOS AO CENTRO DE GRAVIDADE DO CARRO}

\subsection{MODELO DO CARRO}

O carro foi modelado com a utilização do software ADINA - Automatic Dynamic Incremental Nonlinear Analysis, cujos parâmetros foram apresentados na Tabela 3 deste trabalho. $O$ modelo foi construído considerando elementos de barras tridimensionais discretizadas do tipo beam (na terminologia do ADINA), sendo que entre as barras de uma mesma estrutura, vagão ou truque, existem ligações rígidas entre os elementos para garantir o comportamento de corpo rígido. As molas e os amortecedores foram representados por elementos de barra do tipo spring (na terminologia do ADINA), aos quais é possível atribuir propriedades de rigidez e amortecimento. Na Figura 19 é apresentado o modelo veicular.

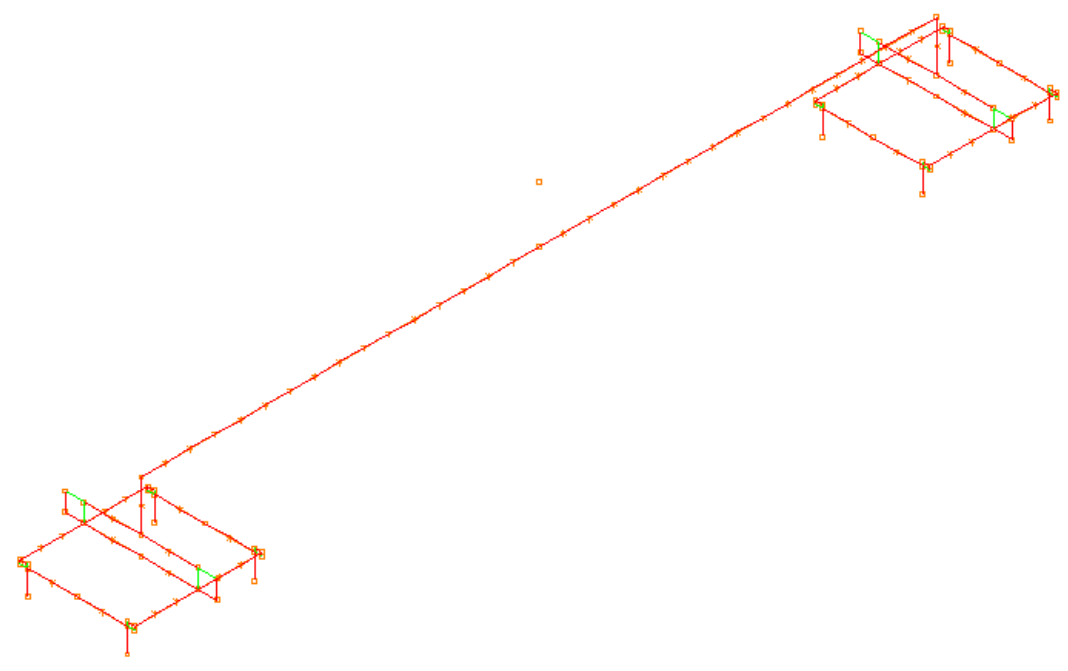

Figura 19: Modelo veicular utilizando o ADINA

Para representar as irregularidades nos trilhos e nas rodas, foram aplicados deslocamentos no contato roda-trilho, regidos pelas funções apresentadas no item 3.2 deste trabalho. As Figuras 20 a 27 apresentam as funções utilizadas para as irregularidades em cada cenário, sendo que as irregularidades no plano vertical representam as imperfeições existentes nos trilhos e nas rodas. 


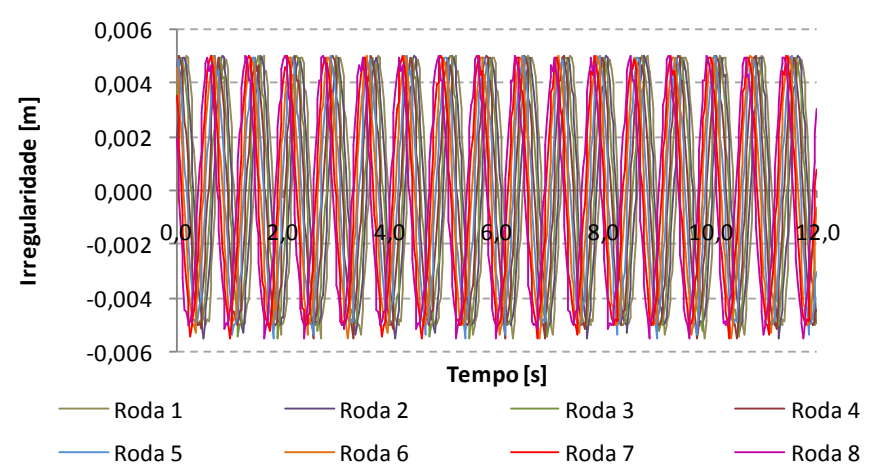

Figura 20: Irregularidades no plano vertical para $n=5$

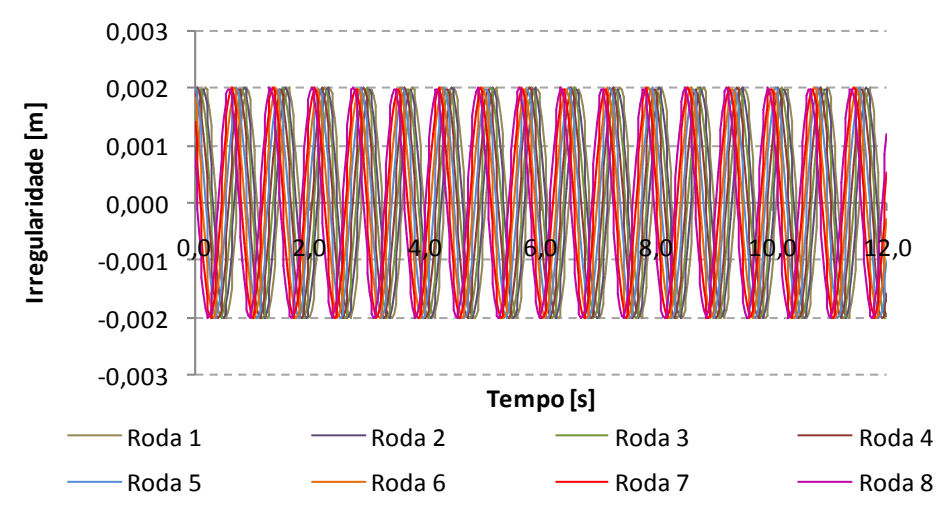

Figura 21: Irregularidades no plano horizontal transversal para $n=5$

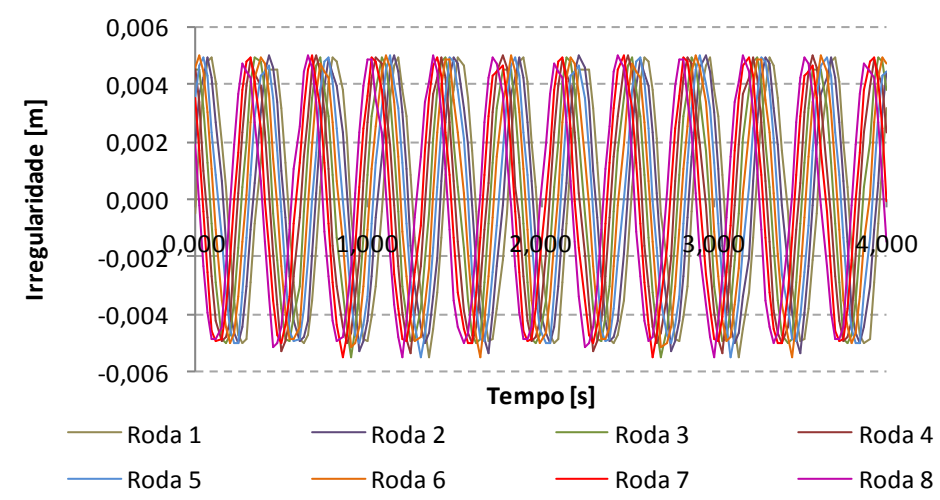

Figura 22: Irregularidades no plano vertical para $n=10$ 


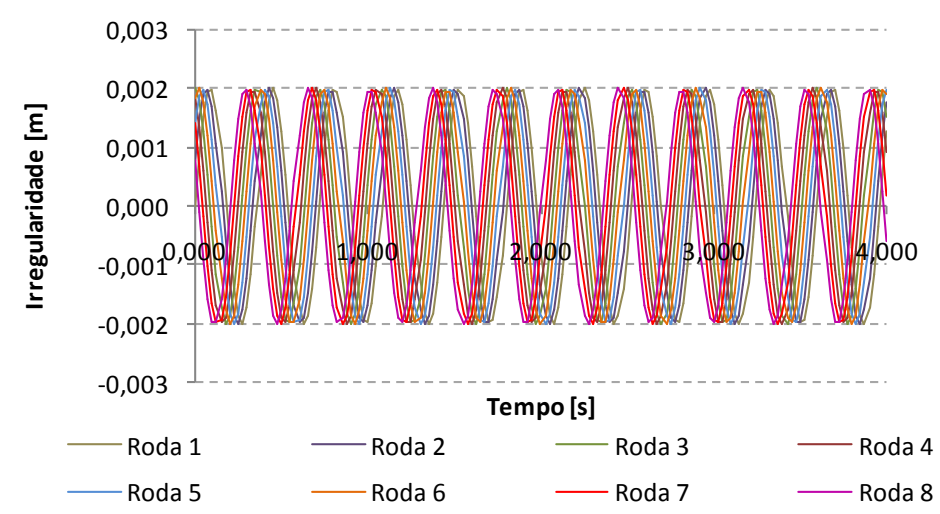

Figura 23: Irregularidades no plano horizontal transversal para $n=10$

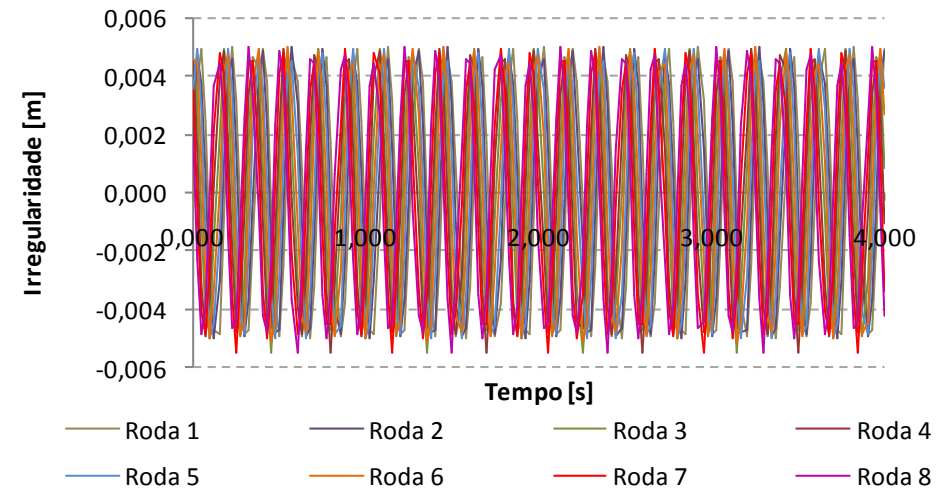

Figura 24: Irregularidades no plano vertical para $n=20$

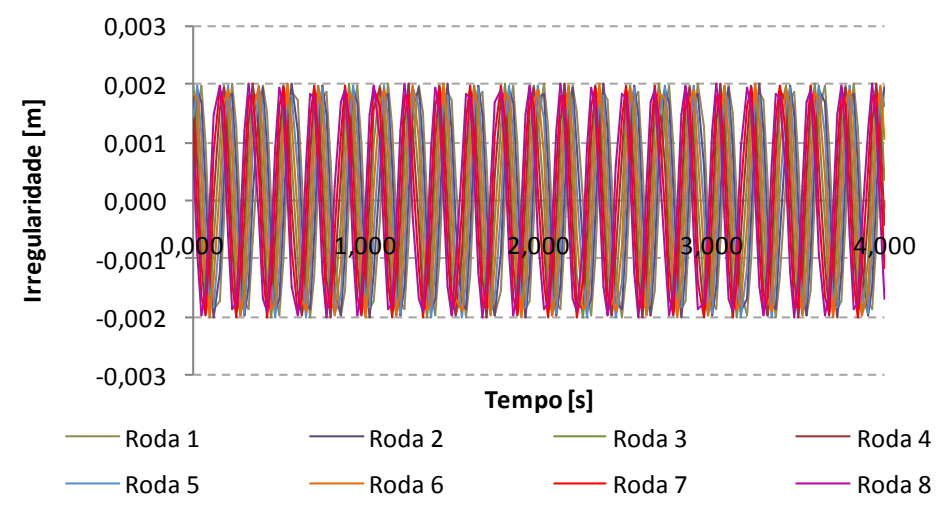

Figura 25: Irregularidades no plano horizontal transversal para $\mathbf{n}=\mathbf{2 0}$ 


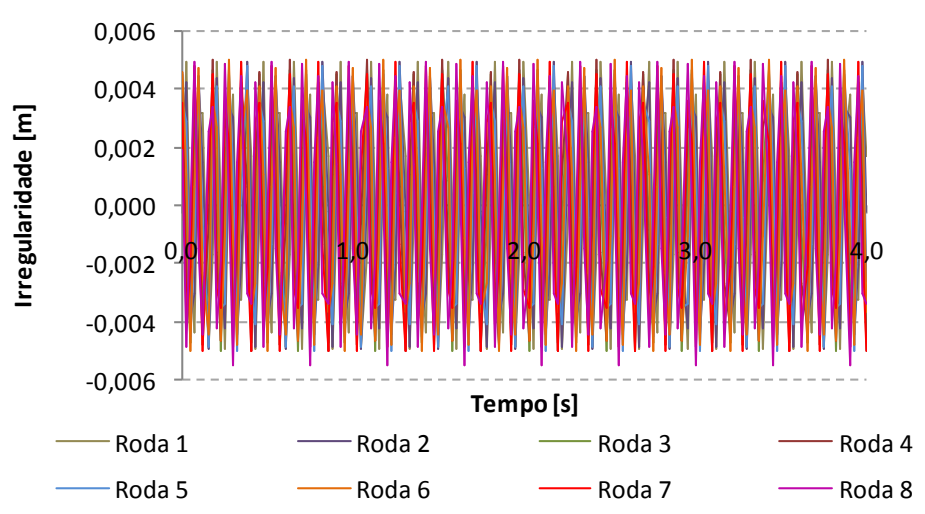

Figura 26: Irregularidades no plano vertical para $n=40$

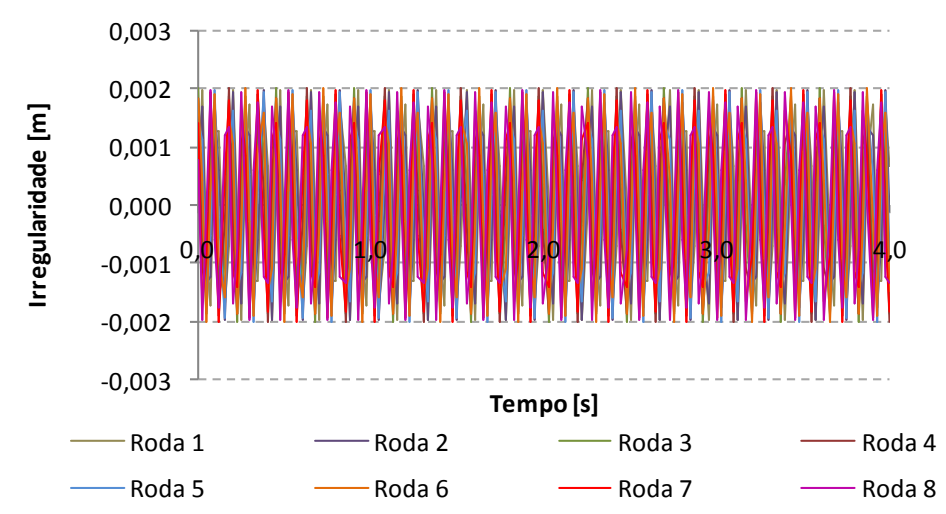

Figura 27: Irregularidades no plano horizontal transversal para $n=40$

As respostas obtidas para o carro com suas respectivas frequências estão apresentadas a seguir.

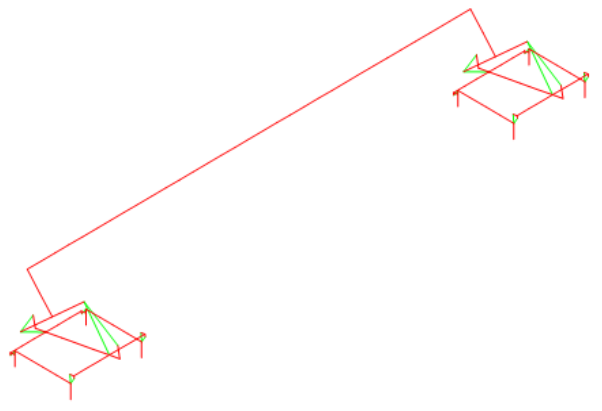

$1^{\circ}$ Modo de vibração - modo de roll

$$
f=0,645 \mathrm{~Hz}
$$

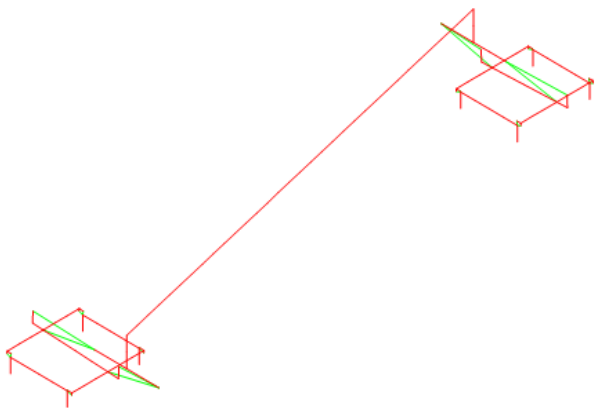

$2^{\circ}$ Modo de vibração - modo de yaw

$$
f=1,215 \mathrm{~Hz}
$$




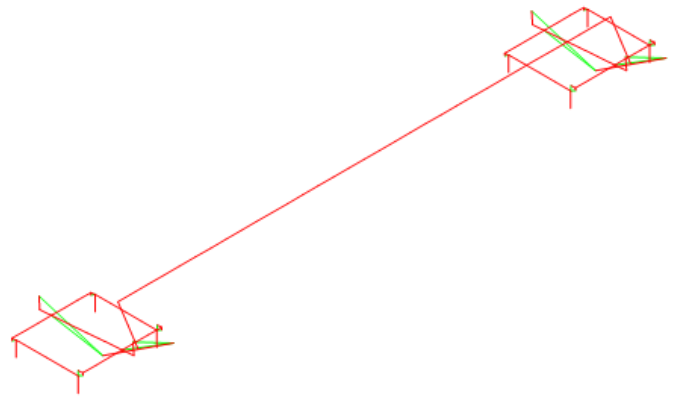

$3^{\circ}$ Modo de vibração - modo de roll e sway $f=1,230 \mathrm{~Hz}$

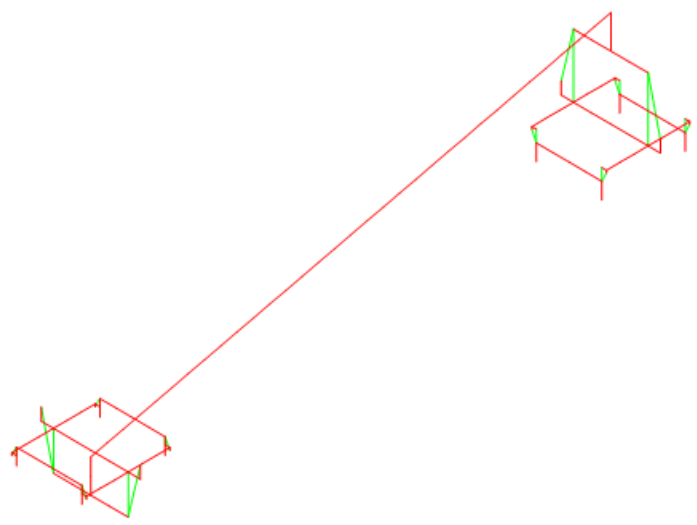

$5^{\circ}$ Modo de vibração - modo de pitch

$$
f=1,677 \mathrm{~Hz}
$$

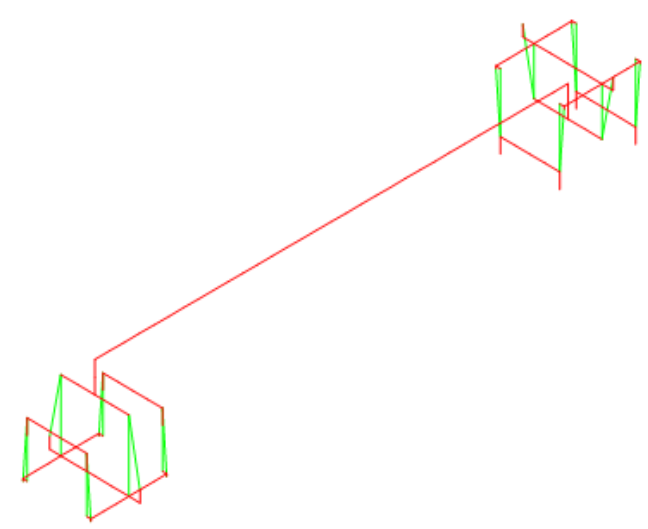

$7^{\circ}$ Modo de vibração - modo de bounce

$$
f=9,055 \mathrm{~Hz}
$$

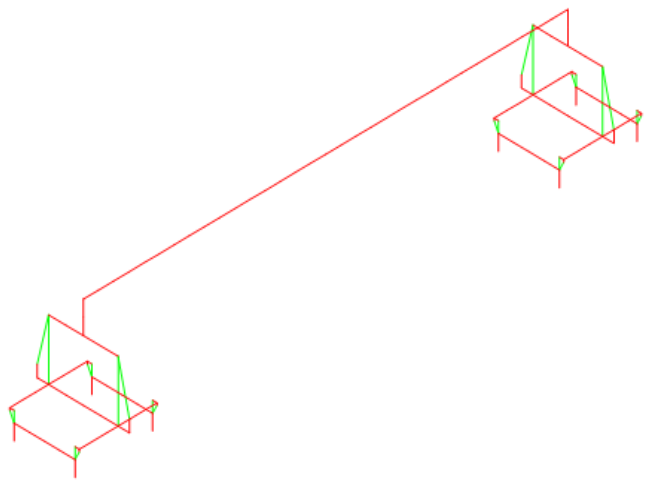

$4^{\circ}$ Modo de vibração - modo de bounce $f=1,334 \mathrm{~Hz}$

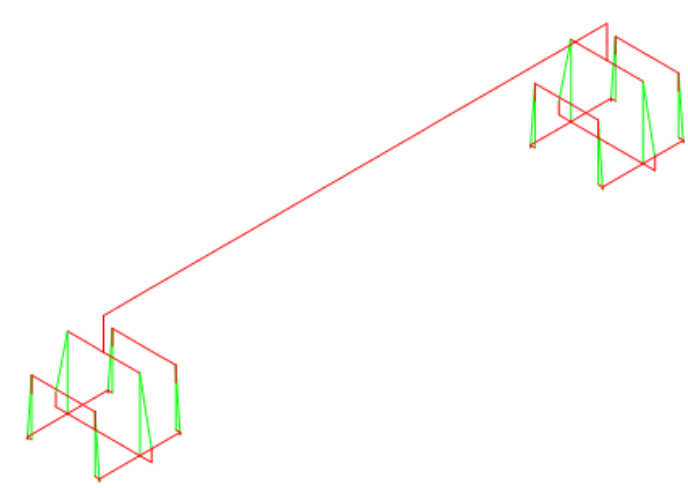

$6^{\circ}$ Modo de vibração - modo de bounce $f=9,044 \mathrm{~Hz}$

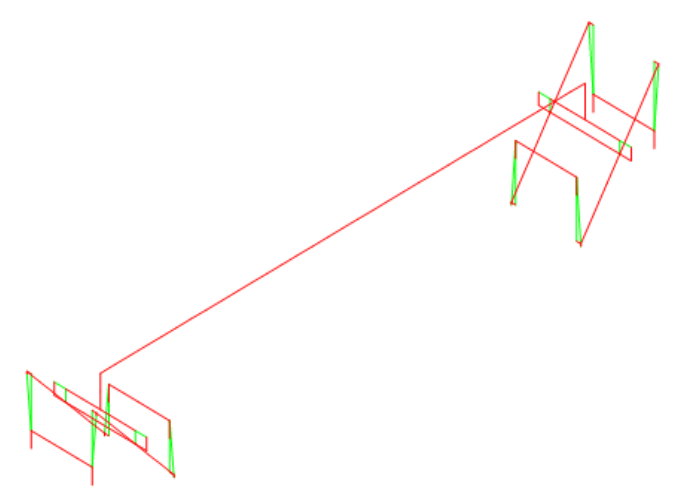

$8^{\circ}$ Modo de vibração - modo de pitch nos truques em sentidos contrários

$$
f=11,720 \mathrm{~Hz}
$$




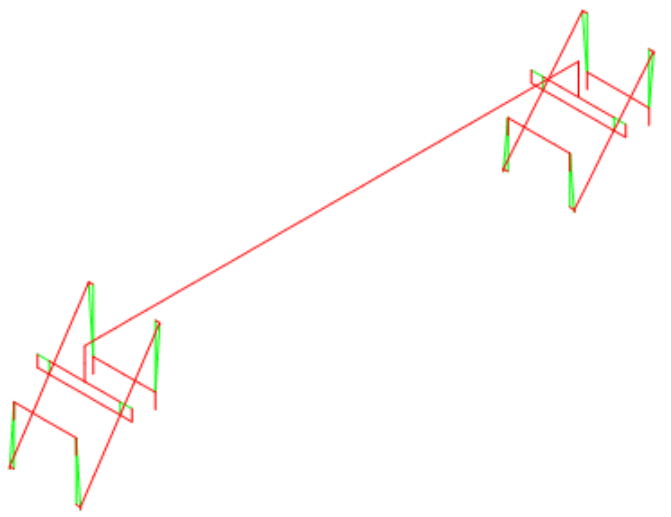

$9^{\circ}$ Modo de vibração - modo de pitch nos truques no mesmo sentido

$$
f=11,720 \mathrm{~Hz}
$$

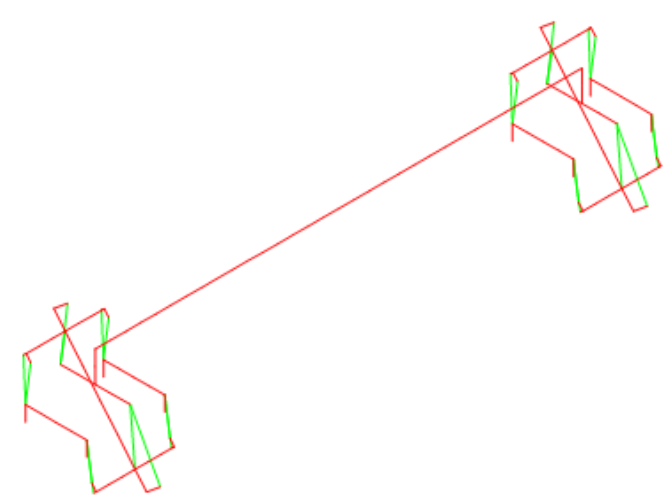

$11^{\circ}$ Modo de vibração - modo de roll nos truques no mesmo sentido

$$
f=15,480 \mathrm{~Hz}
$$

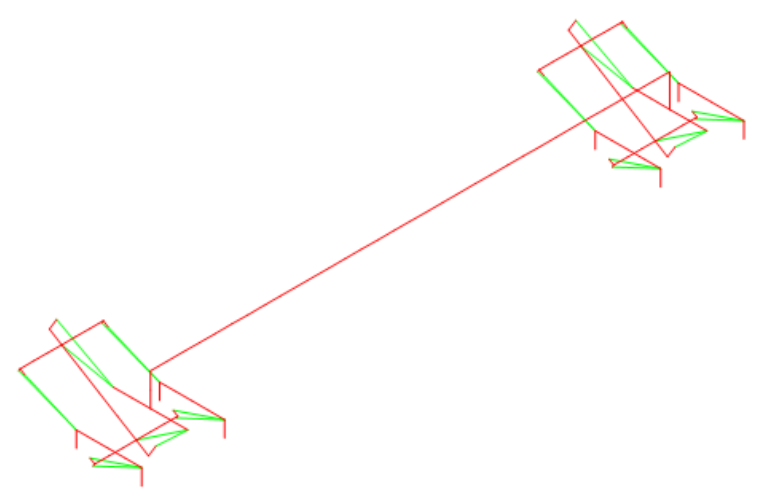

$13^{\circ}$ Modo de vibração - modo de roll e sway nos truques no mesmo sentido

$$
f=21,960 \mathrm{~Hz}
$$

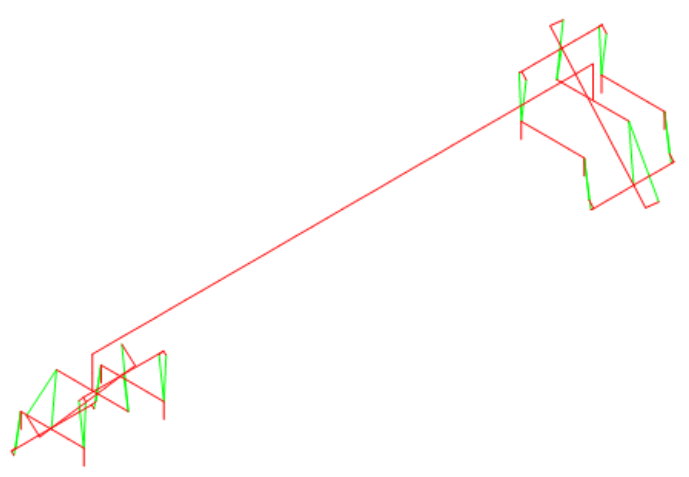

$10^{\circ}$ Modo de vibração - modo de roll nos truques em sentidos contrários

$$
f=15,480 \mathrm{~Hz}
$$

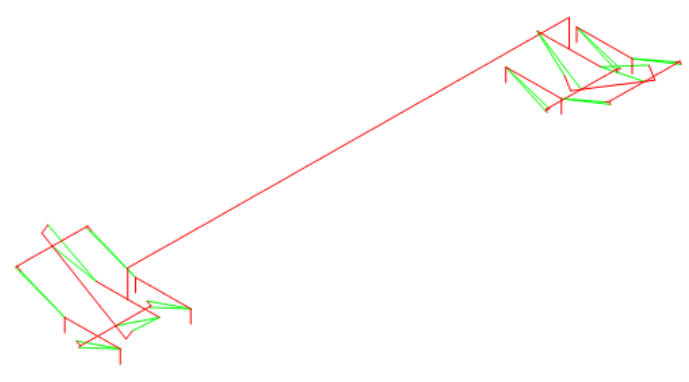

$12^{\circ}$ Modo de vibração - modo de roll e sway nos truques em sentidos contrários $f=21,960 \mathrm{~Hz}$

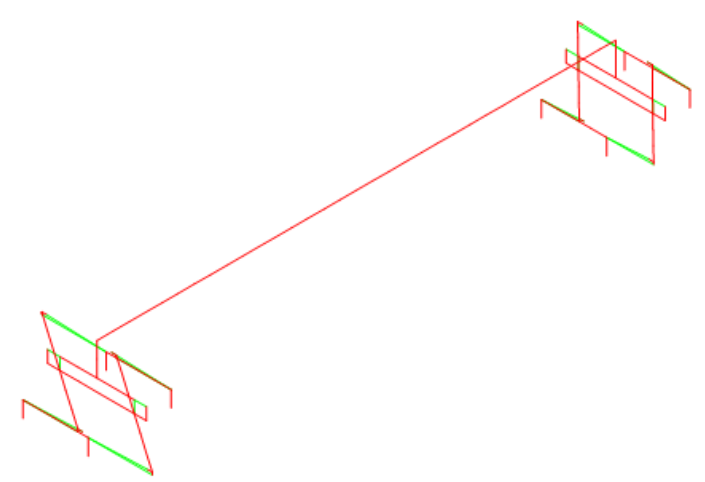

$14^{\circ}$ Modo de vibração - modo de yaw nos truques em sentidos contrários

$$
f=24,990 \mathrm{~Hz}
$$




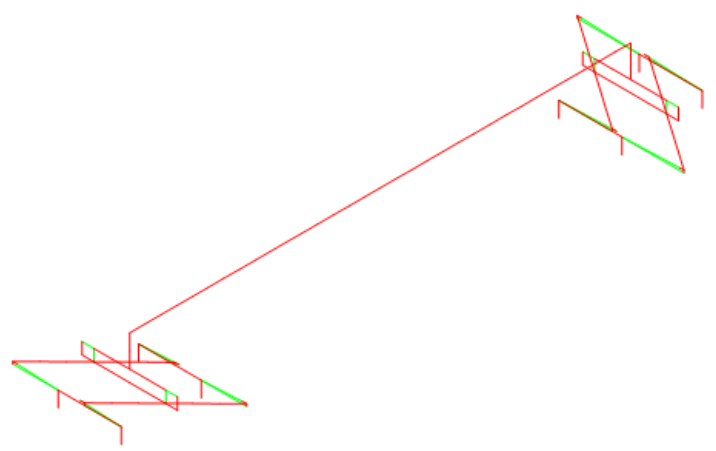

$15^{\circ}$ Modo de vibração - modo de yaw nos

truques em sentidos contrários

$$
f=24,990 \mathrm{~Hz}
$$

Figura 28: Modos de vibração do carro

\subsection{MODELO DA PONTE}

A ponte foi modelada tridimensionalmente no software ADINA, utilizando-se elementos de casca, com apoios em suas extremidades, para a obtenção dos modos de vibração da estrutura (Figura 29). São apresentados os dez primeiros modos da ponte, com suas respectivas frequências de vibração.

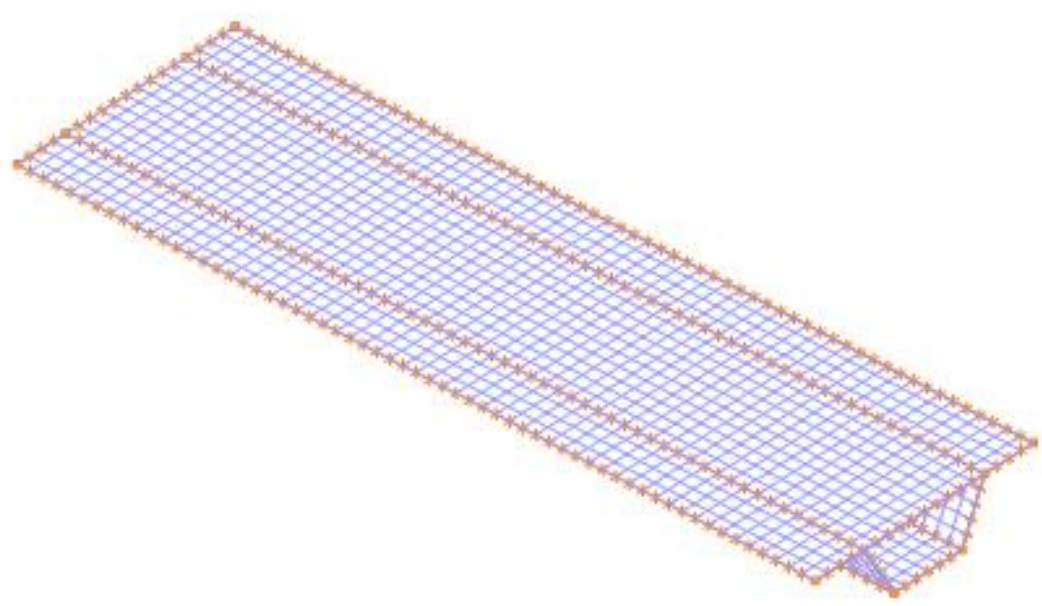

Figura 29: Modelo de casca utilizado para a ponte

A Figura 30 apresenta os dez primeiros modos de vibração da ponte. 


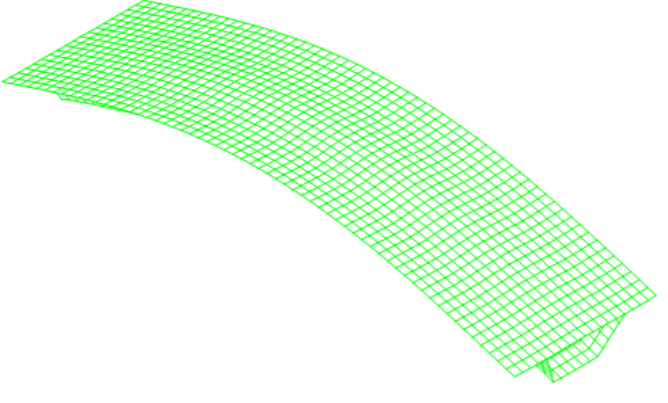

$1^{\circ}$ Modo de vibração - modo de flexão $f=3,307 \mathrm{~Hz}$

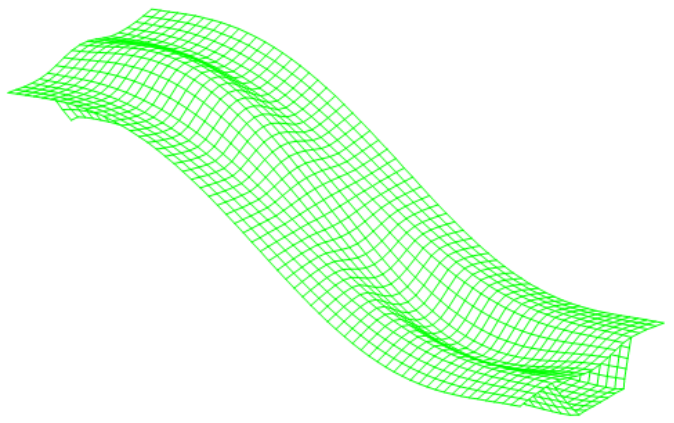

$3^{\circ}$ Modo de vibração - modo de flexão $f=10,560 \mathrm{~Hz}$

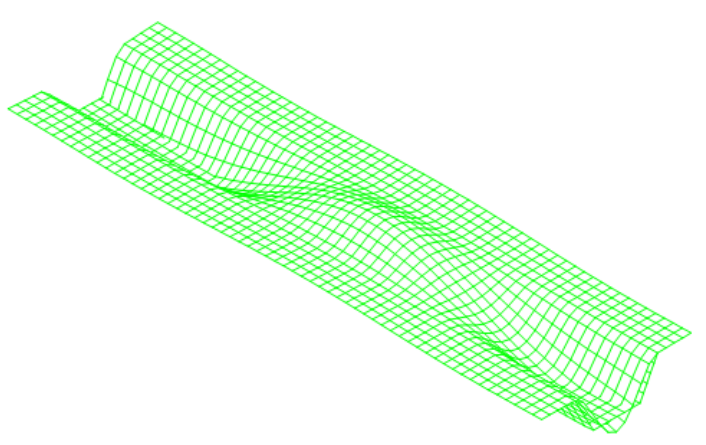

$5^{\circ}$ Modo de vibração - modo de flexão $f=16,30 \mathrm{~Hz}$

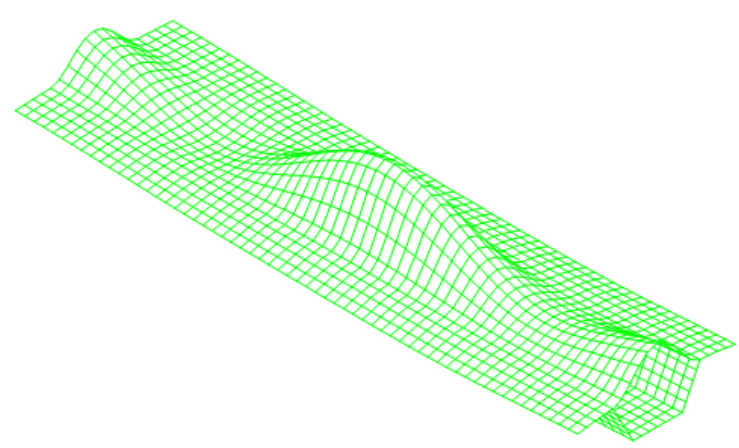

$7^{\circ}$ Modo de vibração - modo de flexão $f=17,410 \mathrm{~Hz}$

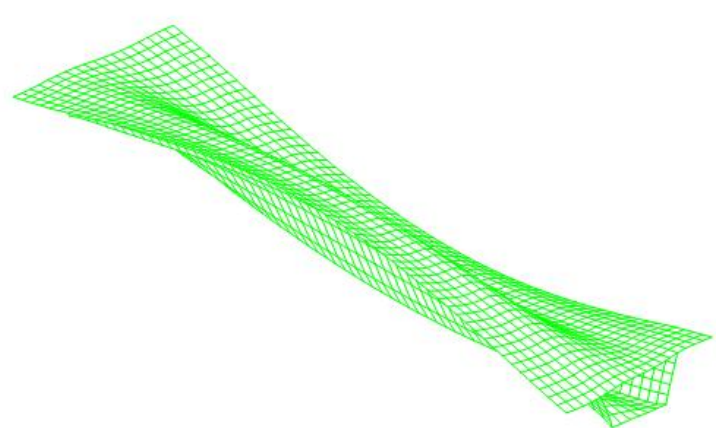

$2^{\circ}$ Modo de vibração - modo de torção $f=10,460 \mathrm{~Hz}$

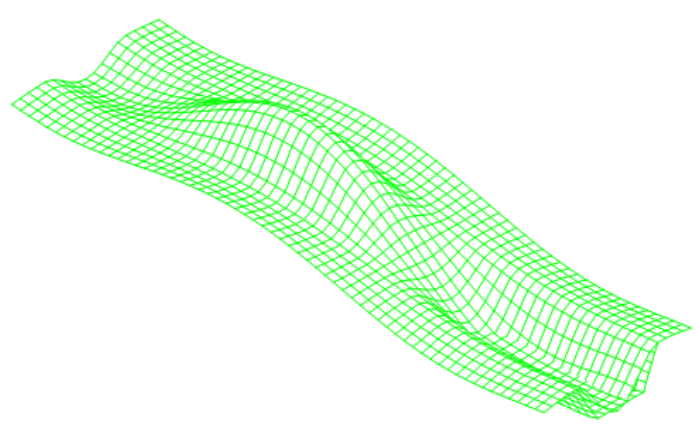

$4^{\circ}$ Modo de vibração - modo de flexão $f=14,970 \mathrm{~Hz}$

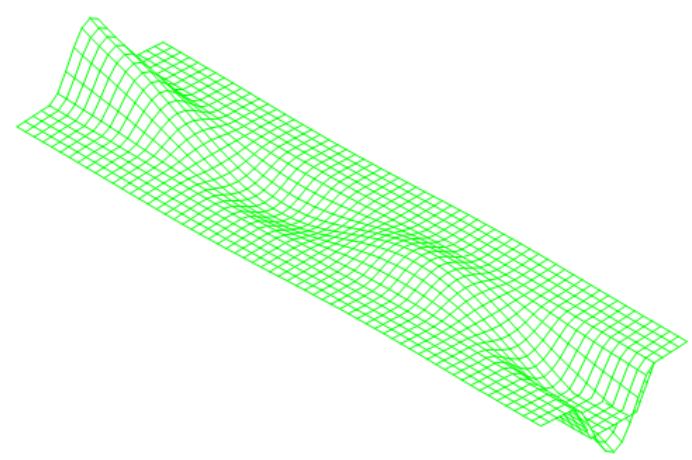

$6^{\circ}$ Modo de vibração - modo de flexão $f=16,730 \mathrm{~Hz}$

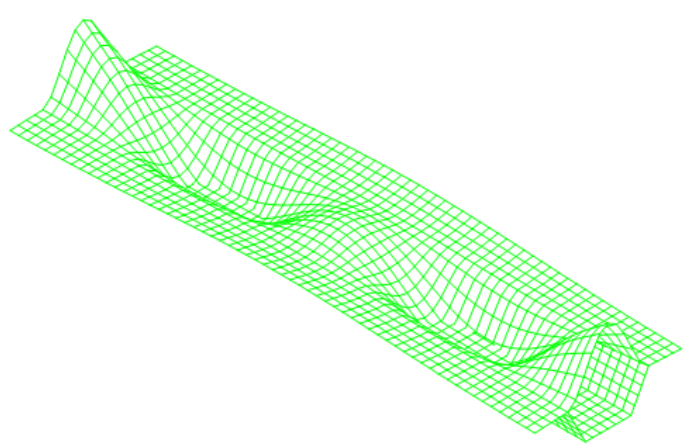

$8^{\circ}$ Modo de vibração - modo de flexão $f=17,670 \mathrm{~Hz}$ 


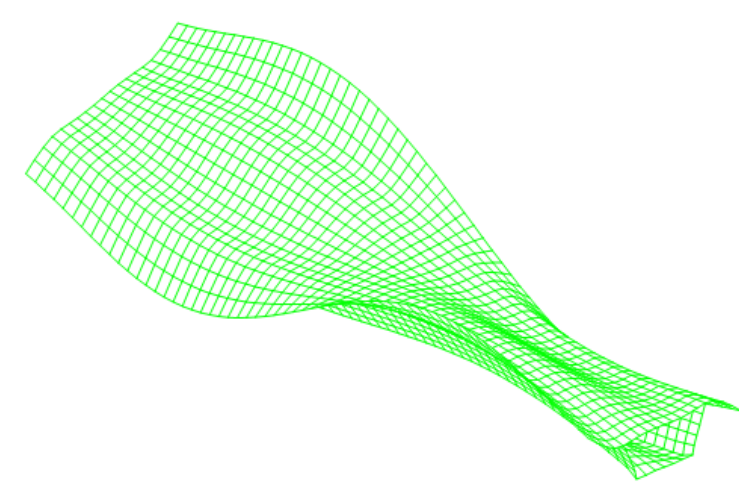

$9^{\circ}$ Modo de vibração - modo de torção $f=18,160 \mathrm{~Hz}$

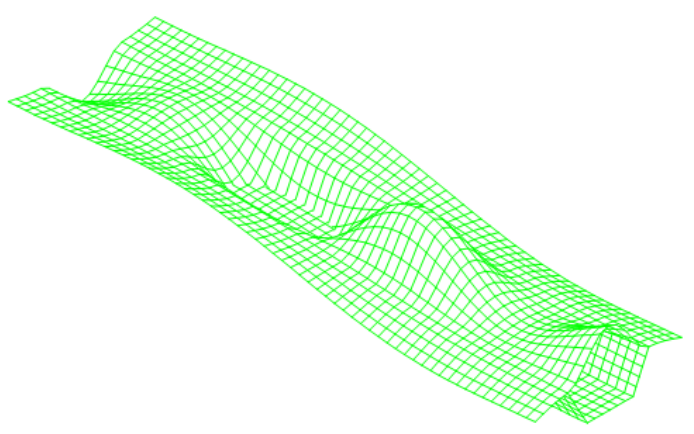

$10^{\circ}$ Modo de vibração - modo de flexão $f=18,480 \mathrm{~Hz}$

Figura 30: Modos de vibração da ponte

\subsection{MODELO UNIFILAR E CARGA A SER APLICADA}

O modelo tridimensional da ponte, com elementos de casca, foi utilizado apenas para a obtenção dos modos de vibração e suas respectivas frequências. Para a modelagem da passagem do trem sobre a ponte, foi considerado um modelo com elementos de barra, conforme definido no item 4.4, cujas propriedades da ponte estão apresentadas na Tabela 4.

Para a obtenção dos esforços de interação roda-trilho reduzidos ao centro de gravidade do carro, considerando a hipótese de tabuleiro rígido indeslocável, foram considerados cinco cenários de análises, variando-se o parâmetro $\mathrm{n}$ apresentado na equação 3.2.

Para os quatro primeiros cenários, foram avaliadas as situações para $\mathrm{n}$ iguais a 5, 10, 20 e 40 admitindo-se uma resposta do carro em regime permanente, ou seja, a presença da irregularidade no trilho e nas rodas existe mesmo antes de o carro entrar na ponte. Cabe ressaltar que os cenários para $n=20$ ou 40 , são situações menos realistas (porque correspondem a trilhos com elevado nível de irregularidade) em combinação com a velocidade $V=20 \mathrm{~m} / \mathrm{s}$, porém, $n=20$ e $V=20 \mathrm{~m} / \mathrm{s}$ pode ser interpretado como sendo equivalente a $n=10$ e $V=40 \mathrm{~m} / \mathrm{s}$, ou ainda $n=5$ e $V=80 \mathrm{~m} / \mathrm{s}$, já se tratando de um trem de alta velocidade. Da mesma forma, $n=40$ e $V=20 \mathrm{~m} / \mathrm{s}$, pode ser interpretado como equivalente a $n=20$ e $V=40 \mathrm{~m} / \mathrm{s}$, ou ainda $n=10$ e $V=80 \mathrm{~m} / \mathrm{s}$, já se tratando de um caso de trem de alta velocidade. 
No quinto cenário analisado, imaginou-se a presença da irregularidade apenas enquanto o trem trafega sobre a ponte, ou seja, os esforços apresentam valores máximos no momento em que o trem entra na ponte, com um regime transiente para os esforços (trecho de 0 a 1,8s). Cabe comentar que este caso foi analisado imaginando-se a existência de ressalto no início da ponte, justificado pela diferença de rigidez da estrutura no instante em que o trem entra na ponte. Neste caso, considerou-se o par $n=10$ e $V=20 \mathrm{~m} / \mathrm{s}$.

A seguir, serão apresentados os esforços para cada cenário analisado. Para o caso com $n=5$, obteve-se a frequência dos esforços de interação igual à 1,33Hz; este valor equivale à frequência do quarto modo de vibração do veículo, ou seja, para este cenário, o carro está ressonante com os esforços de interação.

a) Cenário para $n=5$ :
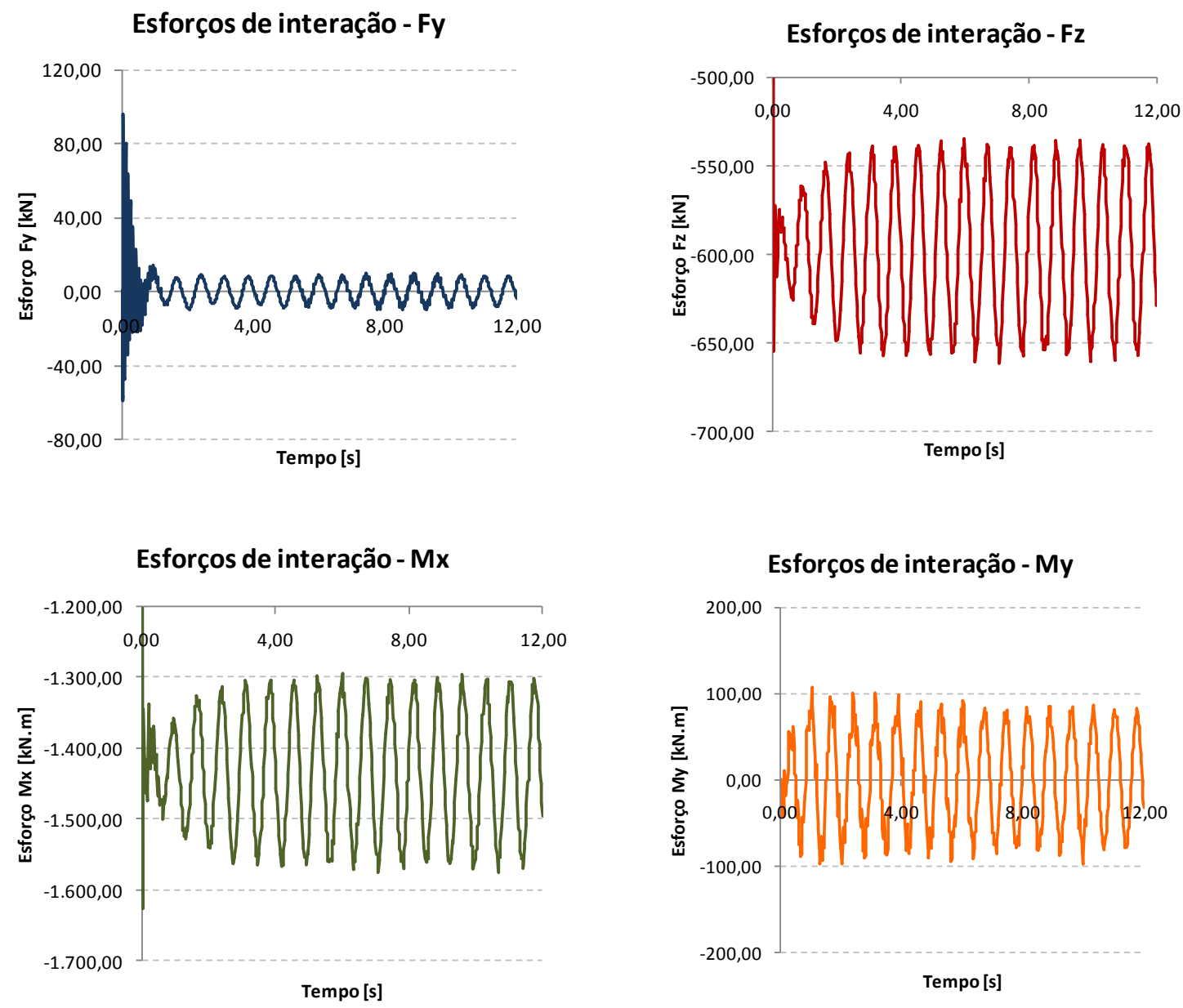


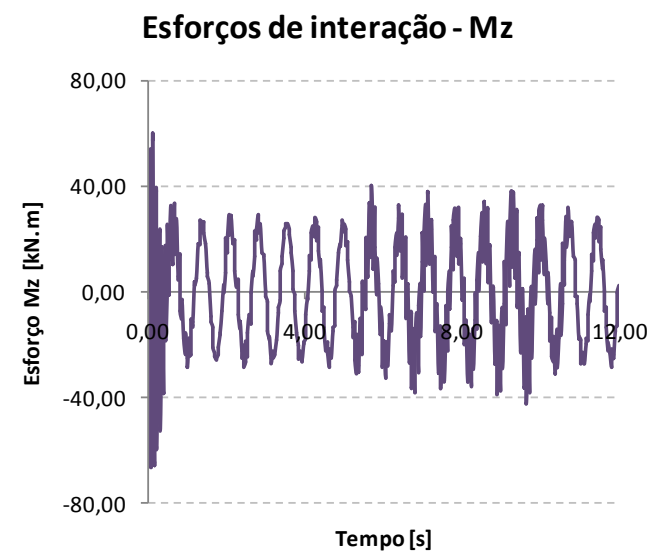

Figura 31: Forças de interação para $n=5$

Os diagramas apresentados na Figura 31

b) Cenário para $n=10$ :

Esforços de interação - Fy

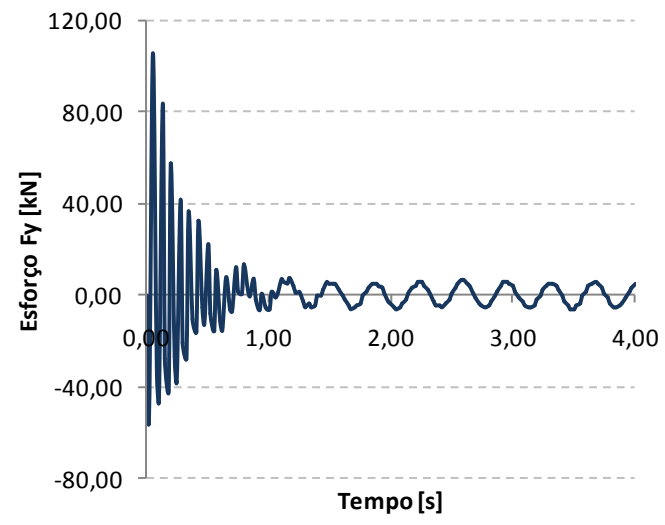

Esforços de interação - Mx

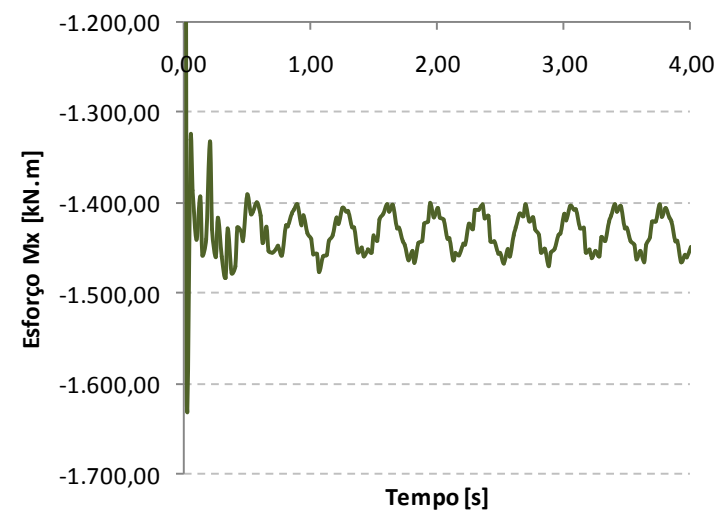

Esforços de interação - Fz

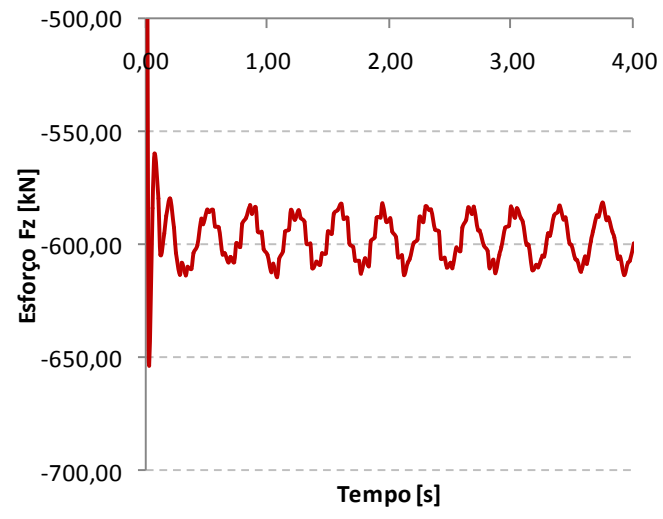

Esforços de interação - My

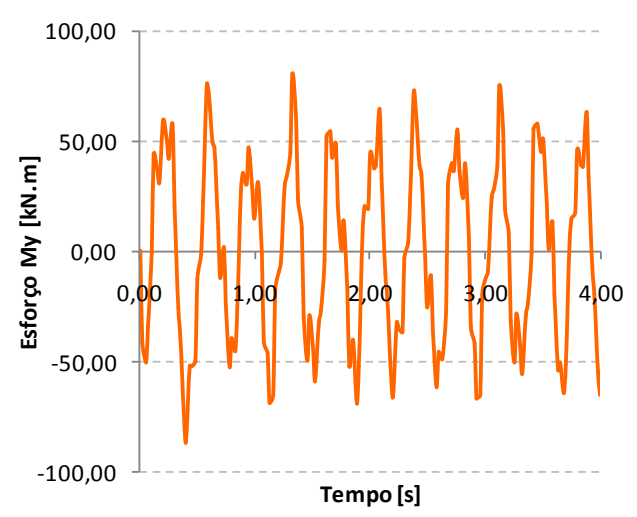




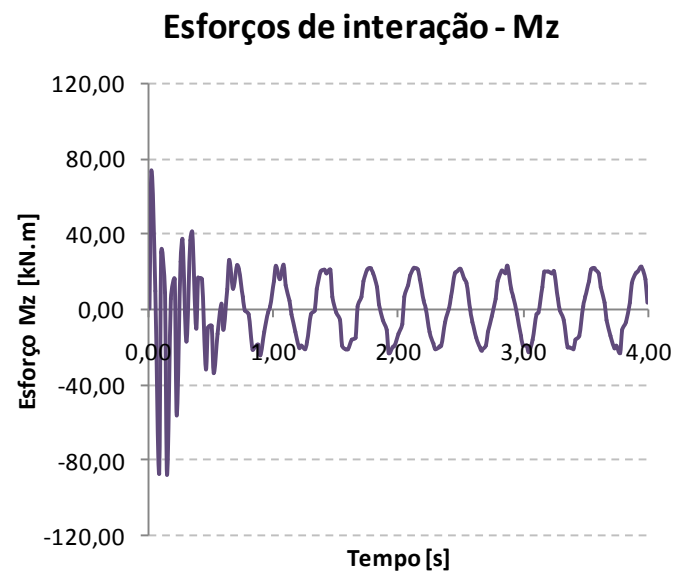

Figura 32: Forças de interação para $n=10$

c) Cenário para $\mathrm{n}=20$ :

Esforços de interação - Fy

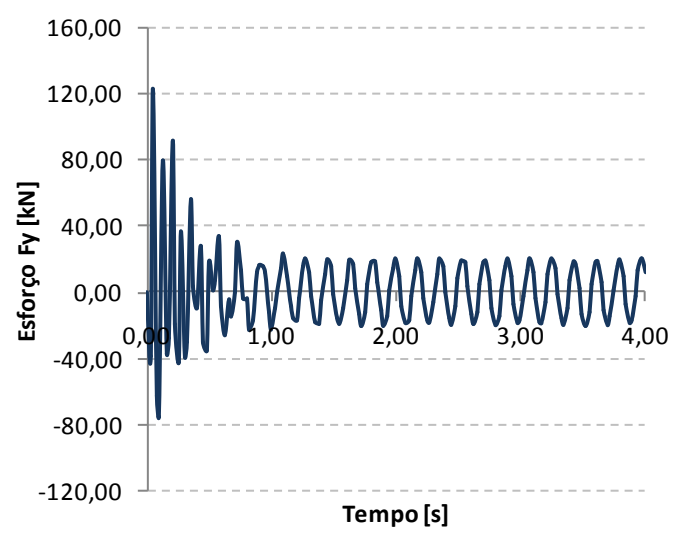

Esforços de interação - Mx

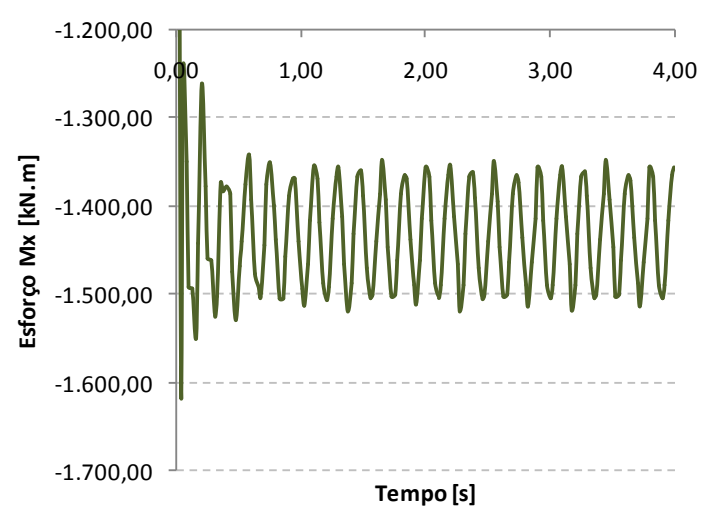

Esforços de interação - Fz
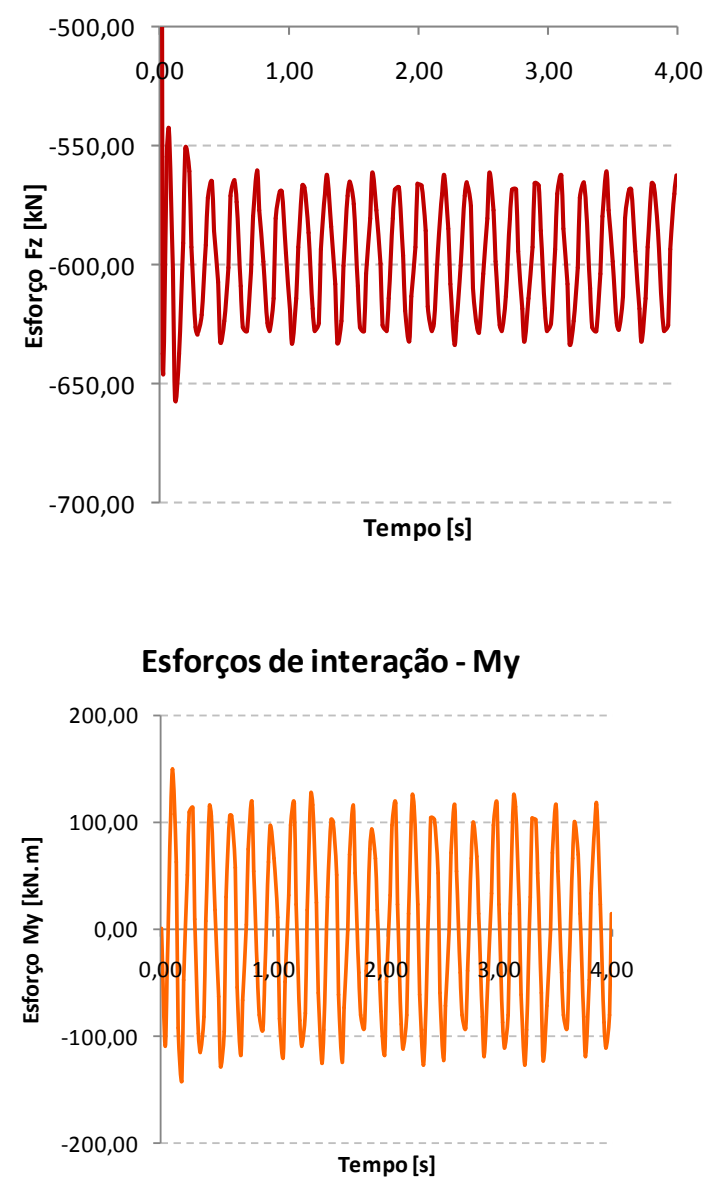


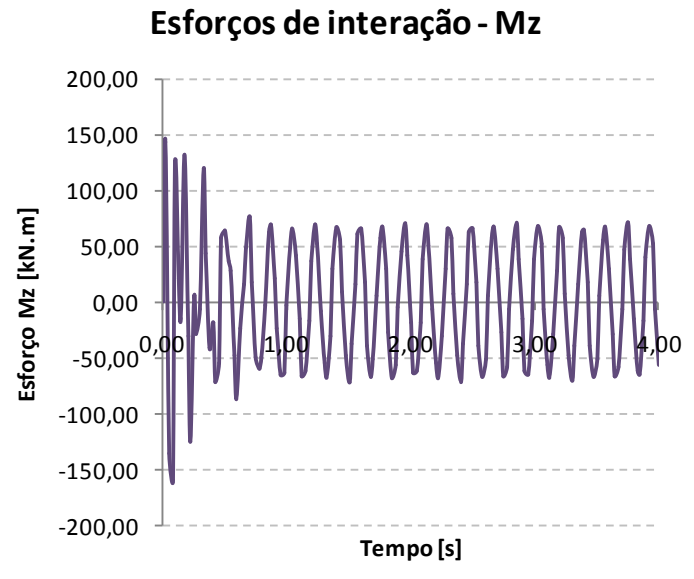

Figura 33: Forças de interação para $n=20$

d) Cenário para $n=40$ :
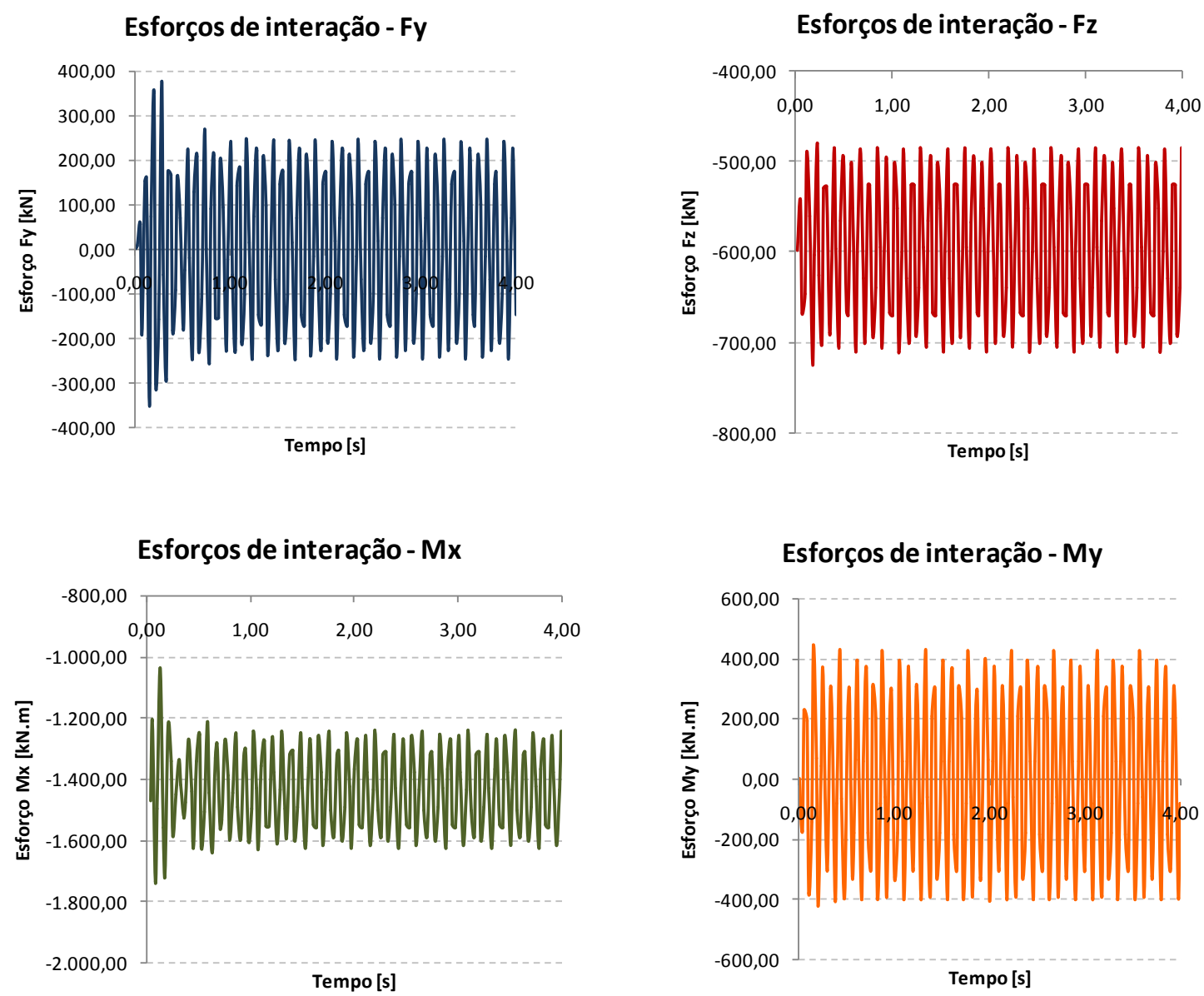


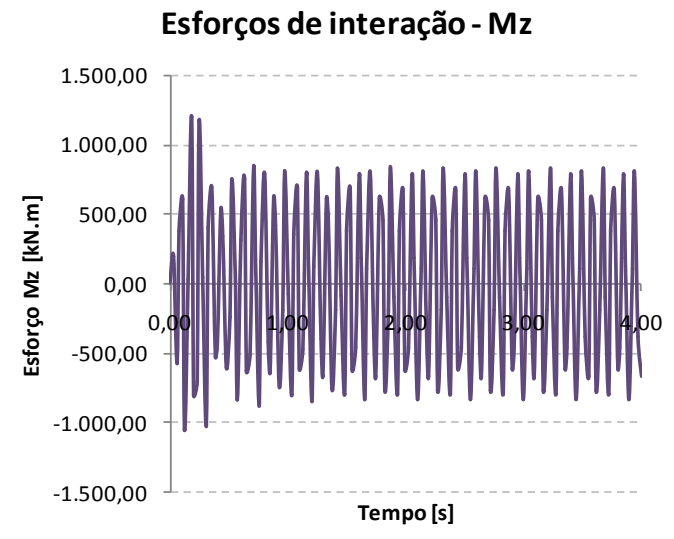

Figura 34: Forças de interação para $n=40$ 


\section{ESTUDO DE CASO: RESPOSTA DINÂMICA ESTRUTURAL EM DECORRÊNCIA DA PASSAGEM DO TREM}

Para aplicação da metodologia apresentada, modelou-se a passagem do trem pela ponte, com peso total de aproximadamente $4.021,0 \mathrm{kN}$, cujos modelos já foram definidos ao longo deste trabalho.

Os resultados decorrentes da passagem do carro modelado com a presença de irregularidades foram comparados com os decorrentes de uma análise quase-estática, aplicando-se os critérios da norma NBR-7187 "Projeto de pontes de concreto armado e de concreto protendido", a fim de se avaliar a influência da presença das irregularidades. Na análise quase-estática, para simular o choque lateral provocado pelas rodas, a norma considera uma força horizontal móvel aplicada no topo do trilho, normal ao eixo da linha, com valor característico igual a $20 \%$ da carga do eixo mais pesado; para cargas verticais, a norma especifica um coeficiente de impacto que é função do comprimento do vão da ponte, que para a ponte considerada neste estudo de caso (36,0m de vão) equivale a 1,321.

A Figura 35 apresenta os resultados obtidos para a análise quase-estática. Nestas análises, consideraram-se todas as situações de carregamento possíveis de ocorrer, ou seja, quais posições o trem poderia ocupar e o número de carros sobre a ponte. As respostas de momento fletor nos planos horizontal e vertical e deslocamento vertical foram avaliadas para a seção do meio do vão da ponte, enquanto que para o momento torçor, analisou-se a seção de um quarto do vão. Cabe comentar que os valores máximos de momentos fletores foram encontrados para a situação em que o centro de gravidade de um carro encontra-se no meio do vão; para o momento torçor, o máximo obtido foi para a situação com dois carros trafegando sobre a ponte, estando o centro de gravidade do primeiro a uma distância de 31,5m do início da ponte e o do segundo a 9,5m; para o deslocamento vertical, a situação crítica também ocorreu com dois carros sobre a ponte, sendo que o centro de gravidade do primeiro encontra-se a 29,5m do início da ponte e o do segundo a 7,0m; no caso das reações nos apoios, os máximos acontecem para dois carros sobre a ponte, estando um deles sobre um dos apoios. 

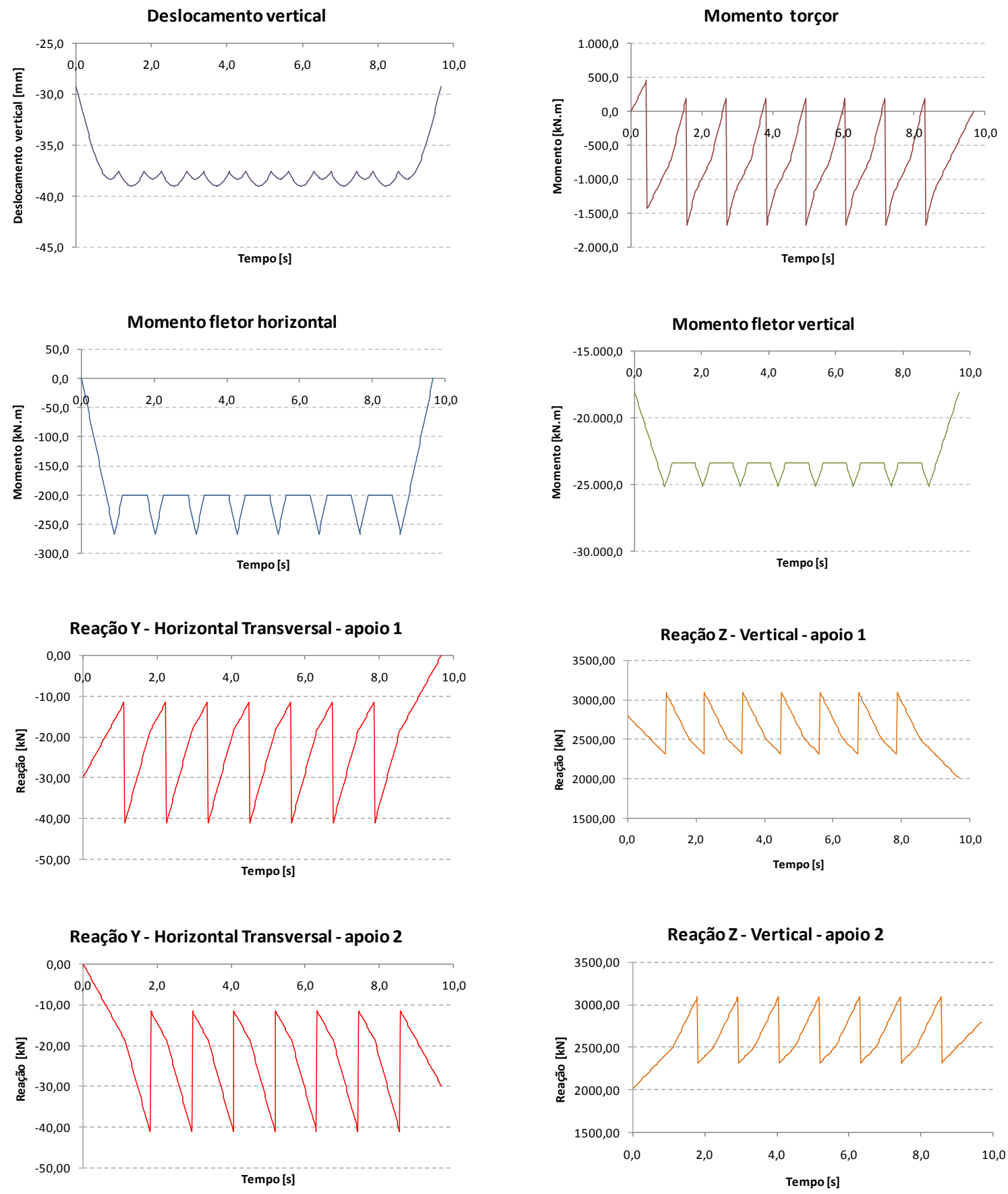

Figura 35: Resultados - análise quase-estática

Como se pode perceber na Figura 35, existe um patamar nos diagramas de momentos fletores nos planos vertical e horizontal. Isto se justifica pela propriedade da linha de influência para este esforço avaliado no meio do vão da ponte. Quando o primeiro carro encontra-se sobre a ponte, o momento fletor máximo ocorre quando o mesmo se encontra no meio do vão da ponte, com valor equivalente à $P \ell / 4$, ou seja, $9 P$ para este estudo de caso, cujo comprimento do vão da ponte equivale à $36,0 \mathrm{~m}$ (Figura 36). 

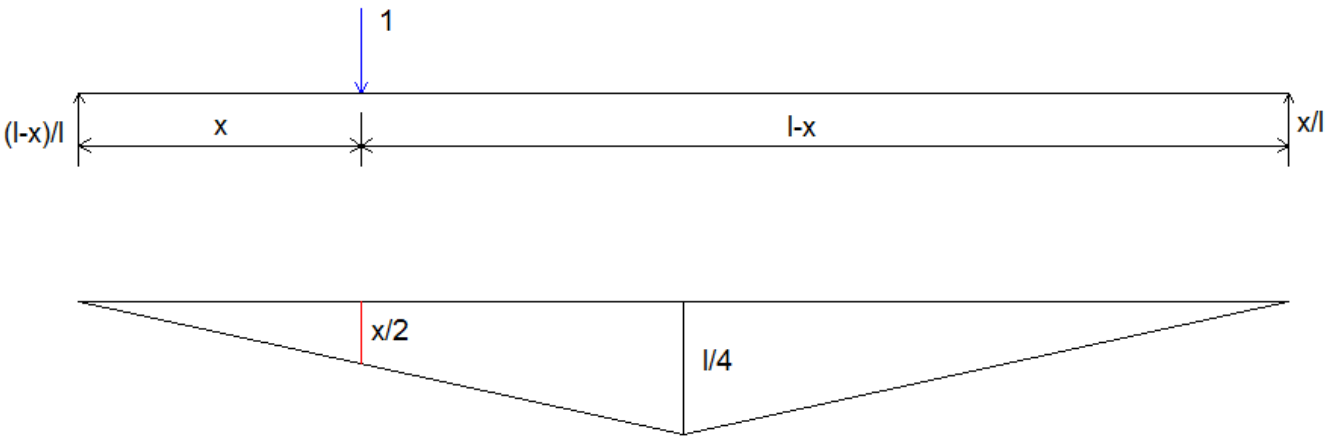

Figura 36: Linha de influência de momento fletor

No instante em que um segundo carro entra na ponte, o momento fletor no meio do vão passa a valer $6,75 P$, permanecendo constante até que o primeiro carro saia da ponte, situação em que este esforço volta a crescer até $9 P$ (Figuras 37 e 38).
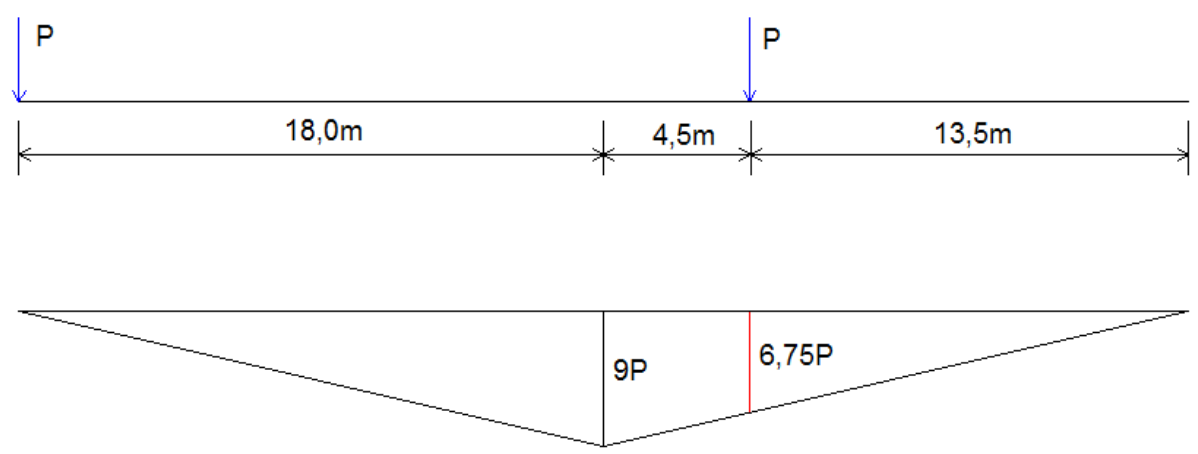

Figura 37: Situação em que um segundo carro entra na ponte
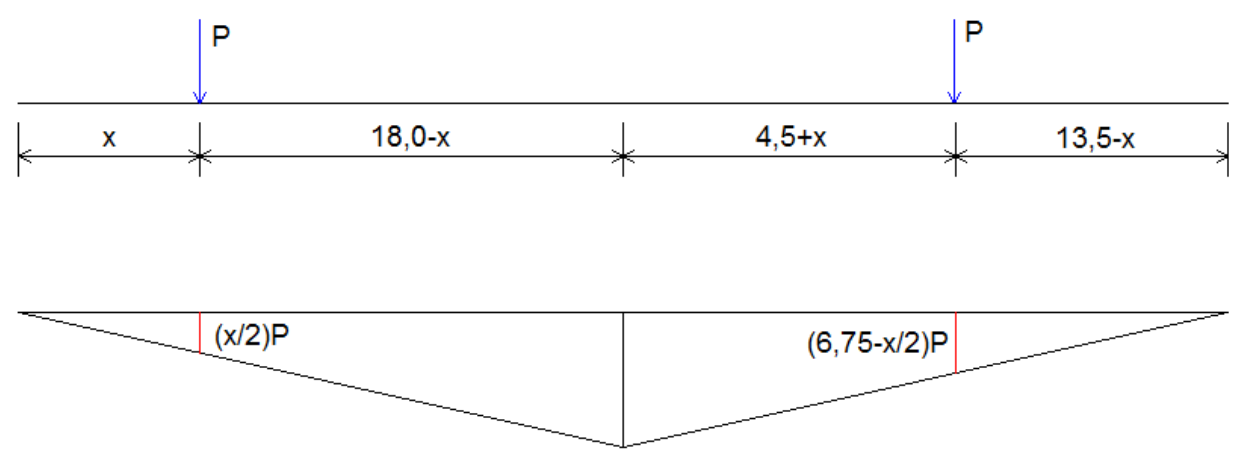

Figura 38: Situação com dois carros sobre a ponte

A Figura 39 ilustra os instantes em que o carro entra e sai da ponte e também, o instante em que o mesmo encontra-se no meio do vão para o diagrama de momento fletor. 


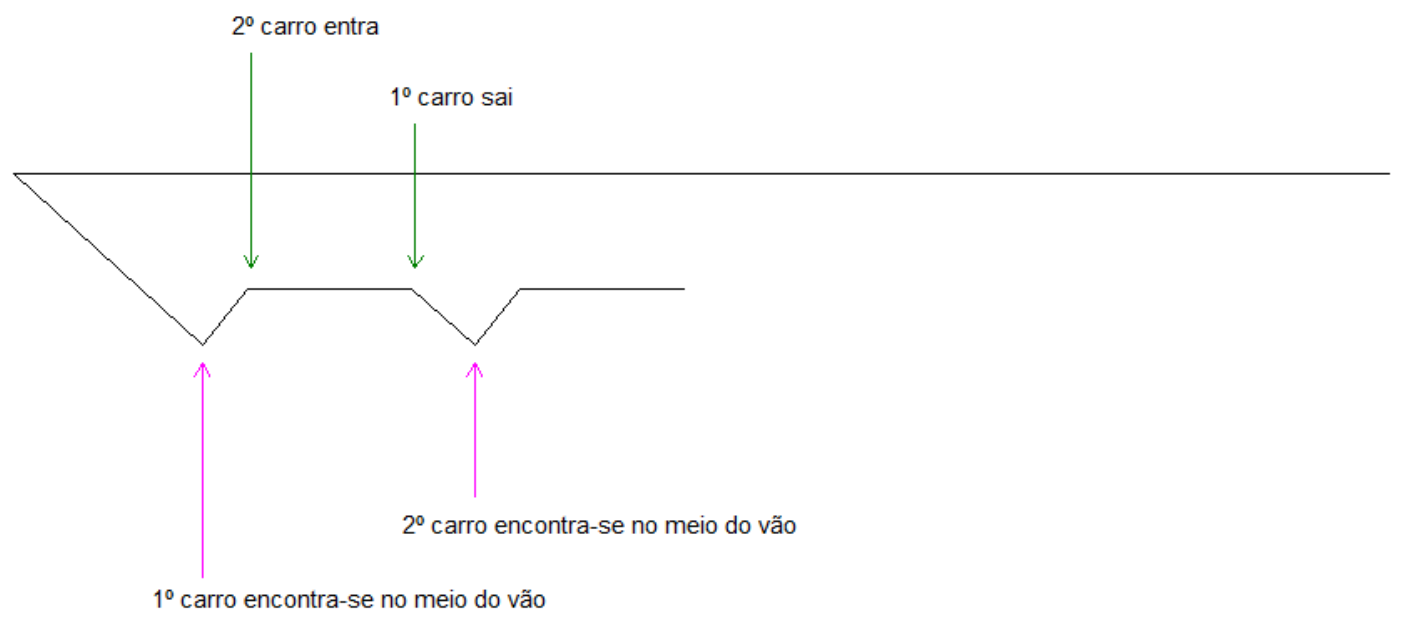

Figura 39: Detalhes dos digramas de momentos fletores

Cabe comentar que, para esta metodologia apresentada, existe um erro ao assumir que o trem entra na ponte no instante em que seu centro de gravidade passa pelo início da mesma. Porém, para efeito dos esforços de momentos e deslocamentos, esta simplificação não interfere nos resultados máximos obtidos, pois eles ocorrem em instantes em que o trem encontra-se no meio do vão. Já para os resultados de reações nos apoios, existe uma diferença, pois quando o centro de gravidade encontra-se sobre o primeiro apoio da ponte e então todo o seu peso está sendo considerado, tem-se uma reação máxima naquele apoio; na realidade, quando o centro de gravidade do trem está nesta posição, apenas metade do trem está sobre a ponte e com isso, a reação no apoio equivale à metade do valor. Quando o trem entra totalmente sobre a ponte, seu centro de gravidade já não está mais no apoio da ponte, e sim, avançado metade do comprimento do carro. É claro que este erro deixa de ter significado em casos de trens de alta velocidade e também, para pontes com vãos muito grandes. Para ilustrar esta redução, apresenta-se o diagrama de reação vertical no primeiro apoio da ponte, conforme Figura 40; como se pode observar, o valor máximo encontrado passou de $3.095 \mathrm{kN}$ para $2.706 \mathrm{kN}$. 


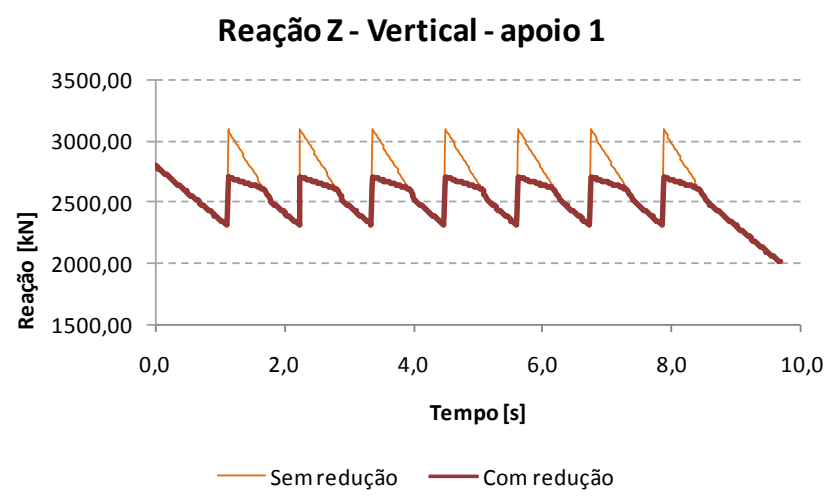

Figura 40: Redução na reação vertical máxima

Para a modelação da análise dinâmica, a simulação da passagem do trem sobre a ponte, ou ainda, segundo esta metodologia, a passagem de cinco esforços de interação reduzidos ao centro de gravidade do carro, foi representada por uma função do tempo $f(t)$ dada por pontos. Neste estudo de caso, foi considerada uma ponte de $L=36 \mathrm{~m}$ de comprimento, discretizada em 72 elementos iguais de comprimento $\Delta x=\frac{L}{72}=0,5 \mathrm{~m}$, num total de 73 nós. Desta forma, sejam: $V=20 \mathrm{~m} / \mathrm{s}$ a velocidade do carro, $\Delta t=0,025 \mathrm{~s}$ o passo de integração no tempo e $\Delta L=22,5 \mathrm{~m}$ o comprimento do carro. Seja ainda a função delta de Kronecker $\delta_{i l}$ para o nó $i=1,2 \ldots 73$ e instante $l \Delta t=0,025 l$ tal que valha zero para $i \neq l$, ou um para $i=l$.

O correspondente carregamento nodal a ser aplicado no nó $i=1,2 \ldots 73$, no instante $j \Delta t$, com $j=1,2 \ldots 720$ será:

$P_{i}(j \Delta t)=f\left[t_{0}+\frac{\Delta x}{V}(i-1)\right] \delta_{i l}$

onde:

$l=j-\frac{\Delta L}{V \Delta t}(k-1)=j-45(k-1)$, sendo $k=1,2, \ldots 8$ o carro do comboio.

A Figura 41 ilustra o esquema de carregamento descrito através da equação 6.1. Como se pode perceber, a ponte foi discretizada em 73 nós. O carregamento é dado inicialmente por apenas um carro, enquanto o primeiro entra na ponte ou o último sai da mesma e, ainda, enquanto se encontra entre as distâncias de $14,5 \mathrm{~m}$ a $22,5 \mathrm{~m}$ do início da ponte, correspondendo esta última situação ao momento em que um segundo carro começa a trafegar sobre ela; o carregamento de dois carros ao mesmo 
tempo, perdurará enquanto um deles já tiver ultrapassado a distância de $22,5 \mathrm{~m}$ do início da ponte, até que saia da mesma.

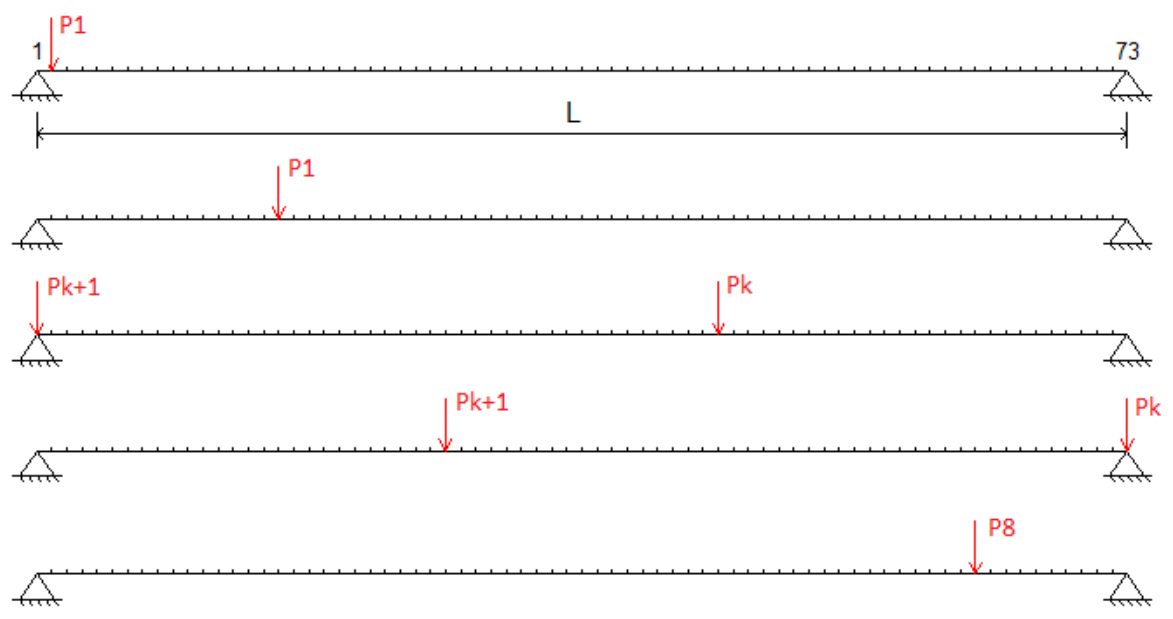

Figura 41: Esquema de carregamento utilizado na análise dinâmica

A seguir, são apresentados os resultados obtidos para as análises dinâmicas descritas para os quatro cenários com esforços de interação em regime permanente. Cabe observar que, assim como comentado nos resultados obtidos para a análise quase-estática, existe um erro nos diagramas obtidos para os esforços de reações no apoio devido ao método simplificado utilizado. Porém, a título de comparação e exemplificação desta metodologia, foram apresentados os diagramas resultantes sem a redução de $50 \%$, conforme já discutido anteriormente. 

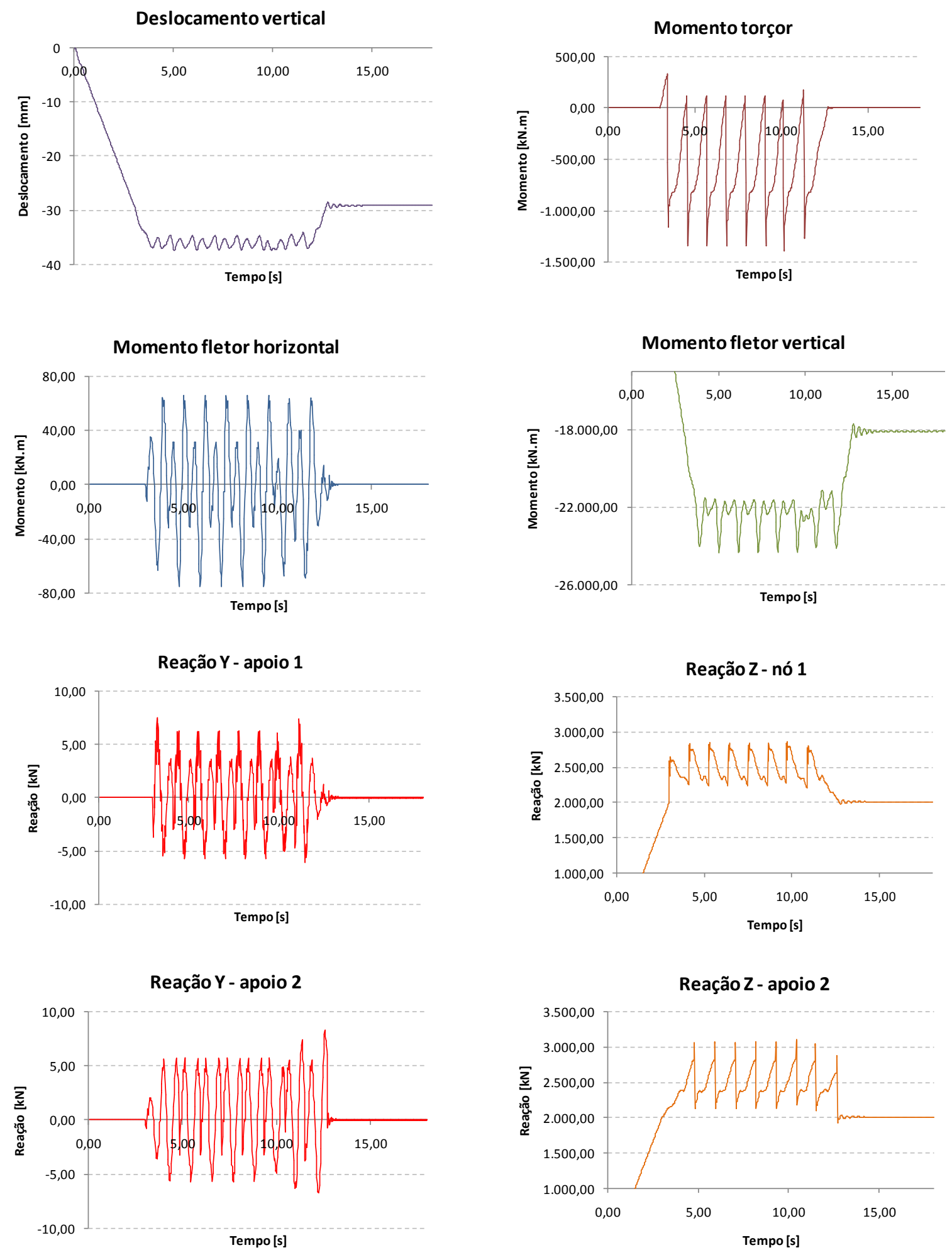

Figura 42: Resultados- análise dinâmica para $n=5$ 

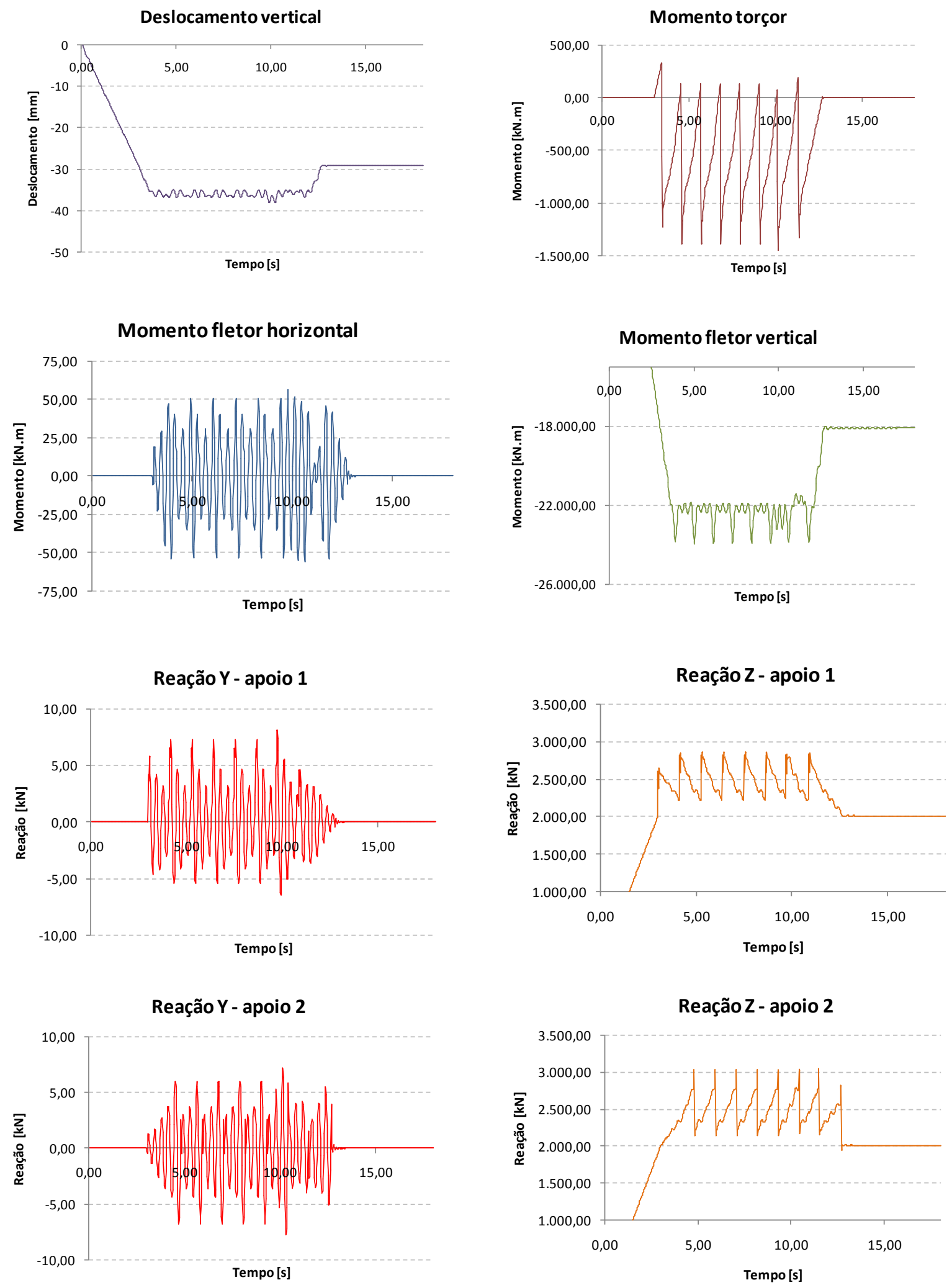

Figura 43: Resultados - análise dinâmica para $n=10$ 

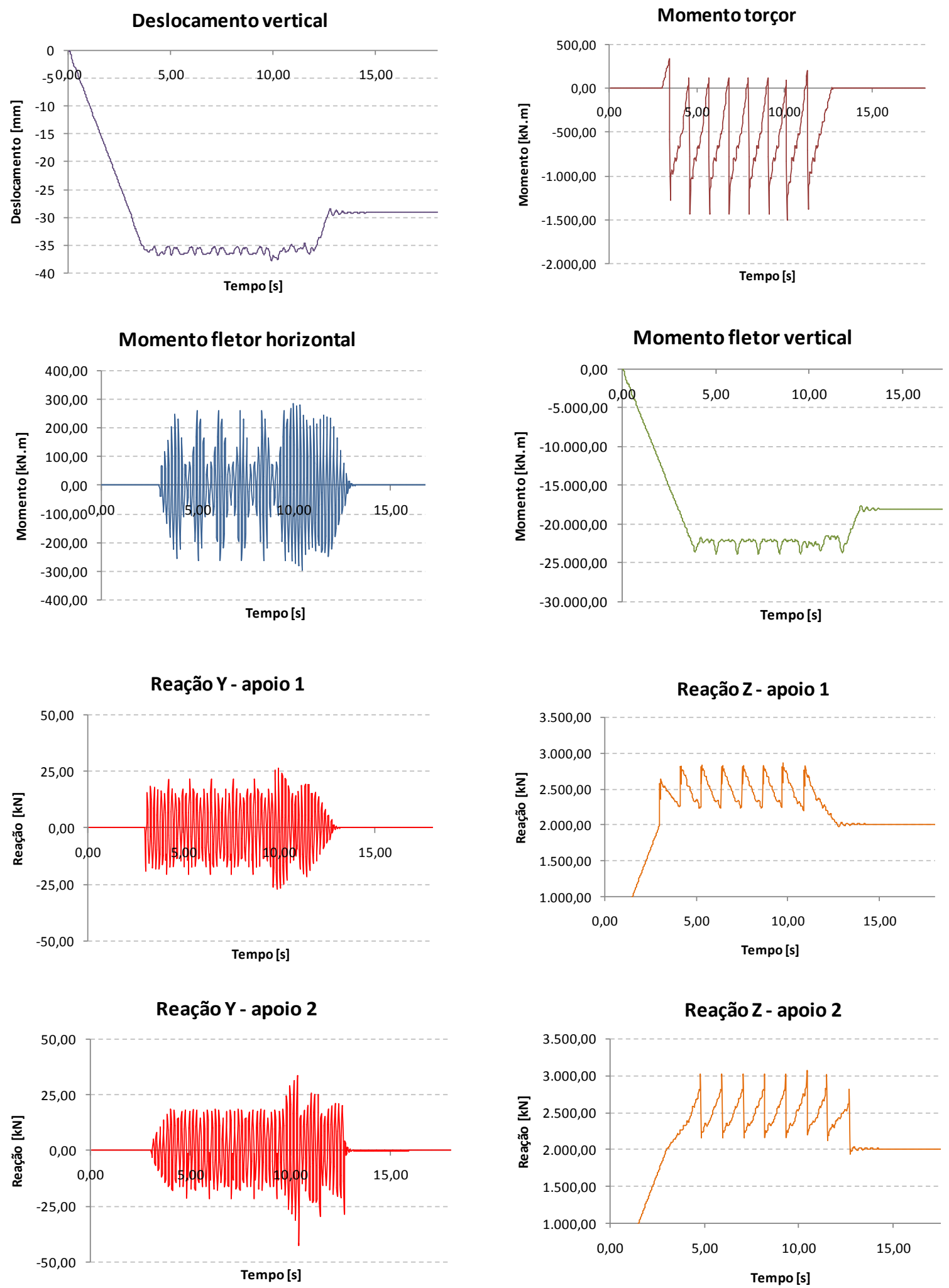

Figura 44: Resultados - análise dinâmica para $n=20$ 

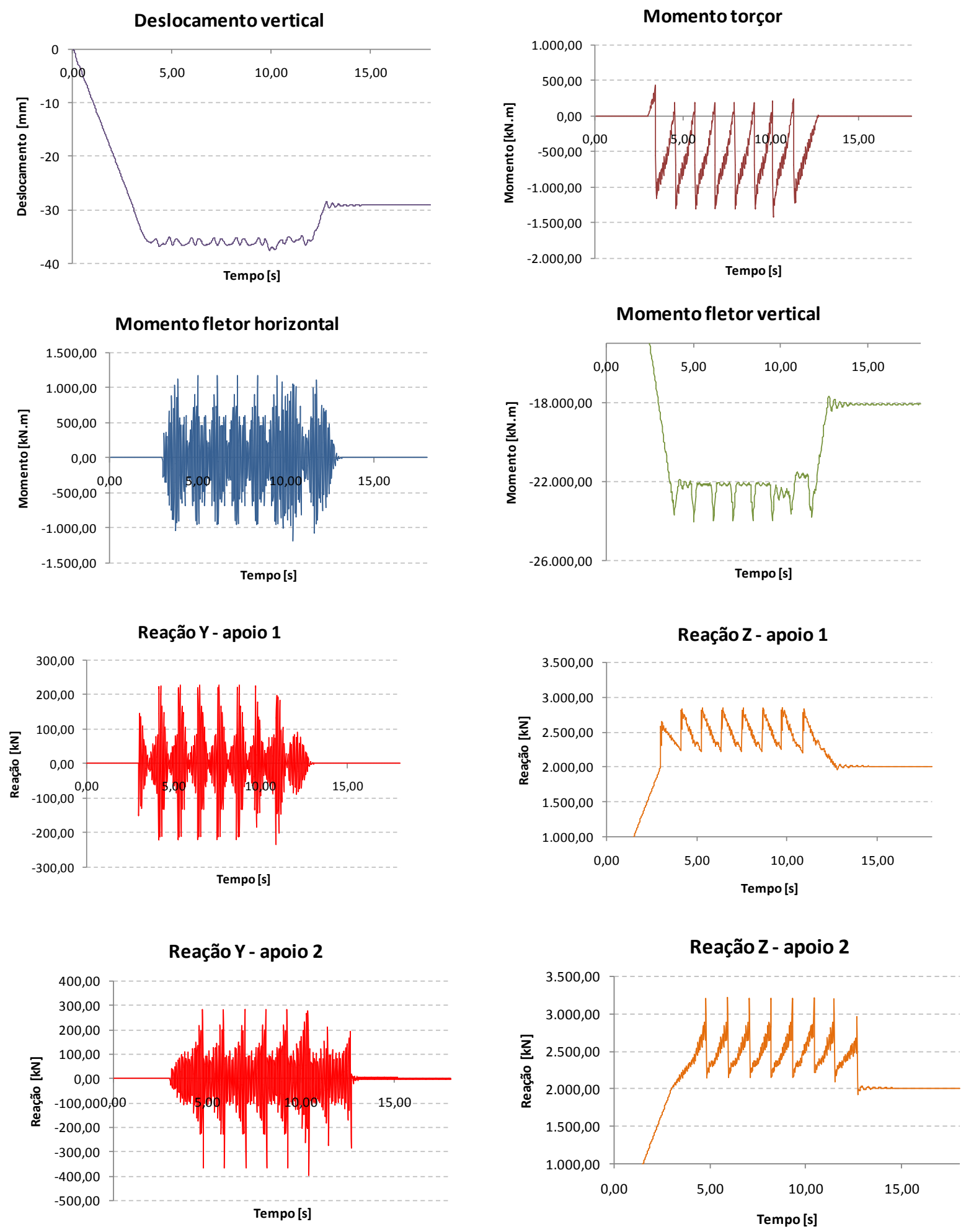

Figura 45: Resultados - análise dinâmica para $n=40$

A seguir, são apresentados os resultados obtidos para as análises dinâmicas descritas para o cenário considerando os esforços de interação em regime transiente. 

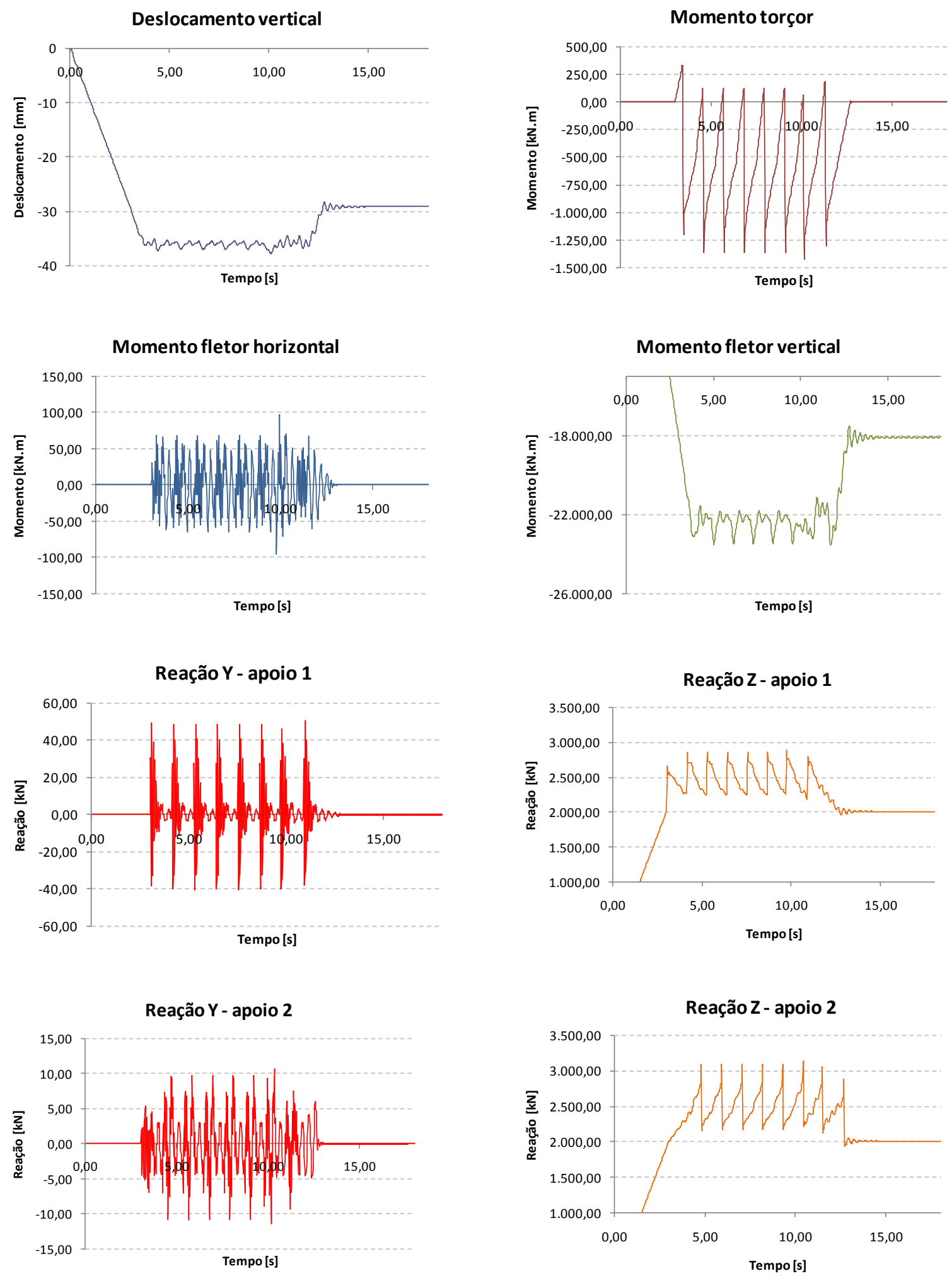

Figura 46: Resultados - análise dinâmica para $n=10$ 


\section{CONCLUSÕES}

Analisando-se primeiramente os modos de vibração encontrados para os modelos da ponte e do trem, vale comentar que as frequências dos cinco primeiros modos do carro $(0,645 \mathrm{a} 1,667 \mathrm{~Hz})$ são inferiores à do primeiro modo de vibração da ponte $(3,307 \mathrm{~Hz})$. Porém, existe uma proximidade entre os valores obtidos para os modos 2 e 3 da ponte e 6 a 9 do carro, modos 4 a 6 da ponte e 10 e 11 do carro e ainda, modos 13 a 15 da ponte e 12 e 13 do carro. A Tabela 5 apresenta esta comparação.

Tabela 5: Modos de vibração

\begin{tabular}{c|c|c}
\hline Modos & $\begin{array}{c}\text { Frequência } \\
\text { Ponte [Hz] }\end{array}$ & $\begin{array}{c}\text { Frequência } \\
\text { Veículo [Hz] }\end{array}$ \\
\hline $1^{\circ}$ & 3,307 & 0,645 \\
\hline $2^{\circ}$ & 10,46 & 1,215 \\
\hline $3^{\circ}$ & 10,52 & 1,230 \\
\hline $4^{\circ}$ & 14,97 & 1,334 \\
\hline $5^{\circ}$ & 16,30 & 1,667 \\
\hline $6^{\circ}$ & 16,73 & 9,044 \\
\hline $7^{\circ}$ & 17,41 & 9,055 \\
\hline $8^{\circ}$ & 17,67 & 11,720 \\
\hline $9^{\circ}$ & 18,16 & 11,720 \\
\hline $10^{\circ}$ & 18,48 & 15,480 \\
\hline $11^{\circ}$ & 18,68 & 15,480 \\
\hline $12^{\circ}$ & 20,00 & 21,960 \\
\hline $13^{\circ}$ & 21,60 & 21,960 \\
\hline $14^{\circ}$ & 21,97 & 24,990 \\
\hline $15^{\circ}$ & 22,62 & 24,990 \\
\hline
\end{tabular}

Com relação aos resultados de esforços, a Tabela 6 apresenta os valores máximos obtidos em cada situação analisada. Como se pode perceber nas análises dinâmicas cujos esforços estão em regime permanente, os resultados de momento fletor no plano horizontal e reação horizontal transversal nos apoios para os valores de $n$ iguais a 20 e 40 foram maiores que os decorrentes da análise quase-estática; isto também ocorreu para a reação vertical para $p$ caso de $n$ igual a 40; para o deslocamento vertical no meio do vão, momentos torçor e fletor no plano vertical, os resultados foram menores que os da análise quase-estática para todos os casos analisados. Para a análise com $\mathrm{n}$ igual a 10 considerando o regime transiente, a 
resposta para os esforços reações horizontal transversal e vertical também foram maiores que os da análise quase-estática.

Tabela 6: Valores máximos obtidos em cada análise [kN.m, kN e mm]

\begin{tabular}{|c|c|c|c|c|c|c|}
\hline \multirow{3}{*}{ Esforços } & \multirow{3}{*}{$\begin{array}{c}\text { Análise quase- } \\
\text { estática }\end{array}$} & \multicolumn{5}{|c|}{ Análise dinâmica } \\
\hline & & \multicolumn{4}{|c|}{ Regime permanente } & \multirow{2}{*}{$\begin{array}{c}\text { Com transiente } \\
\mathrm{n}=10\end{array}$} \\
\hline & & $n=5$ & $n=10$ & $\mathrm{n}=\mathbf{2 0}$ & $n=40$ & \\
\hline Momento torçor & $-1.677,05$ & $-1.385,71$ & $-1.442,18$ & $-1.502,49$ & $-1.416,79$ & $-1.421,97$ \\
\hline Momento fletor horizontal & $-268,70$ & $-75,45$ & 56,25 & $-295,45$ & $-1.190,64$ & 96,08 \\
\hline Momento fletor vertical & $-25.196,40$ & $-24.373,80$ & $-23.961,20$ & $-23.892,90$ & $-24.047,00$ & $-23.554,80$ \\
\hline Deslocamento vertical & $-38,35$ & $-37,44$ & $-38,14$ & $-37,83$ & $-37,65$ & $-37,82$ \\
\hline Reação Y - apoio 1 & $-41,05$ & 7,44 & 8,10 & $-27,08$ & $-236,06$ & 50,56 \\
\hline Reação Y - apoio 2 & $-41,05$ & 8,28 & $-7,79$ & $-41,42$ & $-396,81$ & $-11,44$ \\
\hline Reação Z - apoio 1 & $3.095,13$ & $2.855,43$ & $2.854,16$ & $2.853,62$ & $2.847,32$ & $2.881,87$ \\
\hline Reação Z - apoio 2 & $3.095,13$ & $3.088,02$ & $3.028,29$ & $3.055,11$ & $3.198,95$ & $3.124,88$ \\
\hline
\end{tabular}

O acréscimo de esforço real referente ao coeficiente de impacto no caso da análise quase-estática e à análise dinâmica para os esforços de momento fletor no plano vertical, deslocamento vertical e reação vertical no apoio foram obtidos subtraindo-se o efeito do peso próprio da estrutura do correspondente valor total máximo encontrado. A Tabela 7 apresenta essa diferença.

Tabela 7: Acréscimo da resposta referente ao coeficiente de impacto e análise dinâmica

\begin{tabular}{|c|c|c|c|c|c|c|}
\hline \multirow{3}{*}{ Esforços } & \multirow{3}{*}{$\begin{array}{c}\text { Análise quase- } \\
\text { estática }\end{array}$} & \multicolumn{5}{|c|}{ Análise dinâmica } \\
\hline & & \multicolumn{4}{|c|}{ Regime permanente } & \multirow{2}{*}{$\begin{array}{c}\text { Com transiente } \\
\mathrm{n}=10\end{array}$} \\
\hline & & $n=5$ & $n=10$ & $\mathrm{n}=\mathbf{2 0}$ & $n=40$ & \\
\hline My - My-pp [kN.m] & $-7.196,40$ & $-6.373,80$ & $-5.961,20$ & $-5.892,90$ & $-6.047,00$ & $-5.554,80$ \\
\hline$\Delta-\Delta p p[m m]$ & $-9,35$ & $-8,44$ & $-9,14$ & $-8,83$ & $-8,65$ & $-8,82$ \\
\hline Rz - Rz-pp apoio 1 [kN] & $1.095,13$ & 855,43 & 854,16 & 853,62 & 847,32 & 881,87 \\
\hline Rz - Rz-pp apoio 2 [kN] & $1.095,13$ & $1.088,02$ & $1.028,29$ & $1.055,11$ & $1.198,95$ & $1.124,88$ \\
\hline
\end{tabular}

Assim, é possível obter o valor do fator de amplificação dinâmica nos esforços apresentados na Tabela 7, para cada cenário analisado, conforme a Tabela 8.

Tabela 8: Fatores de amplificação dinâmica

\begin{tabular}{c|c|c|c|c|c|c}
\hline \multirow{2}{*}{ Esforços } & \multirow{2}{*}{$\begin{array}{c}\text { Análise } \\
\text { quase- }\end{array}$} & \multicolumn{7}{|c}{ Análise dinâmica } & Com transiente \\
\cline { 3 - 7 } & estática & $\mathbf{n = 5}$ & $\mathbf{n = 1 0}$ & $\mathbf{n = 2 0}$ & $\mathbf{n = 4 0}$ & $\mathbf{n = 1 0}$ \\
\cline { 3 - 7 } & 1,321 & 1,09 & 1,14 & 1,18 & 1,12 & 1,12 \\
\hline Momento torçor & 1,321 & 1,17 & 1,09 & 1,08 & 1,11 & 1,02 \\
\hline Momento fletor vertical & 1,321 & 1,19 & 1,29 & 1,25 & 1,22 & 1,25 \\
\hline Deslocamento vertical & 1,321 & 1,03 & 1,03 & 1,03 & 1,02 & 1,06 \\
\hline Reação Z - apoio 1 & 1,32 & 1,45 & 1,36 \\
\hline Reação Z - apoio 2 & 1,321 & 1,31 & 1,24 & 1,27 & &
\end{tabular}

De acordo com a Tabela 8, pode-se perceber que os esforços sofrem influência do valor utilizado para $\mathrm{n}$ e para a velocidade adotada, notando-se grande dispersão dos resultados. No caso dos esforços de momentos torçor e fletor no plano vertical e deslocamento vertical, a norma atende a todas as situações analisadas na Tabela 8, 
ou seja, o coeficiente prescrito por ela, de 1,321, neste estudo de caso, foi maior que os obtidos nas análises dinâmicas Para a reação vertical no apoio, os casos de $\mathrm{n}$ igual a 40, para o regime permanente, e o cenário analisado com transiente, apresentaram fatores de amplificação dinâmica equivalente à 1,45 e 1,36, respectivamente, e portanto, maiores aos da análise quase-estática. Entretanto, para todos os esforços comentados, podem existir outras combinações $(n, V)$ mais críticas do que as discutidas na Tabela 8, desde que a frequência dominante na força de interação $F_{Z}$ seja próxima de algum modo de flexão no plano vertical.

Já para os esforços de momento fletor no plano horizontal e reação horizontal transversal, ao invés de determinar o coeficiente de amplificação dinâmica, pela indisponibilidade do valor de referência estático, obteve-se apenas a relação entre os resultados obtidos nas análises dinâmicas e na análise quase-estática prescrita pela norma. A Tabela 9 apresenta essas relações.

Tabela 9: Relação entre esforços da análise dinâmica e os da análise quase-estática

\begin{tabular}{|c|c|c|c|c|c|}
\hline \multirow{3}{*}{ Esforços } & \multicolumn{5}{|c|}{ Análise dinâmica } \\
\hline & \multicolumn{4}{|c|}{ Regime permanente } & \multirow{2}{*}{$\begin{array}{c}\text { Com transiente } \\
n=10\end{array}$} \\
\hline & $n=5$ & $\mathrm{n}=10$ & $n=20$ & $\mathrm{n}=\mathbf{4 0}$ & \\
\hline Momento fletor horizontal & 0,28 & 0,21 & 1,10 & 4,43 & 0,36 \\
\hline Reação Y - apoio 1 & 0,18 & 0,20 & 0,66 & 5,75 & 1,23 \\
\hline Reação Y - apoio 2 & 0,20 & 0,19 & 1,01 & 9,67 & 0,28 \\
\hline
\end{tabular}

De acordo com a Tabela 9, percebe-se uma enorme variação de valores em função do cenário analisado para os dois esforços. Porém, para n igual a 20 e 40, houve um aumento muito expressivo nos esforços. Reitera-se que, apesar de se tratar aparentemente de situações extremas e, eventualmente, pouco realistas, o caso do cenário com $\mathrm{n}$ igual a 20 pode ser interpretado como sendo equivalente ao de $\mathrm{n}$ igual a $10 \mathrm{com}$ velocidade de $40 \mathrm{~m} / \mathrm{s}(144 \mathrm{~km} / \mathrm{h})$, ou ainda ao de $\mathrm{n}$ igual a $5 \mathrm{com}$ velocidade de $80 \mathrm{~m} / \mathrm{s}(288 \mathrm{~km} / \mathrm{h})$; da mesma forma, $\mathrm{n}$ igual a 40 pode ser interpretado como equivalente ao de $n$ igual a $20 \mathrm{com}$ velocidade de $40 \mathrm{~m} / \mathrm{s}$, ou $\mathrm{n}$ igual a $10 \mathrm{com}$ velocidade de $80 \mathrm{~m} / \mathrm{s}$. Com relação às frequências dominantes dos esforços de interação trem-trilho, a Tabela 10 apresenta os valores obtidos para cada esforço em cada cenário analisado. 
Tabela 10: Frequências dos esforços de interação [Hz]

\begin{tabular}{c|c|c|c|c}
\cline { 2 - 5 } & $\mathbf{n = 5}$ & $\mathbf{n = 1 0}$ & $\mathbf{n = 2 0}$ & $\mathbf{n = 4 0}$ \\
\hline $\mathrm{Fy}$ & 1,39 & 2,86 & 5,56 & 14,30 \\
\hline $\mathrm{Fz}$ & 1,33 & 2,86 & 5,56 & 12,50 \\
\hline $\mathrm{Mx}$ & 1,33 & 2,86 & 5,56 & 14,30 \\
\hline $\mathrm{My}$ & 1,33 & 2,70 & 6,67 & 12,50 \\
\hline $\mathrm{Mz}$ & 1,33 & 2,70 & 5,88 & 10,00 \\
\hline
\end{tabular}

Conforme apresentado na Tabela 10, as frequências obtidas para os esforços de interação para o cenário com valor de $\mathrm{n}$ igual a 5 são próximas à frequência referente ao quarto modo de vibração do carro $(f=1,33 \mathrm{~Hz})$, modo de bounce, colocando os esforços de interação em ressonância com o ele. Neste estudo de caso, percebeu-se que os esforços obtidos para o cenário com $n$ igual a 5 , apesar de terem pequena amplitude, produziram respostas significativas por conta da ressonância. Quanto ao cenário de $\mathrm{n}=40$ e velocidade de $20 \mathrm{~m} / \mathrm{s}$ (ou os casos equivalentes, já mencionados), nota-se que produziu momento de interação $M_{Z}$ com frequência dominante da ordem de $10 \mathrm{~Hz}$, portanto quase-ressonante com o segundo e terceiro modos do tabuleiro da ponte, amplificando consideravelmente os esforços no plano horizontal, conforme já notado. Por outro lado, a força de interação $F_{Z}$ apresentou para $\mathrm{n}=40$ frequência da ordem de $12,5 \mathrm{~Hz}$, portanto não-ressonante em relação aos mesmos modos, não amplificando na mesma medida a flexão no plano vertical, nem a torção. Os demais cenários, apesar de não estarem em ressonância, foram relevantes também em função do aumento das intensidades dos esforços de interação. Reitera-se que, em princípio, pode existir uma combinação $(n, V)$ que leve a esforços de interação ressonantes, seja com o carro, seja com o tabuleiro da ponte, conduzindo, eventualmente, a grandes amplificações da resposta dinâmica, cenários que o procedimento quase-estático proposto pela norma não permite antecipar.

Vale comentar ainda que se adotou para as análises dinâmicas uma taxa de amortecimento relativamente alta $(5 \%)$ para todos os modos de vibração. Assim, especialmente nas situações de proximidade com ressonâncias, é de se esperar que amplificações dinâmicas ainda maiores possam ocorrer, caso valores mais baixos e, eventualmente mais realistas da taxa de amortecimento sejam utilizados.

Por fim, observa-se que nesta metodologia considerou-se 0 pavimento indeslocável. Obviamente, assumir esta condição leva a esforços de interação superestimados, porém este problema pode ser contornado fazendo-se a correção 
dos deslocamentos impostos na interface trilho-roda, tanto no plano vertical quanto no plano horizontal, por meio de um procedimento iterativo, conforme já adotado em Moroz (2009), de forma que se somem os deslocamentos do tabuleiro obtidos para a primeira determinação dos esforços de interação (com pavimento indeslocável) à excitação de suporte aplicada nas rodas do trem para simular a presença das irregularidades. Com isso, pode-se obter os novos esforços de interação, que serão reaplicados ao modelo unifilar do tabuleiro para determinar os novos deslocamentos, repetindo-se o procedimento até que resultados sejam muito próximos aos do passo anterior. Este procedimento levaria a uma redução nos resultados finais. 


\section{SUGESTÕES PARA TRABALHOS FUTUROS}

A realização deste trabalho mostrou que a análise dinâmica é influenciada por muitos fatores, como a função adotada para representar as irregularidades, velocidade e parâmetros do carro, geometria e vinculação da ponte, sistemas estruturais empregados, entre outros.

Assim, seria interessante aplicar esta metodologia simplificada de análise dinâmica a pontes com mesmo comprimento de vão (e, portanto, mesmo coeficiente de impacto prescrito pela norma NBR 7187), mas com seções transversais distintas (em caixão, com paredes mais esbeltas), ou ainda com outros sistemas estruturais (como ponte em viga contínua celular, ponte em grelha, ponte pênsil, ponte estaiada).

Não se pretende, com um único estudo de caso, como o apresentado nesta dissertação, fazer um julgamento definitivo das disposições normativas que regem o projeto de pontes ferroviárias em nosso País. Entretanto, após um amplo conjunto de análises paramétricas, para cada tipo de sistema estrutural de ponte ferroviária, poderse-á, efetivamente, chegar a critérios que se baseiem no desempenho estrutural perante as cargas móveis decorrentes da passagem de trens, com melhor avaliação quantitativa e qualitativa da segurança da estrutura com relação a estados limites últimos ou de serviço, inclusive com análise de fadiga dos componentes estruturais e estimativa da sua vida útil.

No caso de pontes suspensas, seria interessante analisar o efeito dinâmico combinado do vento e das cargas móveis decorrentes da passagem do trem. 


\section{REFERÊNCIAS BIBLIOGRÁFICAS}

[1] ASSOCIAÇÃO BRASILEIRA DE NORMAS TÉCNICAS, NBR 7189 - Cargas Móveis para Projeto Estrutural - 1985. 12

[2] ASSOCIAÇÃO BRASILEIRA DE NORMAS TÉCNICAS, NBR 7187 - Projeto de Pontes de Concreto Armado e de Concreto Protendido - Procedimento - 2003. 13

[3] BLEJWAS, T. E., FENG, C. C. and AYRE, R. S., 1979, Dynamic Interaction of Moving Vehicles and Structures. Journal of Sound and Vibration, 67, 513-521.

[4] CLEMENTE, L. G., BORGES L. A. e STUCCHI, F., 1989. Projeto de viaduto unicelular para pista dupla na extensão da linha Norte/Sul do Metrô de São Paulo - Anais do Primeiro Simpósio EPUSP de Estrutura de Concreto

[5] CHEN, Y. and LI, C. Y., 2000, Dynamic Response of Elevated High-Speed Railway. Journal of Bridge Engineering, Vol. 5, n², 124-130.

[6] Companhia Brasileira de Trens Urbanos - CBTU. Ficha Técnica ㄲo 2410.

[7] Companhia Brasileira de Trens Urbanos - CBTU. Ficha Técnica oㅡ 2411.

[8] CORREA, W. L., 2008, Controle das Vibrações Induzidas pela Interação Dinâmica entre Trens-Trilhos-Dormentes-Estrutura de Aço de Pontes Ferroviárias. Tese, COPPE/UFRJ, Rio de Janeiro, RJ, Brasil.

[9] Eurocode EN 1991-2.

[10] FRÝBA, L., 1980, Estimation of Fatigue Life of Railway Bridges under Traffic Loads. Journal of Sound and Vibration, 70, 527-541.

[11] GARINEI, A. and RISITANO, G., 2007. Vibrations of Railway Bridges for High Speed Trains under Moving Loads Varying in Time. Engineering Structures, 30, 724-732.

[12] LIN, Y. H. and TRETHEWEY, M. W., 1990. Finite Element Analysis of Elastic Beams Subjected to Moving Dynamic Loads. Journal of Sound and Vibration, 136, 323-342.

[13] LIU, K., ROECK, G. and LOMBAERT, G., 2009, The Effect of Dynamic TrainBridge Interaction on the Bridge Response during a Train Passage. Journal of Sound and Vibration, 325, 240-251.

[14] MAJKA, M. and HARTNETT, M., 2009. Dynamic Response of Bridges to Moving Trains: A Study on Effects of Random Track Irregularities and Bridge Skewness. Journal Computers \& Structures, 87, 1233-1252.

[15] MOROZ, F. V., 2009, Uma Metodologia para a Análise da Influência no Tráfego de Carros Pesados na Resposta Dinâmica de Pontes Rodoviárias. Dissertação de Mestrado, POLI/USP, São Paulo, SP, Brasil.

[16] OLSSON, M., 1985. Finite Element, Modal Co-Ordinate Analysis of Structures Subjected to Moving Loads. Journal of Sound and Vibration, 99, 1-12. 
[17] WILLIS, R. 1849. Appendix to the Report of Commissioners Appointed to Inquire into the Application of Iron to Railway Structures. Stationary Office, London.

[18] XIA, H., ZANG, N. and GAO, R., 2005, Experimental analysis of railway bridge under high-speed trains. Journal of Sound and Vibration 282, 517-528.

[19] XIA, H., XU, Y. L. and CHAN, T. H. T., 2000, Dynamic Interaction of Long Suspension Bridges with Running Trains. Journal of Sound and Vibration 237, 263-280.

[20] XU, Y. L., XIA, H. and YAN, Q. S., 2003, Dynamic Response of Suspension Bridge to High Wind and Running Train. Journal of Bridge Engineering, Vol. 8, $\mathrm{n}^{\circ} 1,46-55$.

[21] ZHANG, Q. L., VROUWENVELDER, A. and WARDENIER. J., 2000. Numerical Simulation of Train-Bridge Interactive Dynamics. Journal Computers \& Structures, 79, 1059-1075.

[22] http://www.antf.org.br

[23] www.cptm.sp.gov.br

[24] http://vfco.brazilia.jor.br/ferrovias/ef/55engates.htm

[25] http://www.guiadotrc.com.br/infra/vagoes.asp

[26] http://www.flickr.com/photos/vagner66-trens

[27] http://www.portogente.com.br/texto.php?cod=11670\&sec=113

[28] http://www.ime.eb.br/ webde2/prof/ethomaz/pontes/ime protendido 02 ponte ferroviaria pontes.pdf

[29] http://www.novomilenio.inf.br/santos/h0102n.htm 